\title{
Silicon Acetal Metathesis Polymerization (SAMP)
}

Ertugrul Sahmetlioglu, ${ }^{\S}$ Ha Thi Hoang Nguyen, Olivier Nsengiyumva, Ersen Göktürk,,${ }^{\natural}$ and Stephen A. Miller*

The George and Josephine Butler Laboratory for Polymer Research, Department of Chemistry, University of Florida Gainesville, Florida 32611-7200, USA

$\S$ Present address: Erciyes University, Nanotechnology Research Center (ERNAM) Kayseri, 38039, Turkey

"Present address: Mustafa Kemal University, Department of Chemistry, 31001, Antakya/Hatay, Turkey

\section{Supporting Information}

Supporting Information Available: Synthetic details, complete polymer characterization data, and kinetic studies.

\section{Table of Contents}

General Considerations and Instrumentation

Polymerizations

Summary of Polymerization Data

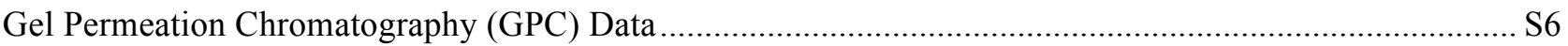

Differential Scanning Calorimetry (DSC) Thermograms .......................................................................... S14

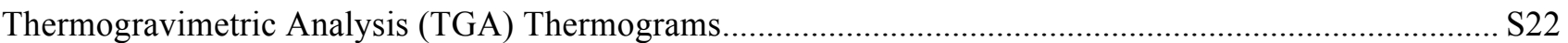

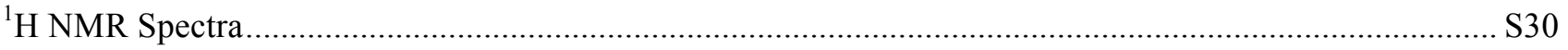

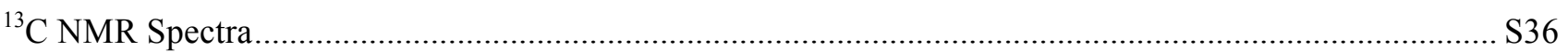

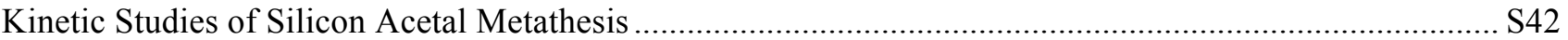

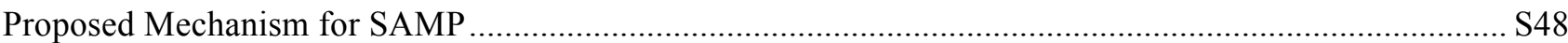

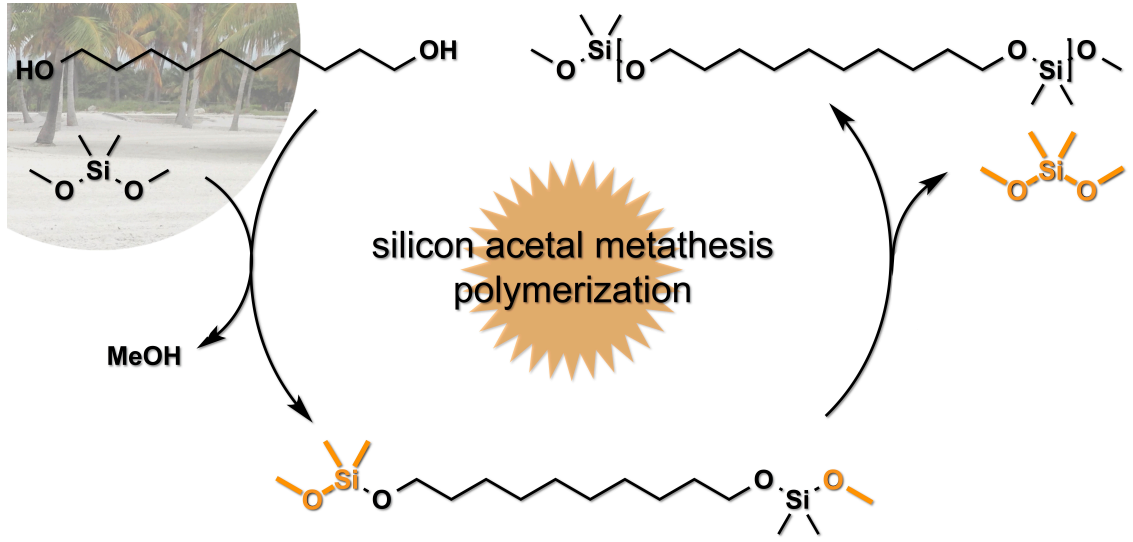




\section{General Considerations and Instrumentation Materials}

1,6-hexanediol (97\%), 1,8-octanediol (99\%), and 1,9nonanediol (99\%) were purchased from Acros Organics and were further purified by recrystallization from ethyl acetate before use. 1,10-decanediol (99\%) was purchased from Acros Organics and was purified by recrystallization from 1,2-dichloroethane. (+)-cis-1,2,2-trimethylcyclopentane-1,3-dimethanol (TCDM) which was obtained from Dr. Feng Liu from Nanchang University, Nanchang, China, was used as received. Hydroquinone bis(2hydroxyethyl) ether (99\%) was purchased from Acros Organics and was used as received. Bis(2-hydroxyethyl) terephthalate, Diethoxydimethylsilane (97\%) and dimethoxydimethylsilane (98\%) were purchased from Sigma Aldrich and used as received. Dimethylmethoxychlorosilane (85\%) was purchased from Gelest, Inc. and was used as received. Methanesulfonic acid (99.5\%) was purchased from Sigma Aldrich and was used as received. Chloroform for polymerizations was dried using an MBraun Solvent Purification System (MB SPS). The catalysts, including para-toluenesulfonic acid ( $p$-TSA) monohydrate and triflic acid were purchased from Sigma Aldrich and were used as received. NMR solvents, including deuterated chloroform $\left(\mathrm{CDCl}_{3}\right)$ and deuterated benzene (benzene- $d_{6}$ ), which do not contain tetramethylsilane (TMS), were purchased from Cambridge Isotope Laboratories. All other chemicals, unless expressly mentioned, were utilized as received.

\section{Characterizations}

Proton and carbon nuclear magnetic resonance $\left({ }^{1} \mathrm{H}\right.$ and ${ }^{13} \mathrm{C}$ NMR) spectra were recorded using an Inova 500 $\mathrm{MHz}$ spectrometer. Chemical shifts are reported in parts per million (ppm) relative to the specified NMR solvent signal. Coupling constants $(J)$ are reported in Hertz $(\mathrm{Hz})$. Multiplicities are reported using the following abbreviations: s, singlet; d, doublet; t, triplet; q, quartet; quin, quintuplet; m, multiplet; br, broad.

Differential scanning calorimetry (DSC) thermograms were obtained with a DSC Q1000 from TA instruments. About 1.5-3 mg of each sample were massed and added to a sealed pan that passed through a heat/cool/heat cycle at $10{ }^{\circ} \mathrm{C} / \mathrm{min}$. Reported data are from the second full cycle. The temperature ranged from about -80 to $200^{\circ} \mathrm{C}$, depending on the samples.

Thermogravimetric analyses (TGA) were measured under nitrogen with a TGA Q5000 from TA Instruments. About 5-10 mg of each sample were heated at $20^{\circ} \mathrm{C} / \mathrm{min}$ from 25 to $600^{\circ} \mathrm{C}$.

Gel Permeation Chromatography (GPC) analysis (Entries 1-12 in Table S1) was carried out using a Perkin Elmer Series 200 apparatus with a refractive index (RI) detector under the following conditions: TSK gel AM SEC Gel column and dimethylformamide (DMF) eluent at a flow rate of $1.0 \mathrm{~mL} / \mathrm{min}$. The calibration curves for GPC analysis were obtained using polystyrene (PS) standards.
Additionally, gel permeation chromatography (GPC) was performed (Entries 13-16 in Table S1) using an Agilent 1100 series liquid chromatography system with an internal differential refractive index detector. The columns used were two Waters Styragel HR 4E tetrahydrofuran (THF) columns (10 micromPD, $7.8 \mathrm{~mm}$ i.d., $300 \mathrm{~mm}$ length). The mobile phase is HPLC grade tetrahydrofuran (THF) with a flow rate of $1.0 \mathrm{~mL} / \mathrm{min}$. Calibration was performed using a 10 point calibration with narrow polydispersity polystyrene (PS) standards.

\section{Polymerizations \\ Polymerization apparatus}

The polymerizations were typically conducted in a round bottom flask, connected to a rotary evaporation bump trap, connected to a vacuum line. With this apparatus, the by-product of condensation could be collected and seen in the bump trap. At the same time, all volatiles could be removed without changing the initial glassware configuration.

\section{General work-up procedure for polymerization entries 1-12 in Table S1}

After cooling to room temperature, the mixture was poured into cold, basic ethanol/methanol to terminate the polymerization. The system was filtered and the collected polymer was washed with copious amounts of methanol, and diethyl ether. The polymer was dried under vacuum overnight.

\section{General work-up procedure for polymerization entries 13-16 in Table S1}

The polymers were melted by a hand-held heat gun and removed from the round bottom flask. They were used without any further purification.

\section{Representative Polymerization procedure for entries 1-4 in Table $S 1$}

Equivalent molar amounts of dichlorodimethylsilane and an alkylenediol were stirred under a nitrogen atmosphere for $24 \mathrm{~h}$ at $60{ }^{\circ} \mathrm{C}$. After $24 \mathrm{~h}$, the system was placed under dynamic vacuum to increase the degree of polymerization by removal of the condensation product for 2.5 hours. The reaction temperature was then raised to $75^{\circ} \mathrm{C}$ under vacuum for an additional 3.5 hours.

\section{Polyhexylene silicon acetal}

$-\left[\mathrm{OSiMe}_{2} \mathrm{O}\left(\mathrm{CH}_{2}\right)_{6}\right]-$

Table S1, Entry 1. ${ }^{1} \mathrm{H}$ NMR $\left(\mathrm{CDCl}_{3}\right): \delta \mathrm{ppm}-0.05-0.19$ (m, $6 \mathrm{H}), 1.15-1.43(\mathrm{~m}, 4 \mathrm{H}), 1.43-1.77$ (m, $4 \mathrm{H}), 3.41$ - $3.68(\mathrm{~m}, 4 \mathrm{H}) .{ }^{13} \mathrm{C} \mathrm{NMR}\left(\mathrm{CDCl}_{3}\right): \delta \mathrm{ppm}-3.4,25.5$, 32.4, 62.3 .

\section{Polyoctylene silicon acetal \\ $-\left[\mathrm{OSiMe}_{2} \mathrm{O}\left(\mathrm{CH}_{2}\right)_{8}\right]-$}

Table S1, Entry 2. ${ }^{1} \mathrm{H}$ NMR $\left(\mathrm{CDCl}_{3}\right): \delta \mathrm{ppm}-0.11-0.23$ (m, $6 \mathrm{H}), 1.27$ (br. s, $8 \mathrm{H}), 1.43-1.63$ (m, $4 \mathrm{H}), 3.34-$ $3.70(\mathrm{~m}, 4 \mathrm{H}) .{ }^{13} \mathrm{C} \mathrm{NMR}\left(\mathrm{CDCl}_{3}\right): \delta \mathrm{ppm}-3.3,25.7,29.3$, $32.5,62.4$. 
Polynonylene silicon acetal

$-\left[\mathrm{OSiMe}_{2} \mathrm{O}\left(\mathrm{CH}_{2}\right)_{9}\right]-$

Table S1, Entry 3. ${ }^{1} \mathrm{H}$ NMR $\left(\mathrm{CDCl}_{3}\right)$ : $\delta$ ppm $-0.09-0.24$

$(\mathrm{m}, 6 \mathrm{H}), 1.26$ (br. s, $10 \mathrm{H}), 1.44-1.60$ (m, $4 \mathrm{H}), 3.48-$

$3.72(\mathrm{~m}, 4 \mathrm{H}) .{ }^{13} \mathrm{C} \mathrm{NMR}\left(\mathrm{CDCl}_{3}\right): \delta \mathrm{ppm}-3.3,25.7,29.3$, $29.5,32.5,62.4$.

\section{Polydecylene silicon acetal}

$-\left[\mathrm{OSiMe}_{2} \mathrm{O}\left(\mathrm{CH}_{2}\right)_{10}\right]-$

Table S1, Entry 4. ${ }^{1} \mathrm{H}$ NMR $\left(\mathrm{CDCl}_{3}\right): \delta$ ppm $-0.09-0.32$ (m, $6 \mathrm{H}), 1.28$ (br. s, $12 \mathrm{H}), 1.46-1.64$ (m, $4 \mathrm{H}), 3.53-$ $3.72(\mathrm{~m}, 4 \mathrm{H}) .{ }^{13} \mathrm{C} \mathrm{NMR}\left(\mathrm{CDCl}_{3}\right): \delta \mathrm{ppm}-3.2,25.7,29.4$, $29.5,32.7,62.9$.

\section{Representative Polymerization procedure for entries} 5-12 in Table $S 1$

Equimolar amounts of dimethoxydimethylsilane, an alkyldiol, and $1 \mathrm{~mol} \%$ (vs. monomers) of a Brønsted acid catalyst ( $p$-TSA for entries 5-8 or triflic acid for entries $9-12$ ) in $1.5 \mathrm{~mL}$ chloroform were stirred under a nitrogen atmosphere for $24 \mathrm{~h}$ at $60{ }^{\circ} \mathrm{C}$. After $24 \mathrm{~h}$, the system was placed under dynamic vacuum to increase the degree of polymerization by removal of the condensation product for 2.5 hours. The reaction temperature was then raised to $75^{\circ} \mathrm{C}$ under vacuum for an additional 3.5 hours.

\section{Polyhexylene silicon acetal}

$-\left[\mathrm{OSiMe}_{2} \mathrm{O}\left(\mathrm{CH}_{2}\right)_{6}\right]-$

Table S1, Entry 5. ${ }^{1} \mathrm{H}$ NMR $\left(\mathrm{CDCl}_{3}\right)$ : $\delta$ ppm $0.01-0.22$ (m, $6 \mathrm{H}), 1.21-1.47(\mathrm{~m}, 4 \mathrm{H}), 1.48-1.69(\mathrm{~m}, 4 \mathrm{H}), 3.63$ $(\mathrm{t}, J=6.6 \mathrm{~Hz}, 4 \mathrm{H}) .{ }^{13} \mathrm{C} \mathrm{NMR}\left(\mathrm{CDCl}_{3}\right): \delta \mathrm{ppm}-3.5,25.5$, $32.5,62.7$.

\section{Polyoctylene silicon acetal}

$-\left[\mathrm{OSiMe}_{2} \mathrm{O}\left(\mathrm{CH}_{2}\right)_{8}\right]-$

Table S1, Entry 6. ${ }^{1} \mathrm{H}$ NMR $\left(\mathrm{CDCl}_{3}\right)$ : $\delta \mathrm{ppm}-0.11-$ $0.25(\mathrm{~m}, 6 \mathrm{H}), 1.15-1.43(\mathrm{~m}, 8 \mathrm{H}), 1.47-1.65(\mathrm{~m}, 4 \mathrm{H})$, $3.53-3.75(\mathrm{~m}, 4 \mathrm{H}) .{ }^{13} \mathrm{C} \mathrm{NMR}\left(\mathrm{CDCl}_{3}\right): \delta \mathrm{ppm}-3.2$, 25.6, 29.3, 32.6, 62.9 .

\section{Polynonylene silicon acetal}

$-\left[\mathrm{OSiMe}_{2} \mathrm{O}\left(\mathrm{CH}_{2}\right)_{9}\right]-$

Table S1, Entry 7. ${ }^{1} \mathrm{H} \mathrm{NMR}\left(\mathrm{CDCl}_{3}\right): \delta \mathrm{ppm}-0.15-0.26$ $(\mathrm{m}, 6 \mathrm{H}), 1.28$ (br. s, $10 \mathrm{H}), 1.40-1.72(\mathrm{~m}, 4 \mathrm{H}), 3.29-$ $3.81(\mathrm{~m}, 4 \mathrm{H}) .{ }^{13} \mathrm{C} \mathrm{NMR}\left(\mathrm{CDCl}_{3}\right): \delta \mathrm{ppm}-3.3,25.6,29.2$, $29.4,32.5,62.8$.

\section{Polydecylene silicon acetal}

$-\left[\mathrm{OSiMe}_{2} \mathrm{O}\left(\mathrm{CH}_{2}\right)_{10}\right]-$

Table S1, Entry 8. ${ }^{1} \mathrm{H}$ NMR $\left(\mathrm{CDCl}_{3}\right)$ : $\delta$ ppm $-0.06-0.26$ $(\mathrm{m}, 6 \mathrm{H}), 1.27$ (br. s, $12 \mathrm{H}), 1.44-1.68$ (m, 4H), $3.45-$ $3.76(\mathrm{~m}, 4 \mathrm{H}) .{ }^{13} \mathrm{C} \mathrm{NMR}\left(\mathrm{CDCl}_{3}\right): \delta \mathrm{ppm}-3.3,25.7,29.4$, $29.5,32.5,62.5$.

\section{Polyhexylene silicon acetal}

$-\left[\mathrm{OSiMe}{ }_{2} \mathrm{O}\left(\mathrm{CH}_{2}\right)_{6}\right]-$

Table S1, Entry 9. ${ }^{1} \mathrm{H}$ NMR $\left(\mathrm{CDCl}_{3}\right): \delta \mathrm{ppm}-0.01$ (s, 6 H), 1.24 (br. s, 4 H), 1.44 (br. s, 4 H), 3.16 - 3.80 (m, 4 H). ${ }^{13} \mathrm{C} \mathrm{NMR}\left(\mathrm{CDCl}_{3}\right): \delta \mathrm{ppm}-3.5,25.3,32.4,62.2$.
Polyoctylene silicon acetal

$-\left[\mathrm{OSiMe}_{2} \mathrm{O}\left(\mathrm{CH}_{2}\right)_{8}\right]-$

Table S1, Entry 10. ${ }^{1} \mathrm{H}$ NMR $\left(\mathrm{CDCl}_{3}\right): \delta \mathrm{ppm}-0.12-$ 0.20 (m, $6 \mathrm{H}), 1.26$ (br. s, $8 \mathrm{H}), 1.41-1.64$ (m, $4 \mathrm{H}), 3.26$ - $3.73(\mathrm{~m}, 4 \mathrm{H}) .{ }^{13} \mathrm{C} \mathrm{NMR}\left(\mathrm{CDCl}_{3}\right): \delta \mathrm{ppm}-3.3,25.6$, $29.3,32.4,62.4$.

\section{Polynonylene silicon acetal}

$-\left[\mathrm{OSiMe}_{2} \mathrm{O}\left(\mathrm{CH}_{2}\right)_{9}\right]-$

Table S1, Entry 11. ${ }^{1} \mathrm{H} \mathrm{NMR}\left(\mathrm{CDCl}_{3}\right): \delta \mathrm{ppm}-0.14-$ $0.26(\mathrm{~m}, 6 \mathrm{H}), 1.26$ (br. s, $10 \mathrm{H}), 1.42-1.69(\mathrm{~m}, 4 \mathrm{H})$, $3.50-3.77(\mathrm{~m}, 4 \mathrm{H}) \cdot{ }^{13} \mathrm{C} \mathrm{NMR}\left(\mathrm{CDCl}_{3}\right): \delta \mathrm{ppm}-3.3$, 25.7, 29.3, 29.4, 32.5, 62.4.

\section{Polydecylene silicon acetal}

$-\left[\mathrm{OSiMe}_{2} \mathrm{O}\left(\mathrm{CH}_{2}\right)_{10}\right]-$

Table S1, Entry 12. ${ }^{1} \mathrm{H} \mathrm{NMR}\left(\mathrm{CDCl}_{3}\right): \delta \mathrm{ppm}-0.08-$ $0.27(\mathrm{~m}, 6 \mathrm{H}), 1.11-1.38(\mathrm{~m}, 12 \mathrm{H}), 1.41-1.66(\mathrm{~m}, 4$ $\mathrm{H}), 3.41-3.77(\mathrm{~m}, 4 \mathrm{H}) .{ }^{13} \mathrm{C} \mathrm{NMR}\left(\mathrm{CDCl}_{3}\right): \delta \mathrm{ppm}-3.3$, $25.7,29.3,29.5,32.5,62.4$.

Polymerization procedures for entries 13-16 in Table S1

\section{Polycamphoric silicon acetal}<smiles>CCOCC1(C)CCC(CO[Si](C)(C)C)C1(C)C</smiles>

Table S1, Entry 13. In a dry $50 \mathrm{~mL}$ round bottom flask, $0.50 \mathrm{~g} \quad(2.90 \mathrm{mmol})$ of $(+)-$ cis-1,2,2trimethylcyclopentane-1,3-dimethanol (TCDM), $0.70 \mathrm{~mL}$ $(4.06 \mathrm{mmol})$ of diethoxydimethylsilane and $11 \mathrm{mg}$ (2 mol\%) of $p$-TSA as a catalyst were added. The mixture was stirred using a magnetic stirrer for 24 hours at $100^{\circ} \mathrm{C}$ under a nitrogen atmosphere. At this point the mixture became homogeneous and light yellow in color. After this time had elapsed, dynamic vacuum was applied and the temperature was increased gradually from $130{ }^{\circ} \mathrm{C}$ to 180 ${ }^{\circ} \mathrm{C}$ for 8 hours and then the reaction was stopped after becoming very viscous as a light yellow sticky solid $(0.28 \mathrm{~g}, 42 \%$ yield $) .{ }^{1} \mathrm{H}$ NMR $\left(\mathrm{CDCl}_{3}\right): \delta \mathrm{ppm} 0.14-0.05$ $(\mathrm{m}, 6 \mathrm{H}), 0.80(\mathrm{~s}, 3 \mathrm{H}), 1.02(\mathrm{~s}, 3 \mathrm{H}), 1.03(\mathrm{~s}, 3 \mathrm{H}), 1.35-$ $1.43(\mathrm{~m}, 2 \mathrm{H}), 1.53-1.68(\mathrm{~m}, 1 \mathrm{H}), 1.85-2.02(\mathrm{~m}, 1 \mathrm{H})$, $2.03-2.17(\mathrm{~m}, 1 \mathrm{H}), 3.48(\mathrm{~d}, J=10.8 \mathrm{~Hz}, 1 \mathrm{H}), 3.52$ (dd, $J=10.3,8.3 \mathrm{~Hz}, 1 \mathrm{H}), 3.59$ (d, $J=10.8 \mathrm{~Hz}, 1 \mathrm{H}), 3.74$ (dd, $J=10.3,5.4 \mathrm{~Hz}, 1 \mathrm{H}$ ).

${ }^{13} \mathrm{C}$ NMR $\left(\mathrm{CDCl}_{3}\right): \delta \mathrm{ppm} 1.0,18.5,20.4,24.2,25.5$, 33.7, 44.0, 48.8, 50.2, 65.0, 69.2.

\section{Polyethylene hydroquinonoate silicon acetal (1 pot synthesis)}<smiles>C[Al]CCOc1ccc(OCCO[Si](C)(C)C)cc1</smiles>

Table S1, Entry 14. In a dry $50 \mathrm{~mL}$ round bottom flask, $1.75 \mathrm{~g}(8.83 \mathrm{mmol})$ of hydroquinone bis (2-hydroxyethyl) ether, $1.51 \mathrm{~mL}(8.83 \mathrm{mmol})$ of diethoxydimethylsilane and $34 \mathrm{mg}(2 \mathrm{~mol} \%)$ of $p$-TSA as the catalyst were combined. The mixture was then stirred under a nitrogen atmosphere for 24 hours at $110{ }^{\circ} \mathrm{C}$, at which it became clear and homogeneous. After this time, dynamic vacuum 
was applied for 8 hours by gradually changing the temperature from $110^{\circ} \mathrm{C}$ to $180^{\circ} \mathrm{C}$. After 8 hours under vacuum, the mixture became viscous and stirring was very slow, and the reaction was stopped. A white solid was isolated (1.88 g, 85\% yield). ${ }^{1} \mathrm{H}$ NMR (benzene- $d_{6}$ ): $\delta$ ppm $0.23(\mathrm{~s}, 6 \mathrm{H}), 3.81(\mathrm{t}, J=5.0 \mathrm{~Hz}, 4 \mathrm{H}), 3.93(\mathrm{t}, J=$ $5.0 \mathrm{~Hz}, 4 \mathrm{H}), 6.81(\mathrm{~s}, 4 \mathrm{H}) .{ }^{13} \mathrm{C}$ NMR (benzene- $\left.d_{6}\right): \delta \mathrm{ppm}$ $-2.4,62.0,70.2,116.1,154.1$.

\section{Polyethylene terephthalate silicon acetal}<smiles>C[Al](C)OCCOC(=O)c1ccc(C(=O)OCCO[Si](C)(C)C)cc1</smiles>

Table S1, Entry 15. In a dry $50 \mathrm{~mL}$ round bottom flask equipped with a magnetic stir bar, $2.00 \mathrm{~g}(7.87 \mathrm{mmol})$ of bis(2-hydroxyethyl ether) terephthalate, $1.62 \mathrm{~mL}(9.44$ $\mathrm{mmol})$ of diethoxydimethylsilane and $30 \mathrm{mg}(2 \mathrm{~mol} \%)$ of $p$-TSA were combined. The mixture was stirred in the melt at $100{ }^{\circ} \mathrm{C}$ under a nitrogen atmosphere for 24 hours. After that, dynamic vacuum was applied to remove ethanol for 8 hours. The temperature was then increased to $130{ }^{\circ} \mathrm{C}$ and vacuum applied for 24 hours. A white solid was isolated $(1.82 \mathrm{~g}, 75 \%$ yield $) .{ }^{1} \mathrm{H} \mathrm{NMR}\left(\mathrm{CDCl}_{3}\right): \delta$ ppm $-0.09-0.32$ (m, $6 \mathrm{H}), 4.12-3.90$ (m, $4 \mathrm{H}), 4.63-$ $4.40(\mathrm{~m}, 4 \mathrm{H}), 8.11(\mathrm{~s}, 4 \mathrm{H}) .{ }^{13} \mathrm{C} \mathrm{NMR}\left(\mathrm{CDCl}_{3}\right): \delta \mathrm{ppm}$ $1.0,61.2,67.0,129.7,133.8,166.0$.

\section{Polyethylene hydroquinonoate silicon acetal (2 pot synthesis)}

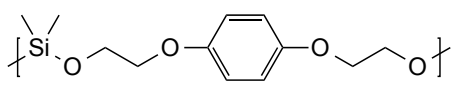

\section{Monomer synthesis}

\section{1,4-bis(2-((methoxydimethylsilyl)oxy)ethoxy)benzene}<smiles>CO[Si](C)(C)OCCOc1ccc(OCCO[Si](C)(C)OC)cc1</smiles>

In a dry two-neck $100 \mathrm{~mL}$ flask, $2.00 \mathrm{~g}(10.09 \mathrm{mmol})$ of hydroquinone bis(2-hydroxyethyl) ether was combined with dimethylmethoxychlorosilane $(3.75 \mathrm{~mL}, 24.22$ $\mathrm{mmol})$ in dry tetrahydrofuran $(30 \mathrm{~mL}) .3 .66 \mathrm{~mL}(26.23$ $\mathrm{mmol}$ ) of dry triethylamine were added drop-wise over a 5 minute period using an addition funnel. The resulting mixture was allowed to stir overnight at room temperature. The white suspension was quenched with saturated sodium bicarbonate $(15 \mathrm{~mL})$ twice and the organics were washed with brine $(15 \mathrm{~mL})$ twice, followed by drying over magnesium sulfate and the removal of tetrahydrofuran in vacuo. A light yellowish oil was obtained $\left(2.25 \mathrm{~g}, 60 \%\right.$ yield). ${ }^{1} \mathrm{H}$ NMR $\left(\mathrm{CDCl}_{3}\right): \delta \mathrm{ppm}$ $-0.45-0.35(\mathrm{~m}, 12 \mathrm{H}), 3.51(\mathrm{~s}, 6 \mathrm{H}), 3.96-4.08(\mathrm{~m}, 8$ $\mathrm{H}), 6.78-6.87(\mathrm{~m}, 4 \mathrm{H}) .{ }^{13} \mathrm{C} \mathrm{NMR}\left(\mathrm{CDCl}_{3}\right): \delta \mathrm{ppm}-3.6$, $50.1,61.3,69.6,115.4,153.1$.

\section{Polymer Synthesis}

Table S1, Entry 16. In a dry $50 \mathrm{~mL}$ round bottom flask, $1.60 \mathrm{~g} \quad(4.27 \mathrm{mmol})$ of 1,4-bis(2((methoxydimethylsilyl)oxy)ethoxy)-benzene and $16 \mathrm{mg}$ (2 mol\%) of methanesulfonic acid as a catalyst were added. The mixture was stirred at $110{ }^{\circ} \mathrm{C}$ for 4 hours under nitrogen atmosphere. After that time had elapsed, dynamic vacuum was applied for 1 hour at $110{ }^{\circ} \mathrm{C}, 4$ hours at $130{ }^{\circ} \mathrm{C}$, then 5 hours at $170{ }^{\circ} \mathrm{C}$. A white solid was isolated $(1.01 \mathrm{~g}, 93 \%$ yield $) .{ }^{1} \mathrm{H} \mathrm{NMR}\left(\mathrm{CDCl}_{3}\right): \delta$ ppm $0.08(\mathrm{~s}, 6 \mathrm{H}), 3.95$ (t, $J=4.5 \mathrm{~Hz}, 4 \mathrm{H}), 4.05$ (t, $J=$ $4.5 \mathrm{~Hz}, 4 \mathrm{H}), 6.87(\mathrm{~s}, 4 \mathrm{H}) .{ }^{13} \mathrm{C} \mathrm{NMR}\left(\mathrm{CDCl}_{3}\right): \delta \mathrm{ppm} \mathrm{1.0,}$ $61.6,69.8,115.6,153.1$. 


\section{Summary of Polymerization Data}

Table S1. Polysilicon acetal synthesis via $\mathrm{HCl}$ elimination (entries 1-4) versus silicon acetal metathesis polymerization (entries 5-16)

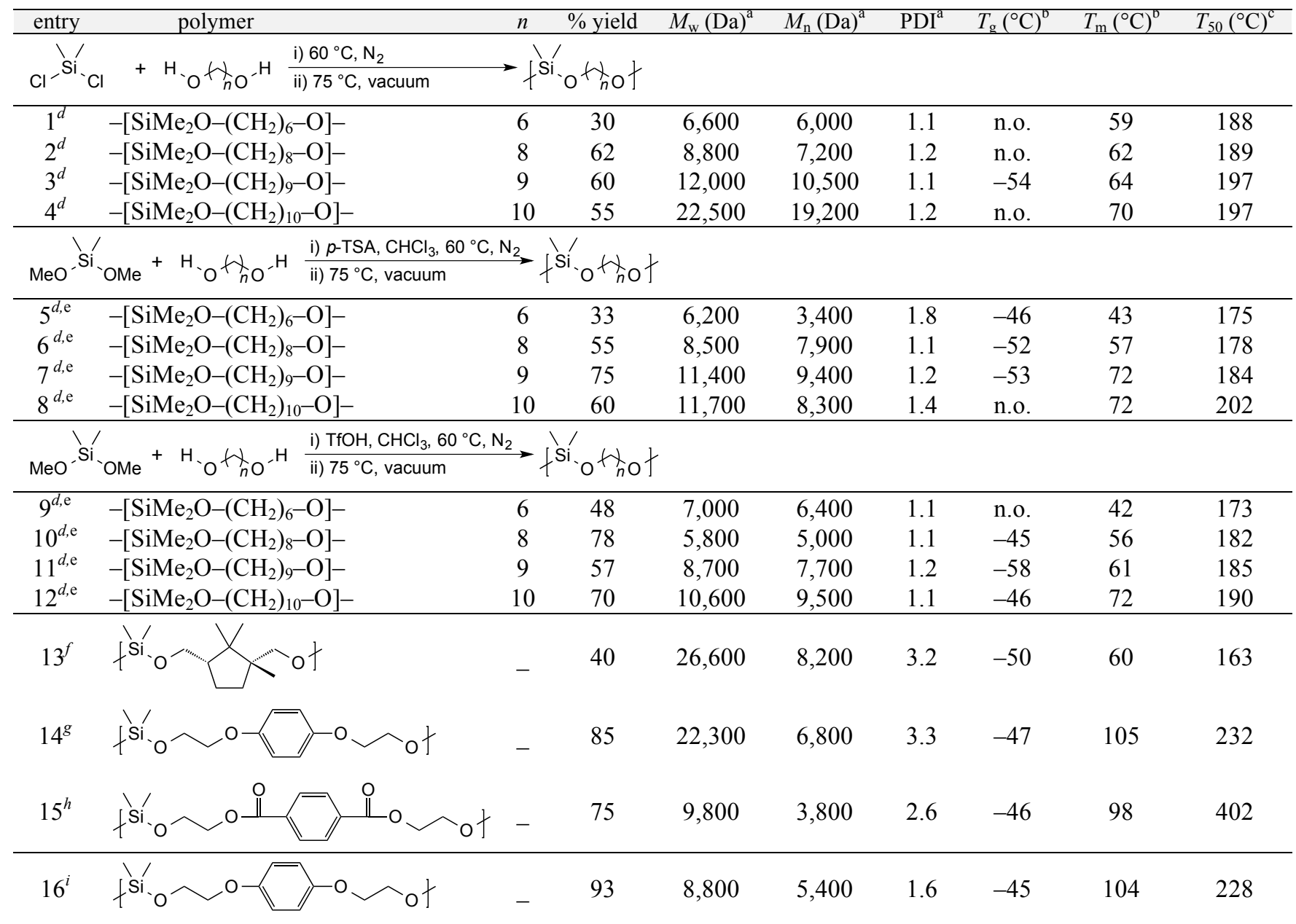

${ }^{a}$ Obtained by GPC in dimethylformamide (entries 1-12) or tetrahydrofuran (entries 13-16) versus polystyrene (PS) standards. ${ }^{b}$ Determined by DSC; n.o. = not observed. ${ }^{c}$ Temperature at which $50 \%$ mass loss is observed under nitrogen by thermogravimetric analysis (TGA). ${ }^{d}$ Polymerization at $60{ }^{\circ} \mathrm{C}$ under nitrogen for 24 hours, followed by vacuum for 2.5 hours and then vacuum at $75{ }^{\circ} \mathrm{C}$ for 3.5 hours. ${ }^{e}$ Chloroform solvent and $1 \mathrm{~mol} \%$ acid catalyst added. ${ }^{f}$ Diol, diethoxydimethylsilane, and 2 mol $\% p$-TSA at $100{ }^{\circ} \mathrm{C}$ for 24 hours, followed by 8 hours of vacuum at $130-180{ }^{\circ} \mathrm{C}$. ${ }^{g}$ Diol, diethoxydimethylsilane, and 2 mol\% $p$-TSA at $110{ }^{\circ} \mathrm{C}$ for 24 hours, followed by 8 hours of vacuum at $110-200{ }^{\circ} \mathrm{C}$. ${ }^{h}$ Diol, diethoxydimethylsilane, and $2 \mathrm{~mol} \% \mathrm{MeSO}_{3} \mathrm{H}$ at $100{ }^{\circ} \mathrm{C}$ for 24 hours, followed by 8 hours of vacuum at $130{ }^{\circ} \mathrm{C}$. ${ }^{i} 1,4$-bis(2-((methoxydimethylsilyl)oxy)ethoxy)benzene and 2 mol\% $\mathrm{MeSO}_{3} \mathrm{H}$ at $110{ }^{\circ} \mathrm{C}$ for 4 hours under nitrogen, followed by vacuum for 1 hour at $110{ }^{\circ} \mathrm{C}, 4$ hours at $130{ }^{\circ} \mathrm{C}$, then 5 hours at $170{ }^{\circ} \mathrm{C}$. 


\section{Gel Permeation Chromatography (GPC) Data}

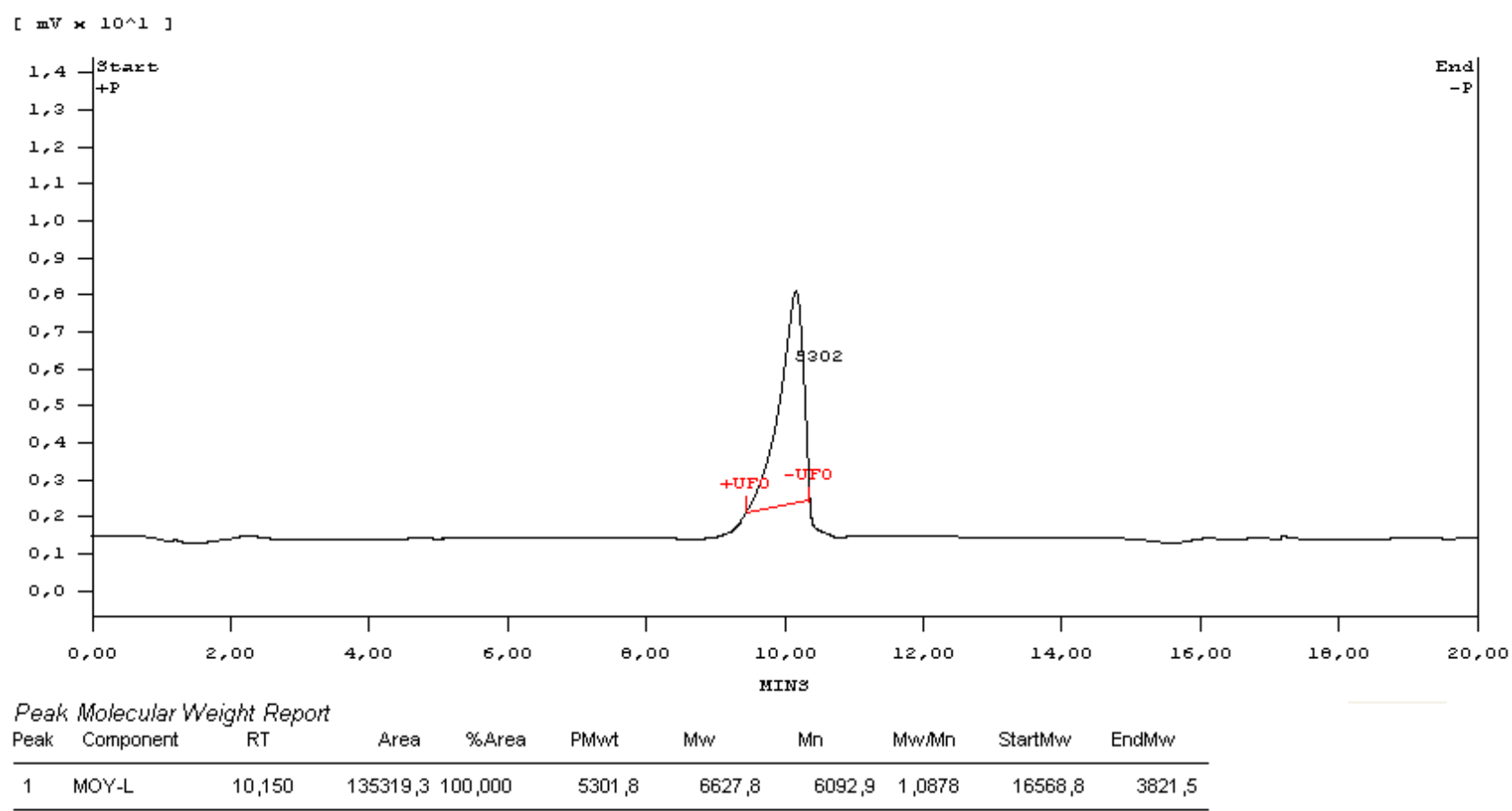

Figure S1. GPC Chromatogram of polyhexylene silicon acetal (Table S1, entry 1).

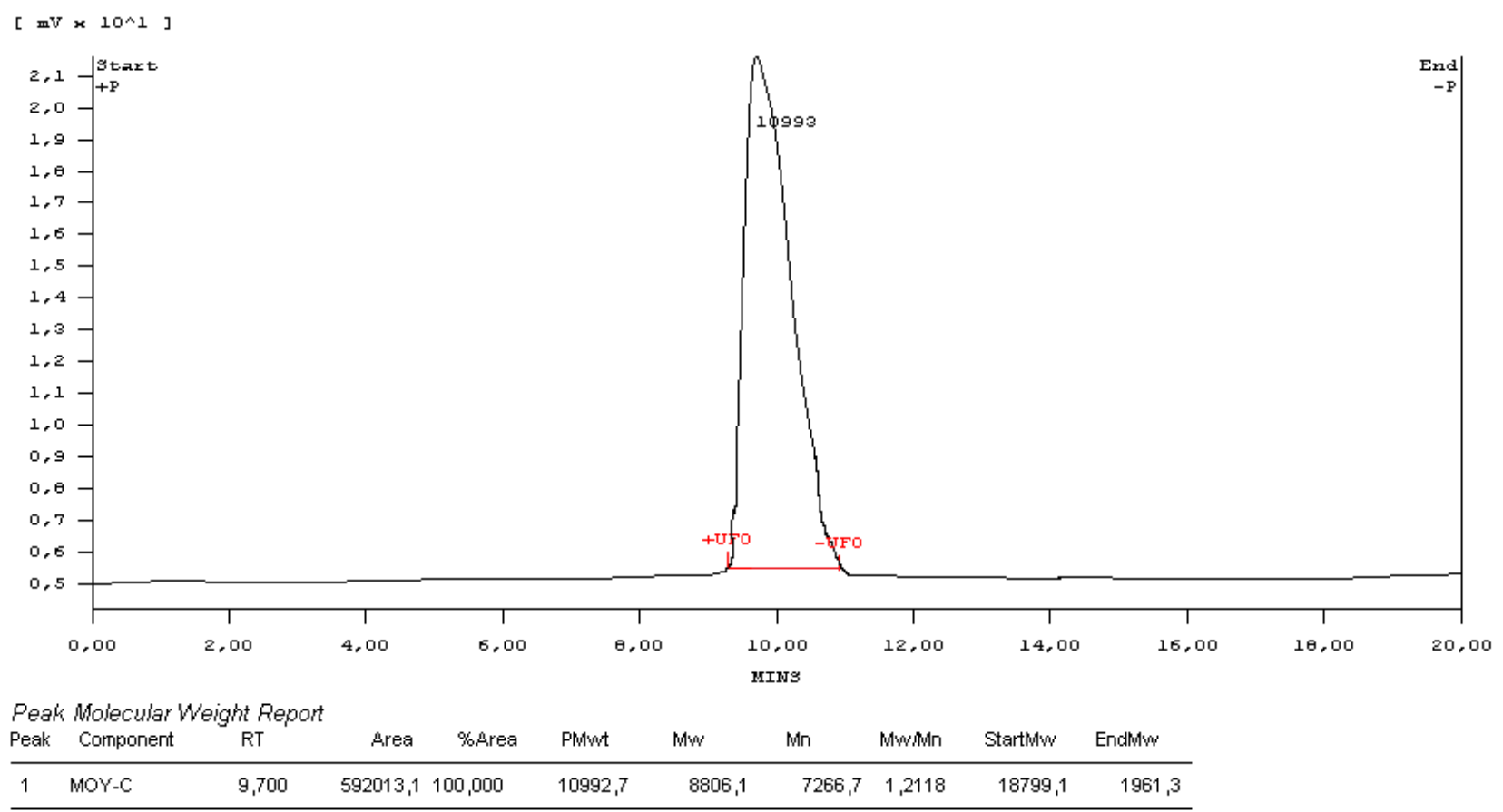

Figure S2. GPC Chromatogram of polyoctylene silicon acetal (Table S1, entry 2). 


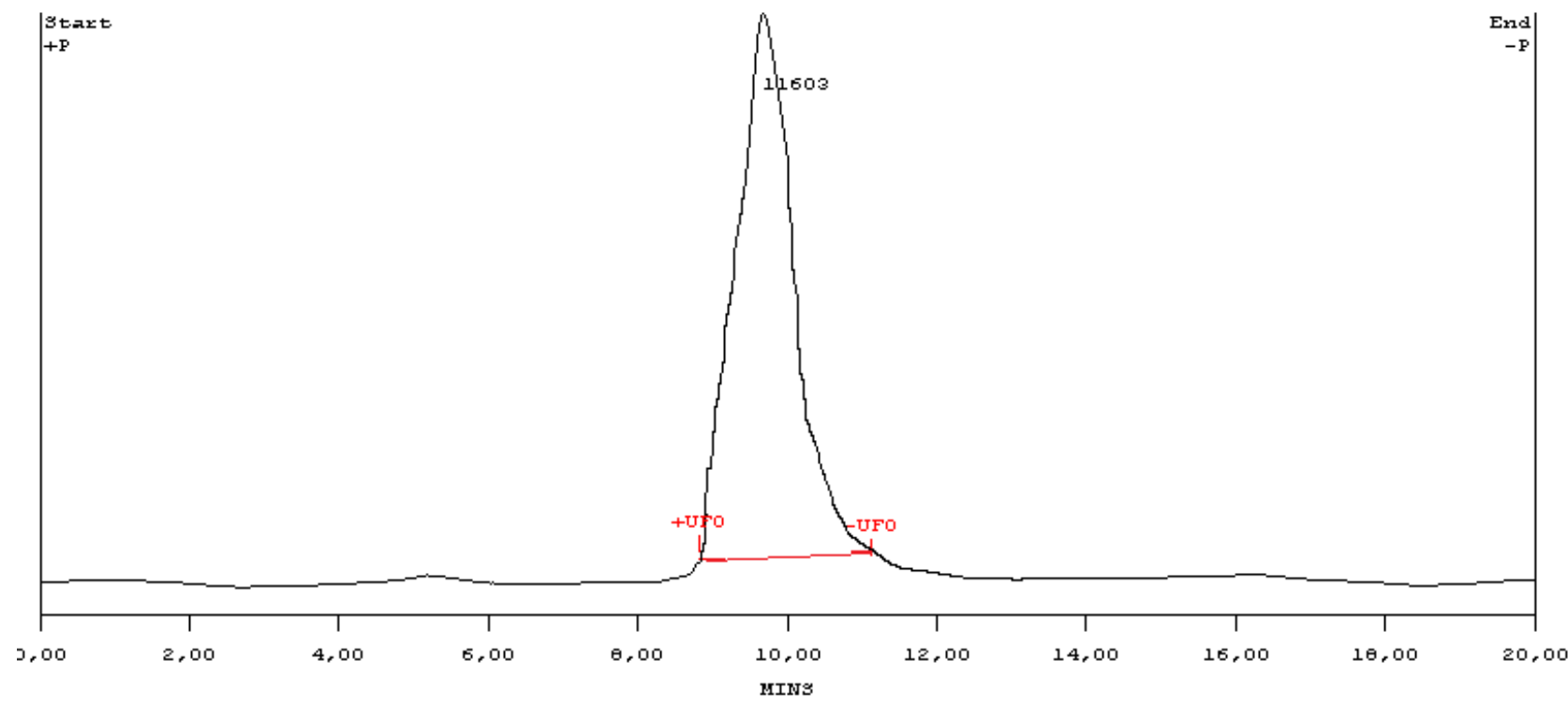

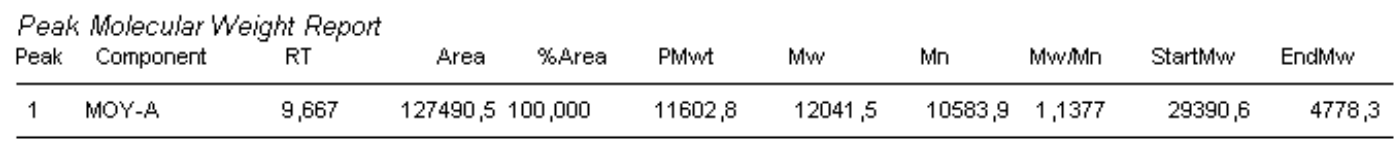

Figure S3. GPC Chromatogram of polynonylene silicon acetal (Table S1, entry 3).

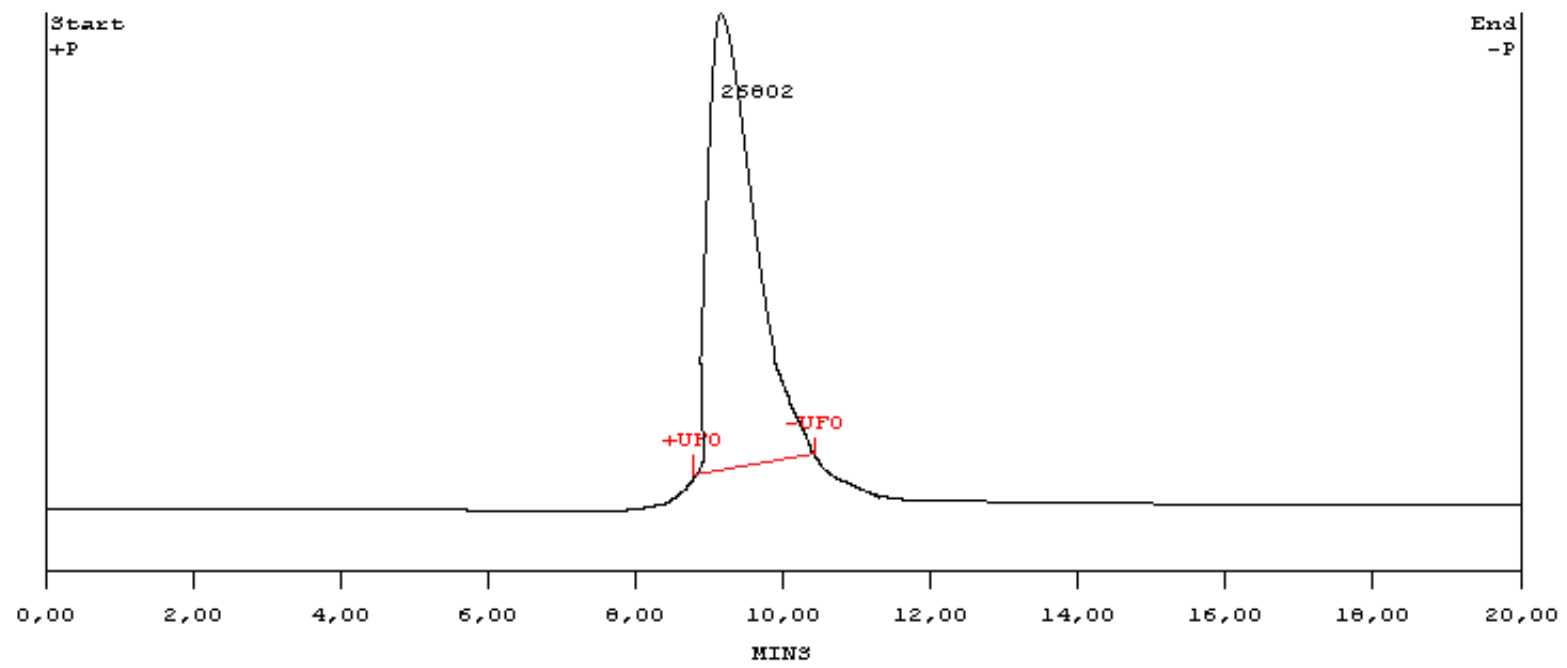

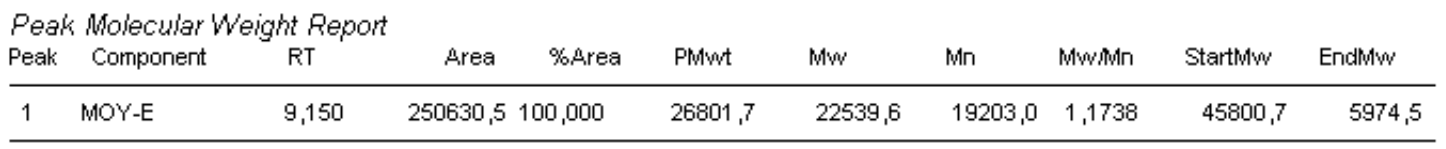

Figure S4. GPC Chromatogram of polydecylene silicon acetal (Table S1, entry 4).

S7 


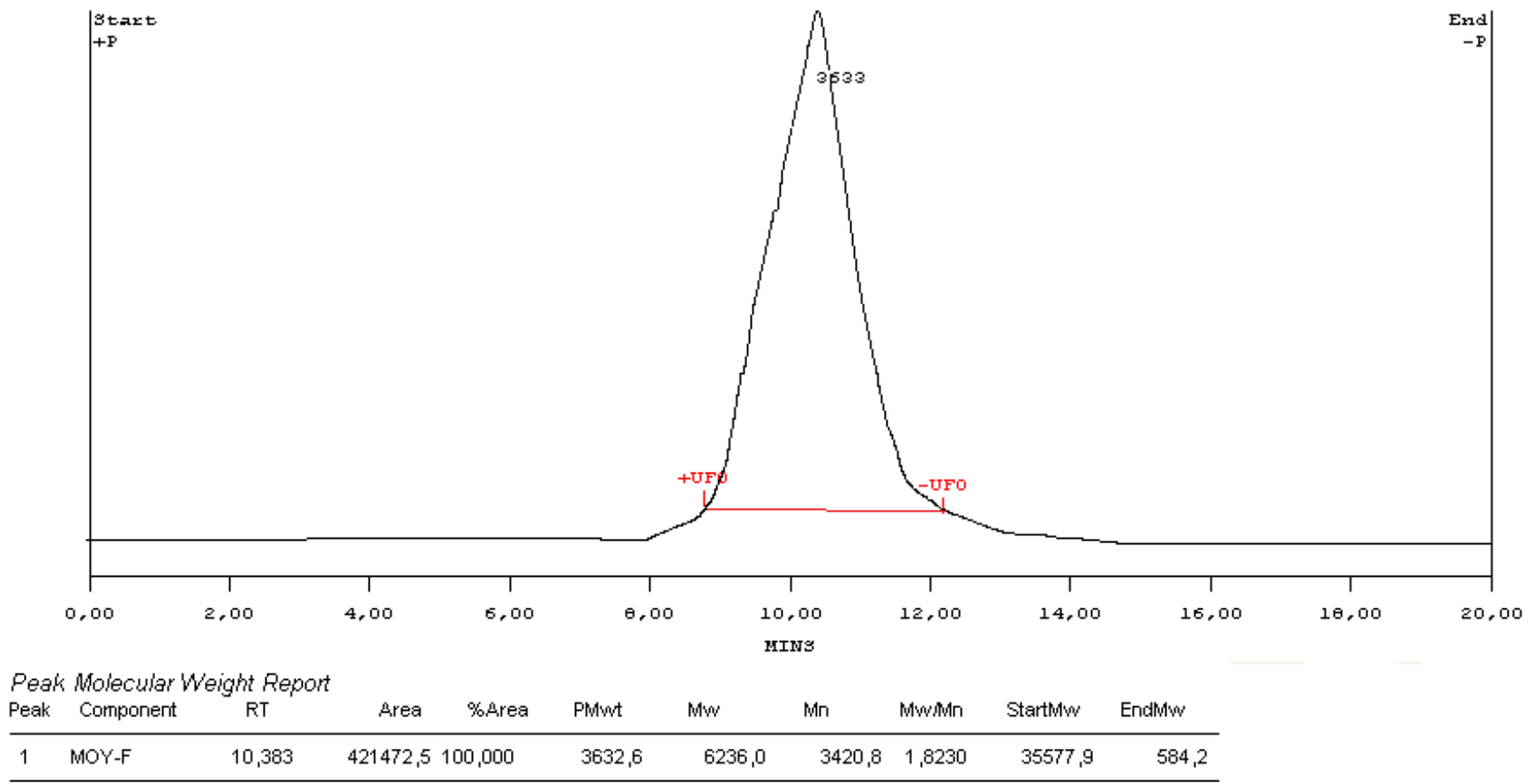

Figure S5. GPC Chromatogram of polyhexylene silicon acetal (Table S1, entry 5).

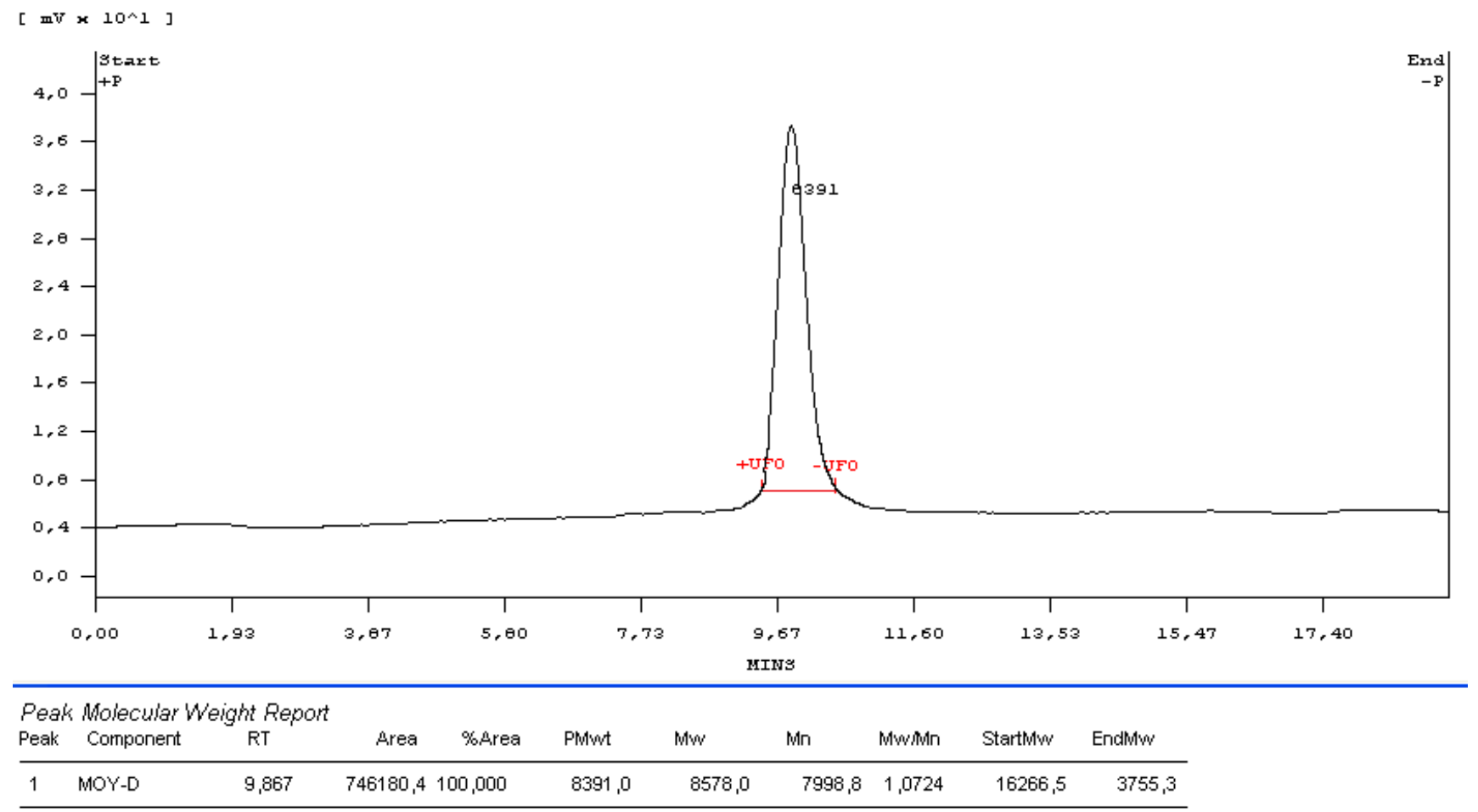

Figure S6. GPC Chromatogram of polyoctylene silicon acetal (Table S1, entry 6). 


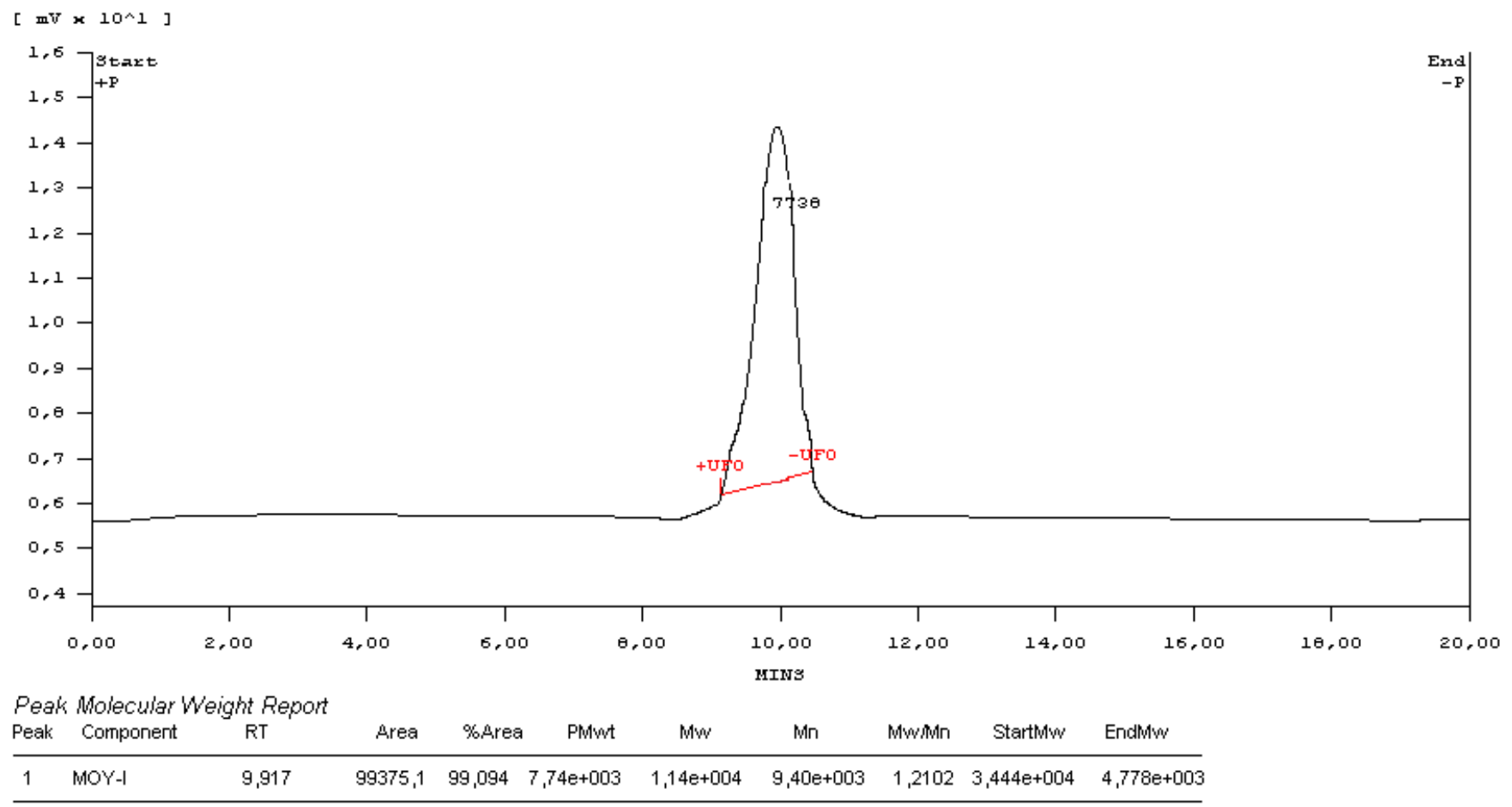

Figure S7. GPC Chromatogram polynonylene silicon acetal (Table S1, entry 7).

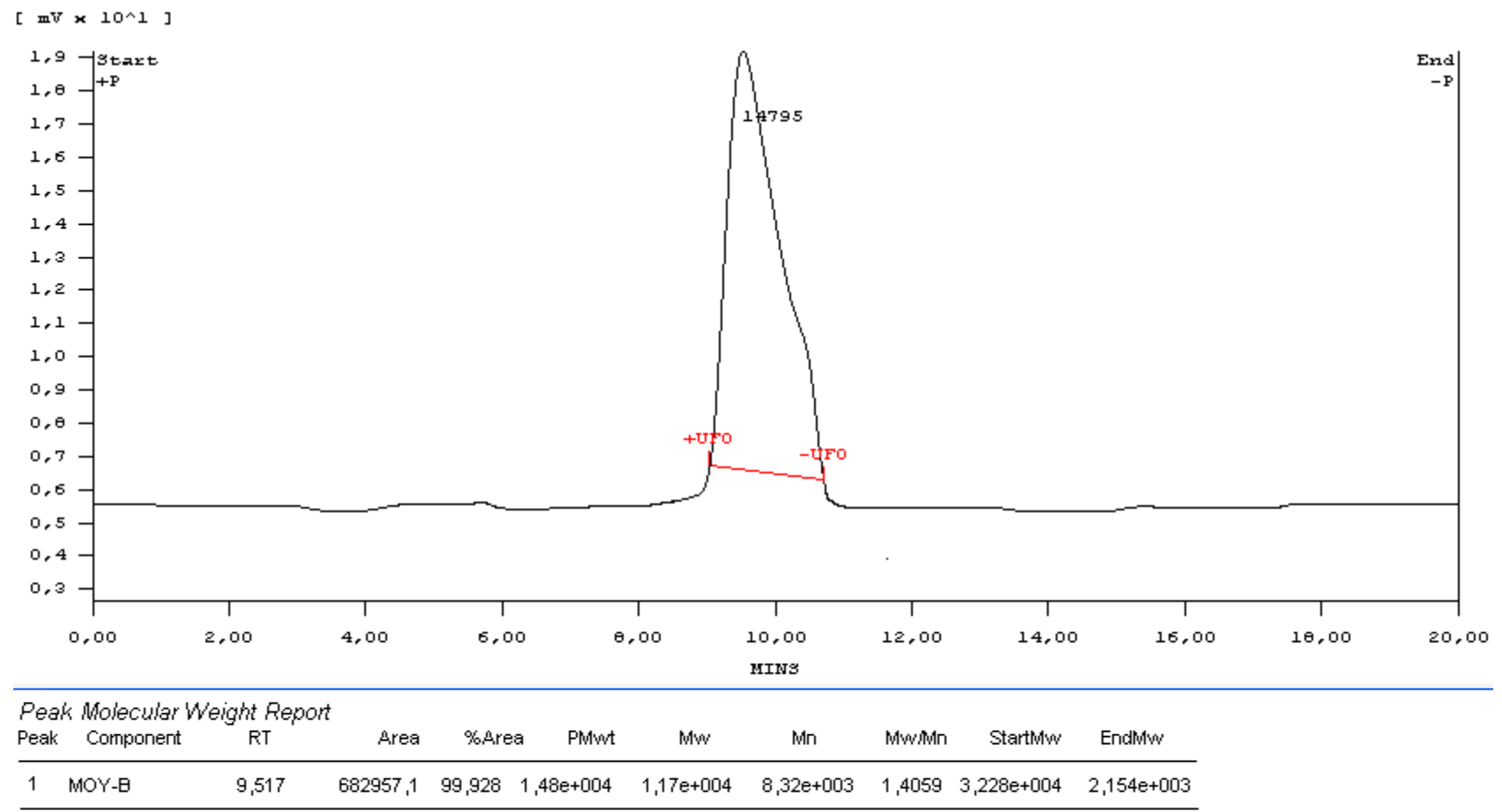

Figure S8. GPC Chromatogram of polydecylene silicon acetal (Table S1, entry 8). 


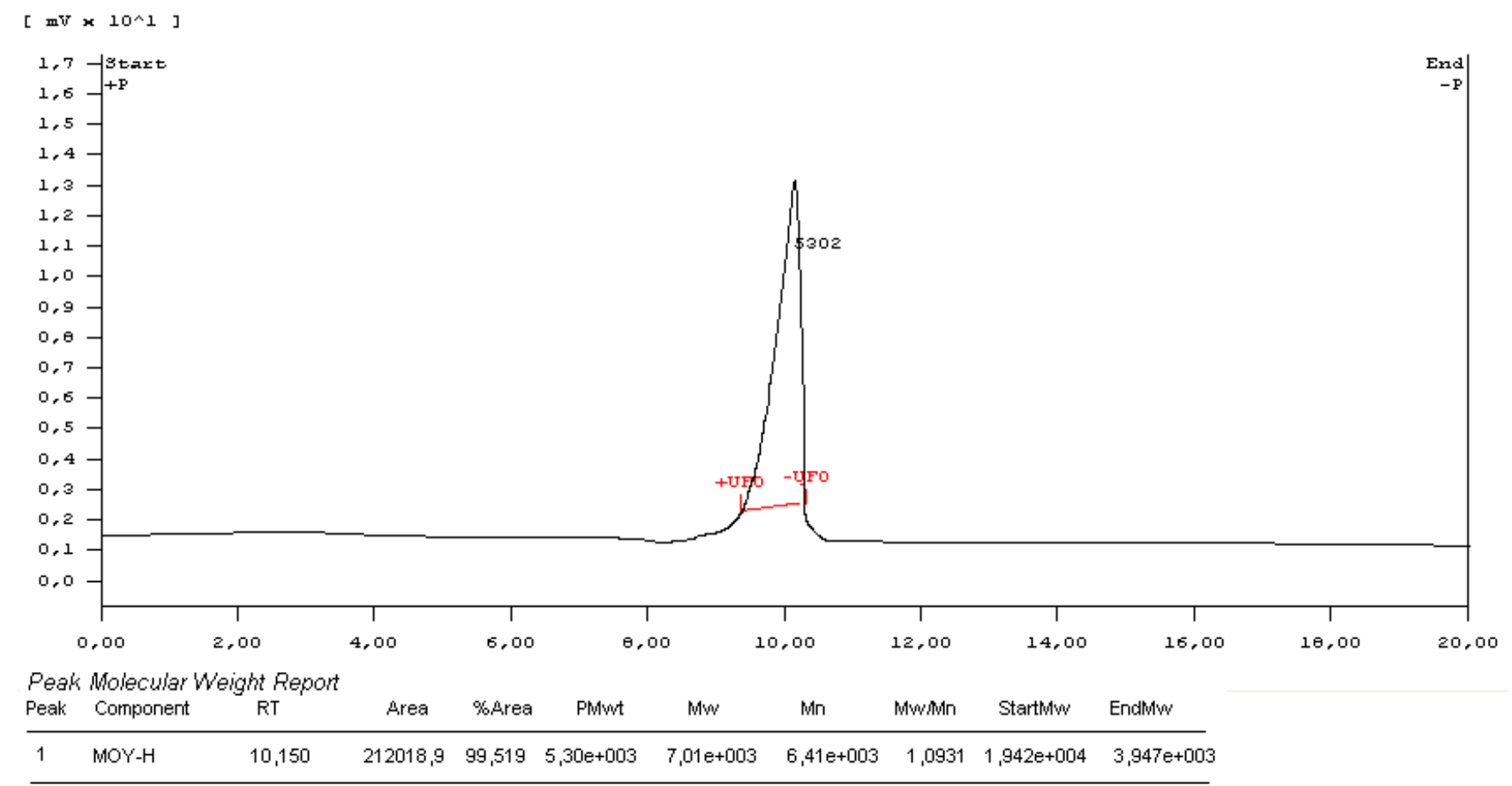

Figure S9. GPC Chromatogram of polyhexylene silicon acetal (Table S1, entry 9).

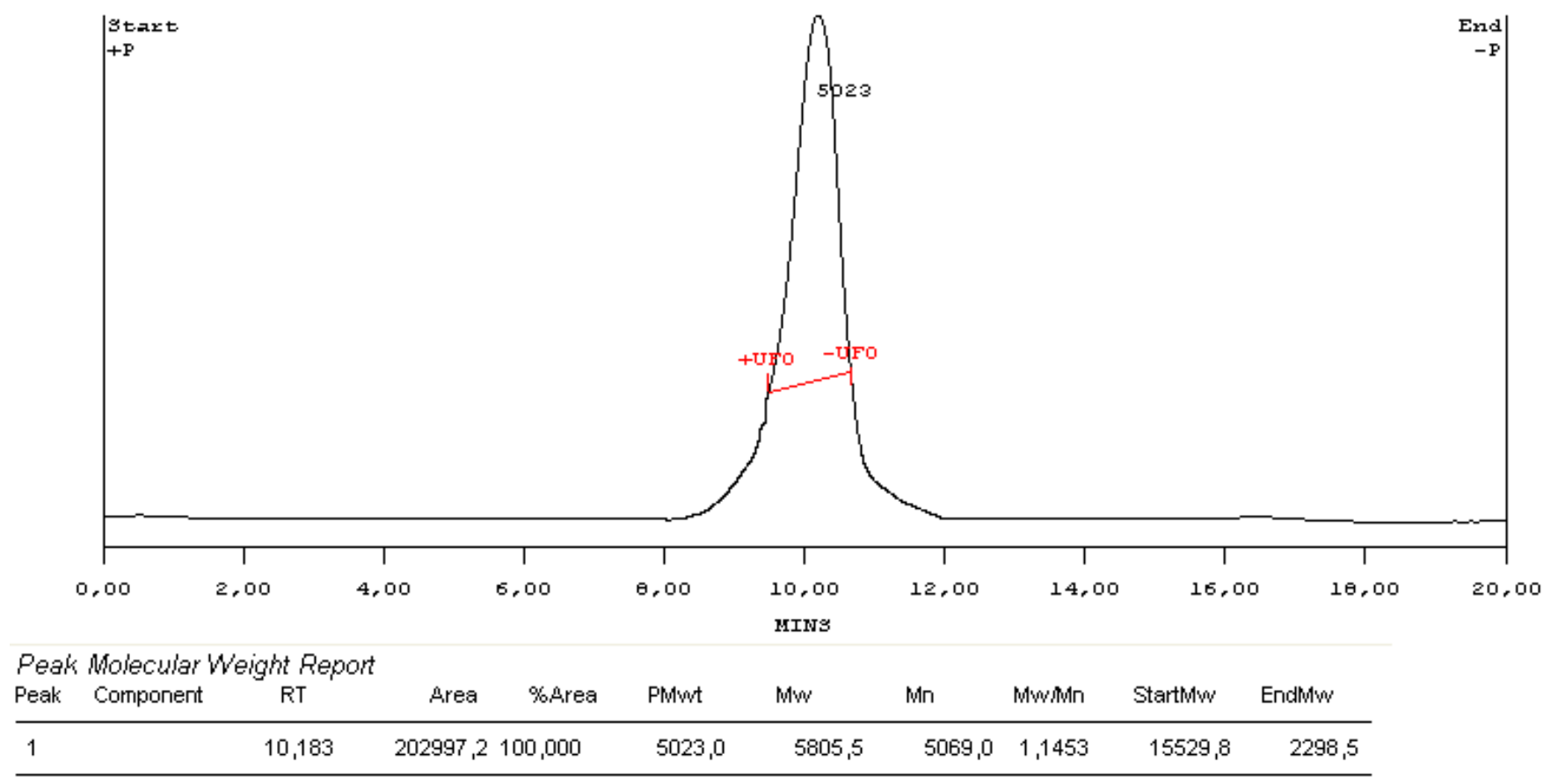

Figure S10. GPC Chromatogram of polyoctlyne silicon acetal (Table S1, entry 10). 


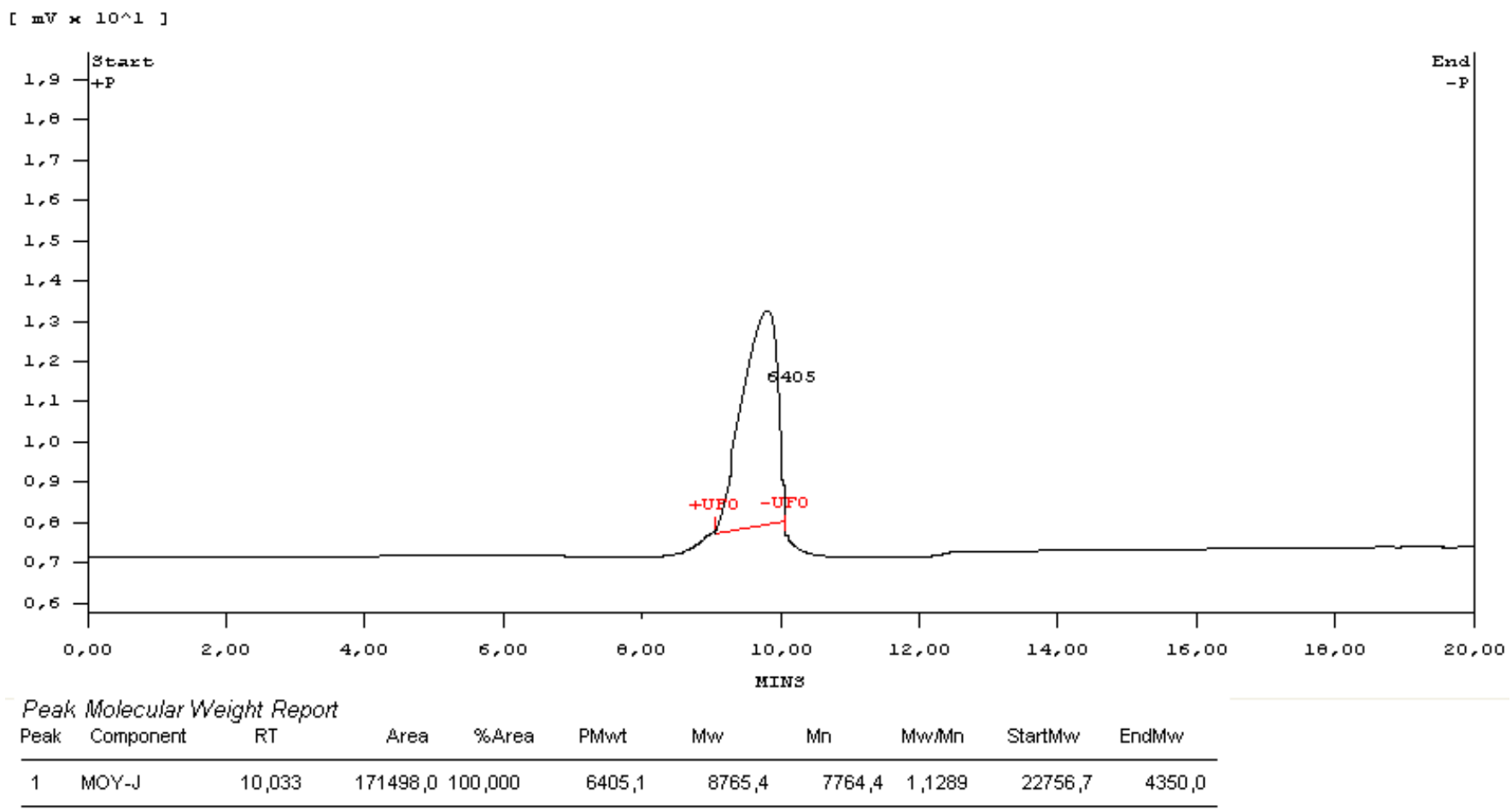

Figure S11. GPC Chromatogram of polynonylene silicon acetal (Table S1, entry 11).

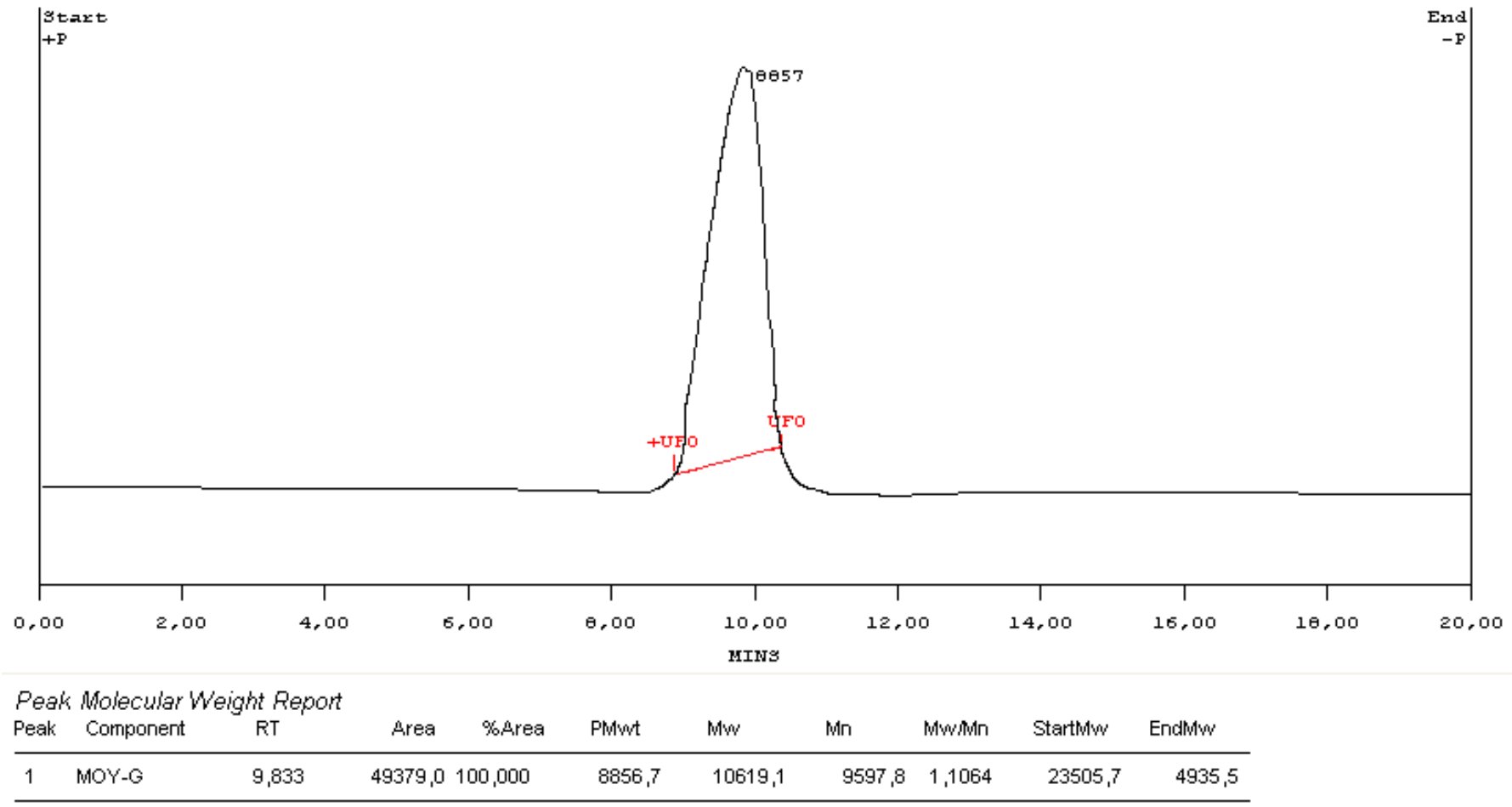

Figure S12. GPC Chromatogram of polydecylene silicon acetal (Table S1, entry 12). 


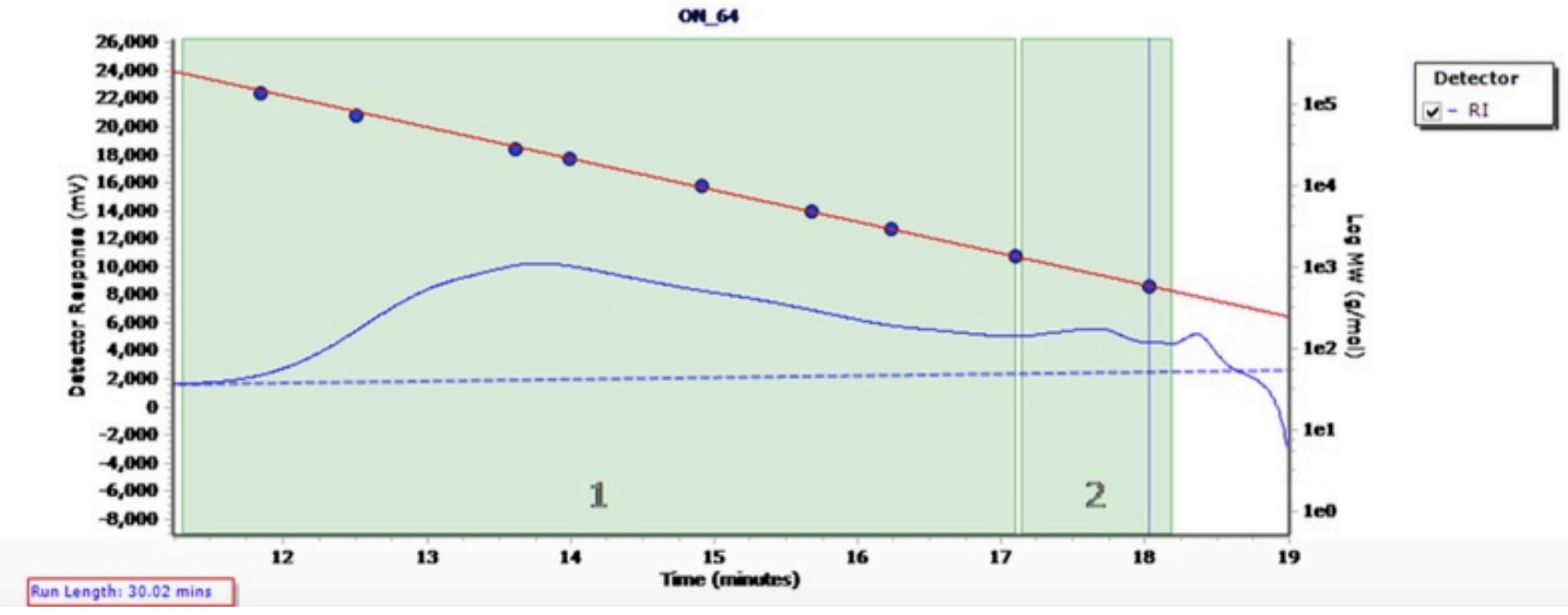

Molecular Weight Averages

\begin{tabular}{|l|r|r|r|r|r|r|r|}
\hline Peak & \multicolumn{1}{|c|}{ Mp } & \multicolumn{1}{c|}{ Mn } & \multicolumn{1}{c|}{ Mw } & \multicolumn{1}{c|}{ Mz } & \multicolumn{1}{c|}{ Mz+1 } & \multicolumn{1}{c|}{ Mv } & PD \\
\hline Peak 1 & 26656 & 8253 & 26644 & 55396 & 82892 & 51431 & 3.228 \\
\hline Peak 2 & 833 & 812 & 868 & 923 & 975 & 915 & 1.069 \\
\hline
\end{tabular}

Figure S13. GPC Chromatogram of polycamphoric silicon acetal (Table S1, entry 13).

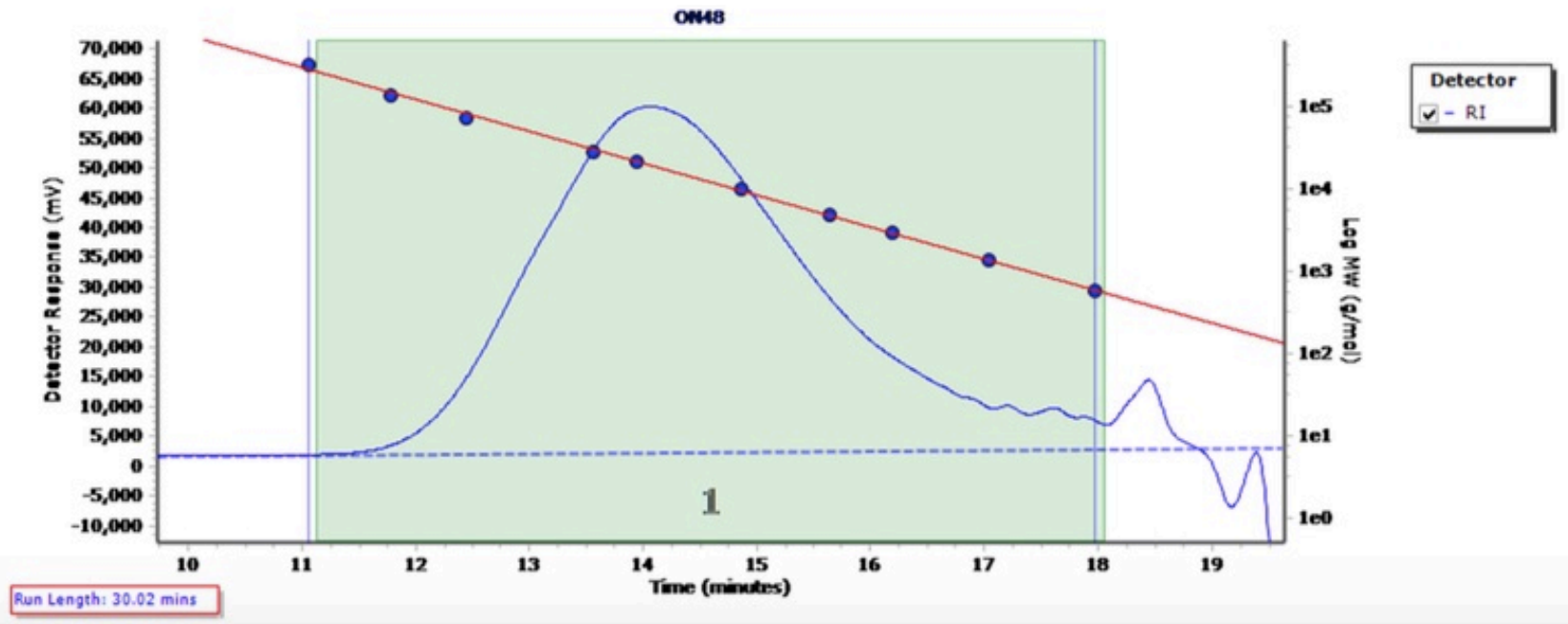

\section{Molecular Weight Averages}

\begin{tabular}{|c|c|c|c|c|c|c|r|}
\hline Peak & Mp & Mn & Mw & Mz & Mz+1 & \multicolumn{1}{|c|}{ Mv } & PD \\
\hline Peak 1 & 19561 & 6851 & 22379 & 44789 & 70532 & 41384 & 3.267 \\
\hline
\end{tabular}

Figure S14. GPC Chromatogram of polyethylene hydroquinonoate silicon acetal (Table S1, entry 14). 


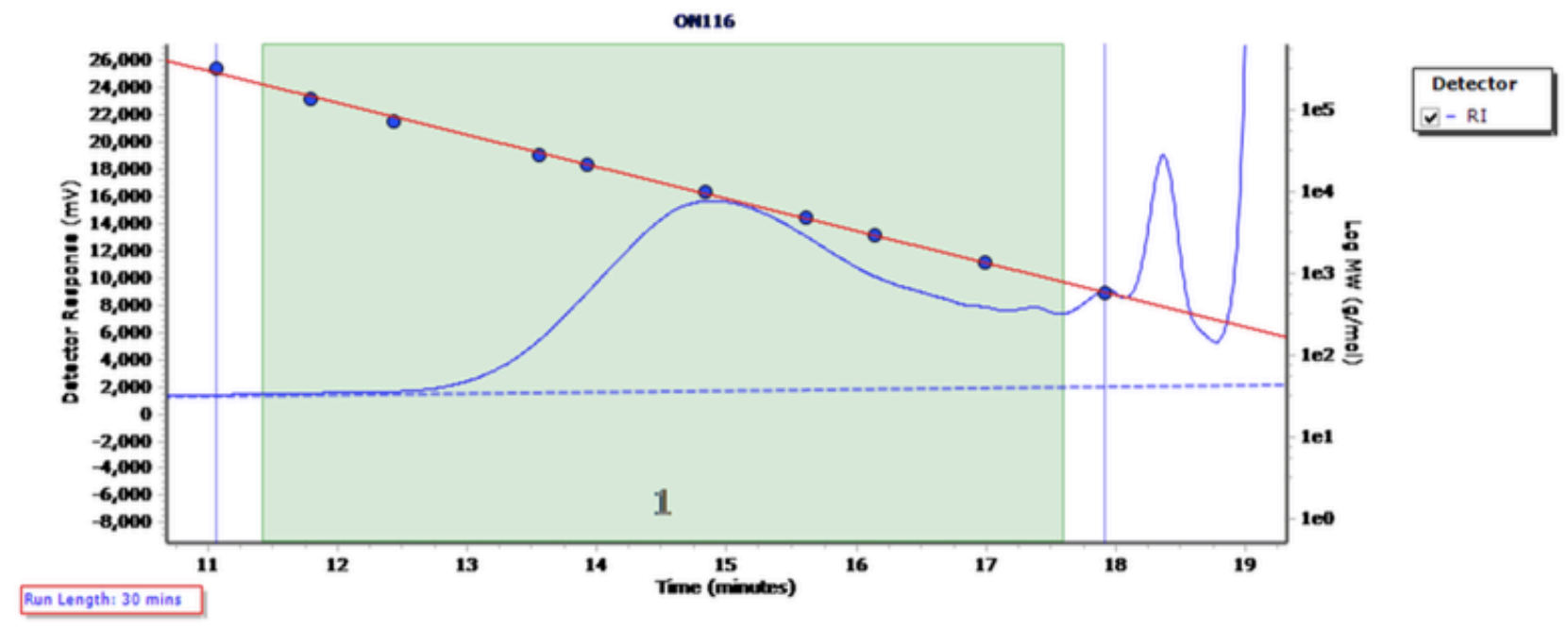

\begin{tabular}{|c|c|c|c|c|c|c|c|}
\hline Peak & $\mathrm{Mp}$ & $\mathrm{Mn}$ & Mw & \begin{tabular}{|l|}
$\mathrm{Mz}$ \\
\end{tabular} & $\mathrm{Mz}+1$ & Mv & PD \\
\hline Peak 1 & 9181 & 3816 & 9844 & 24028 & 62137 & 20869 & 2.58 \\
\hline
\end{tabular}

Figure S15. GPC Chromatogram of polyethylene terephthalate silicon acetal (Table S1, entry 15).

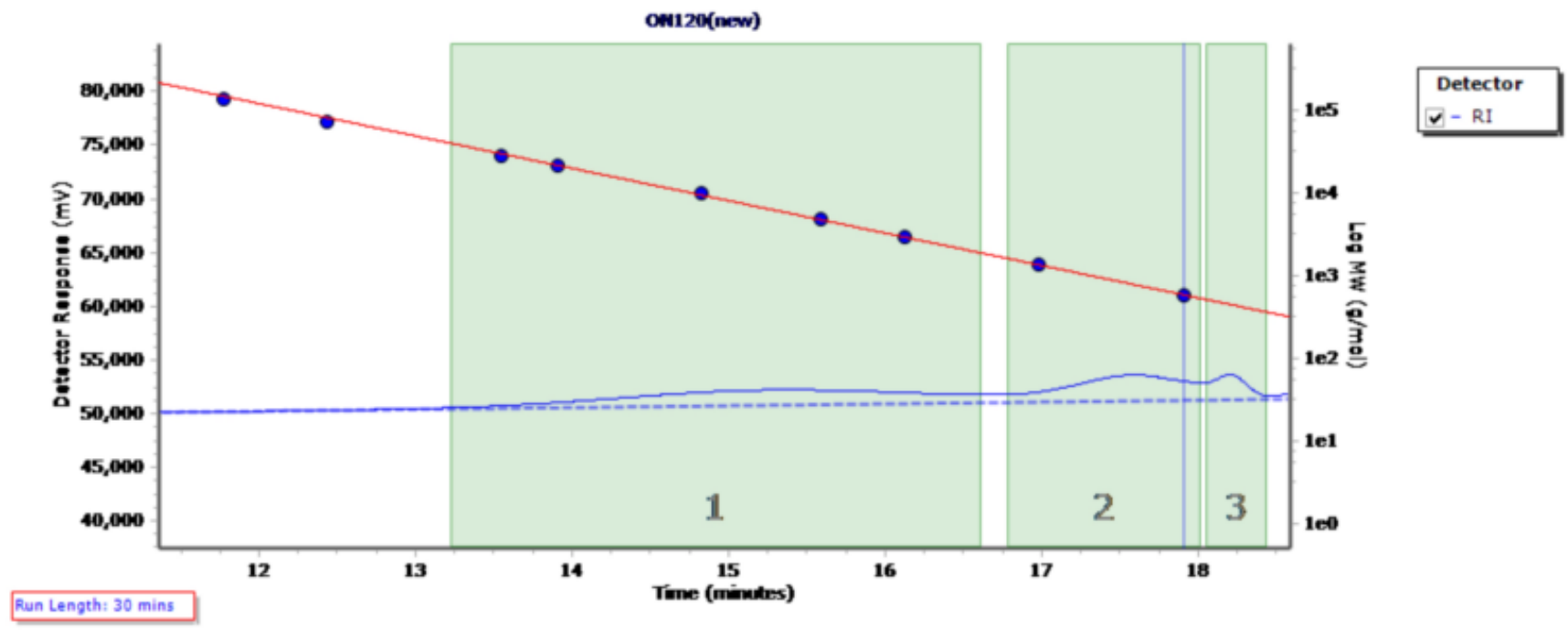

Molecular Weight Averages
\begin{tabular}{|l|r|r|r|r|r|r|r|}
\hline \multicolumn{1}{|c|}{ Peak } & \multicolumn{1}{|c|}{ Mp } & \multicolumn{1}{|c|}{ Mn } & \multicolumn{1}{c|}{ Mw } & \multicolumn{1}{c|}{ Mz } & Mz+1 & \multicolumn{1}{c|}{ Mv } & \multicolumn{1}{c|}{ P } \\
\hline Peak 1 & 6248 & 5387 & 8764 & 14099 & 19931 & 13256 & 1.627 \\
\hline Peak 2 & 790 & 828 & 896 & 974 & 1055 & 962 & 1.082 \\
\hline Peak 3 & 453 & 448 & 451 & 454 & 457 & 454 & 1.007 \\
\hline
\end{tabular}

Figure S16. GPC Chromatogram of polyethylene hydroquinonoate silicon acetal (Table S1, entry 16). 


\section{Differential Scanning Calorimetry (DSC) Thermograms}

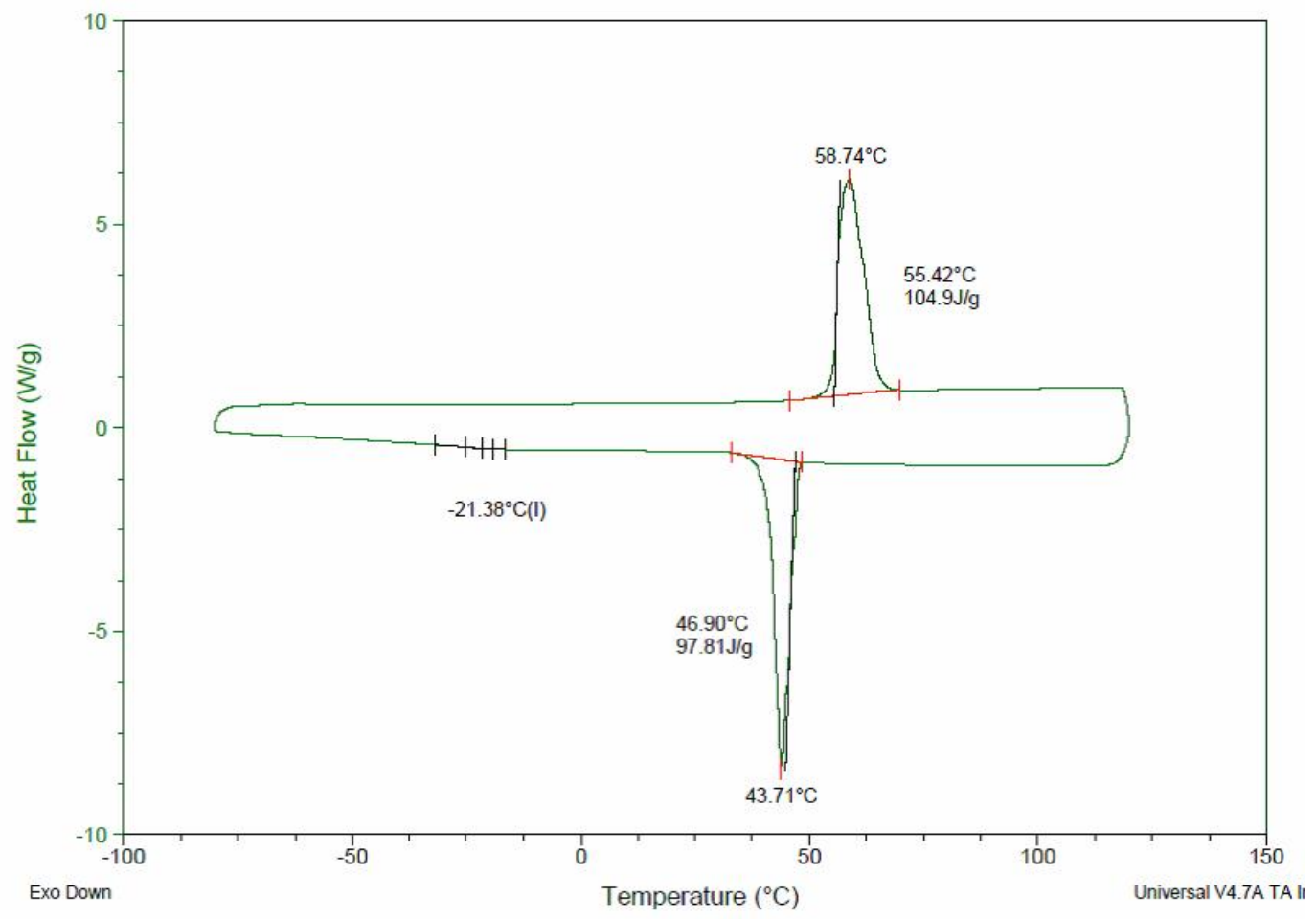

Figure S17. DSC Thermogram of polyhexylene silicon acetal (Table S1, entry 1).

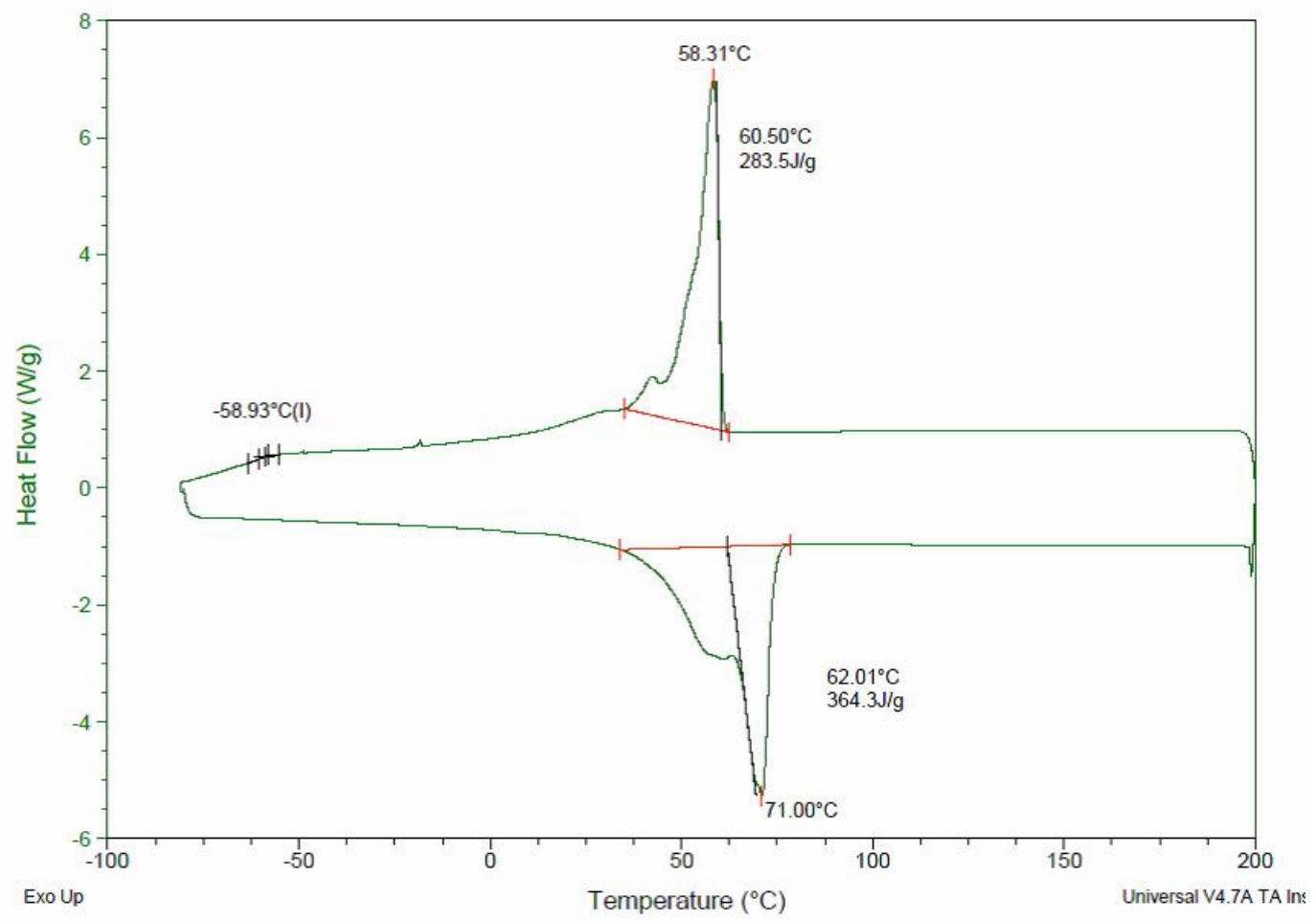

Figure S18. DSC Thermogram of polyoctylene silicon acetal (Table S1, entry 2). 


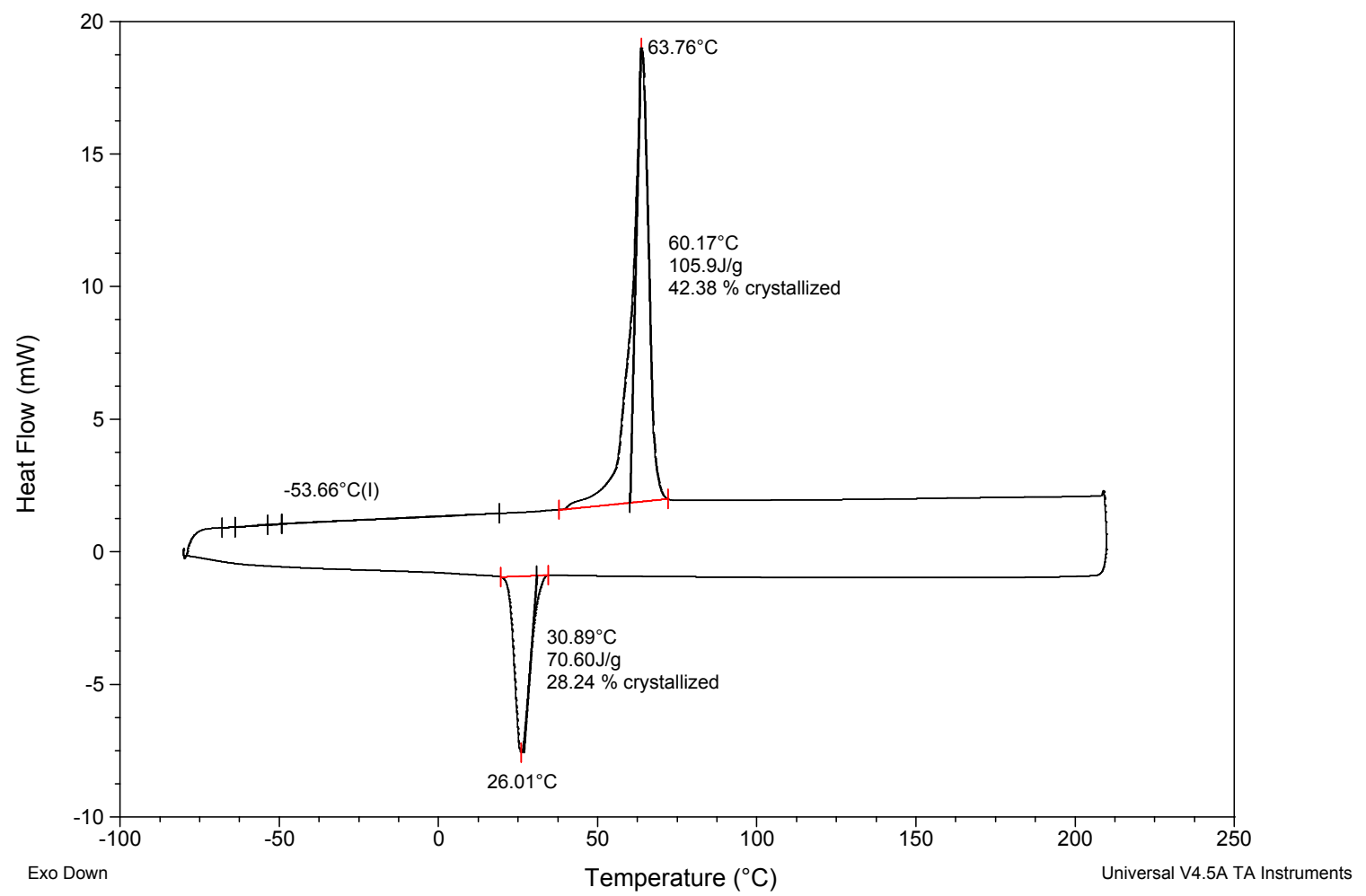

Figure S19. DSC Thermogram of polyononylene silicon acetal (Table S1, entry 3).

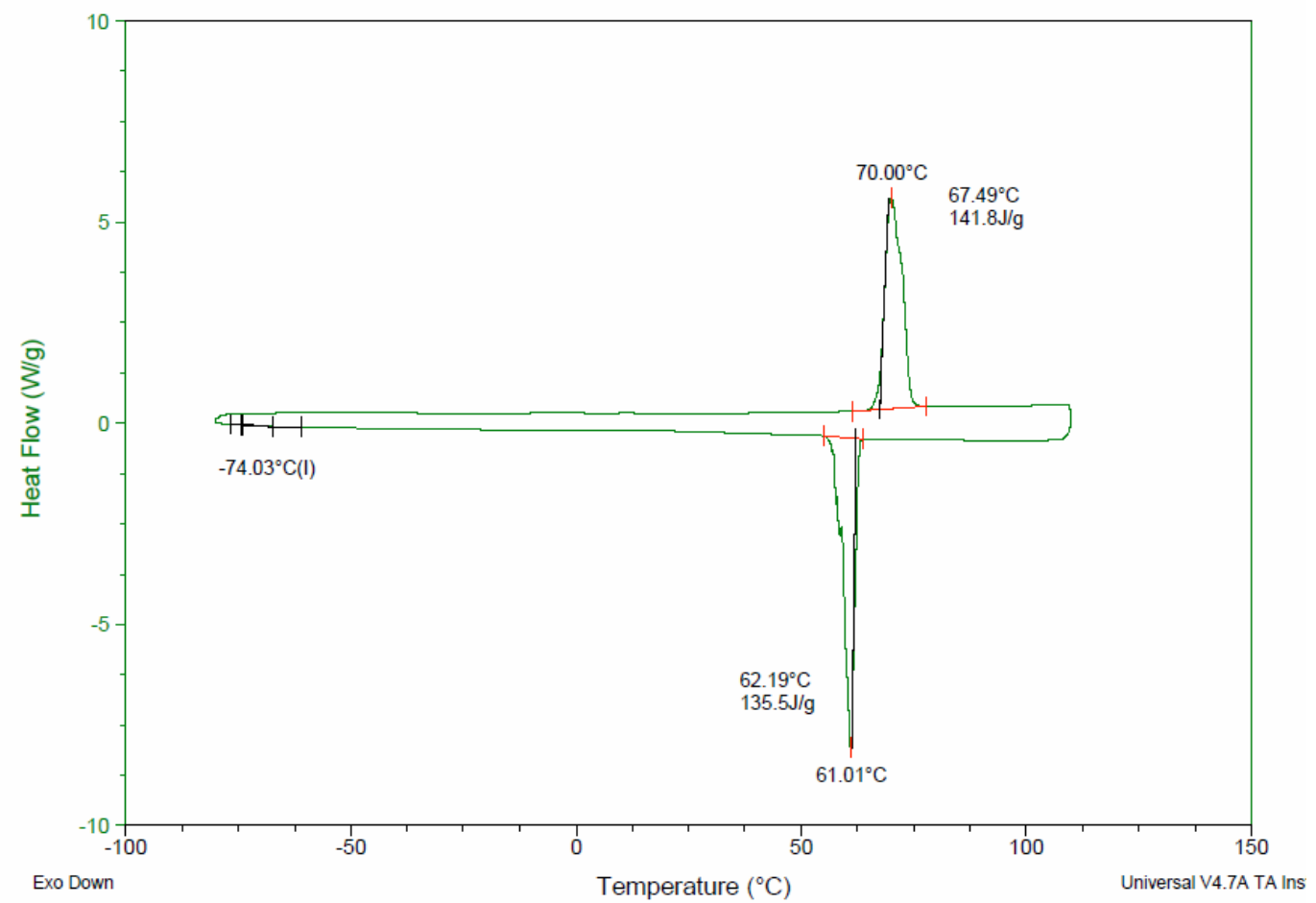

Figure S20. DSC Thermogram of polydecylene silicon acetal (Table S1, entry 4). 


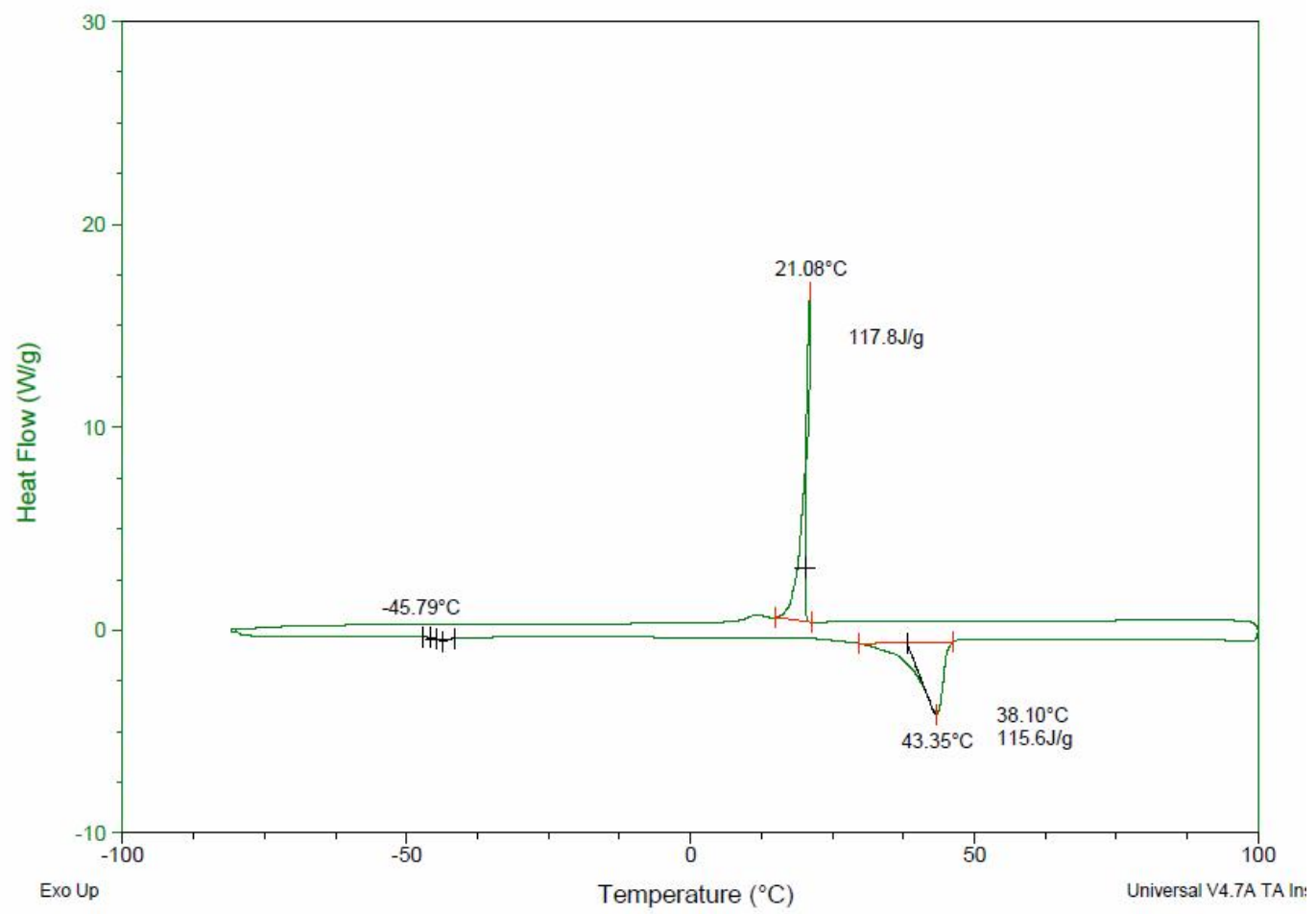

Figure S21. DSC Thermogram of polyhexylene silicon acetal (Table S1, entry 5).

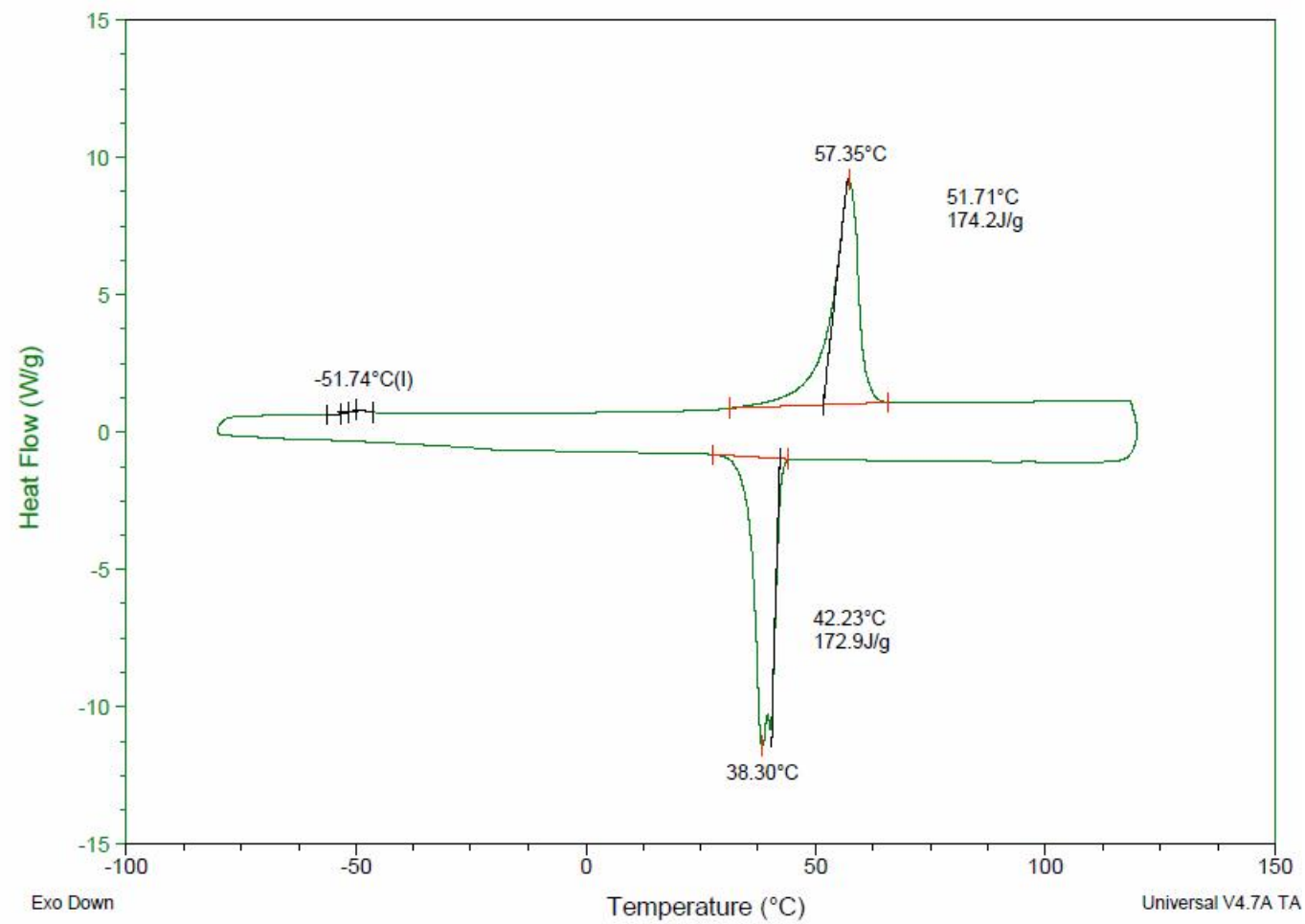

Figure S22. DSC Thermogram of polyoctlyne silicon acetal (Table S1, entry 6). 


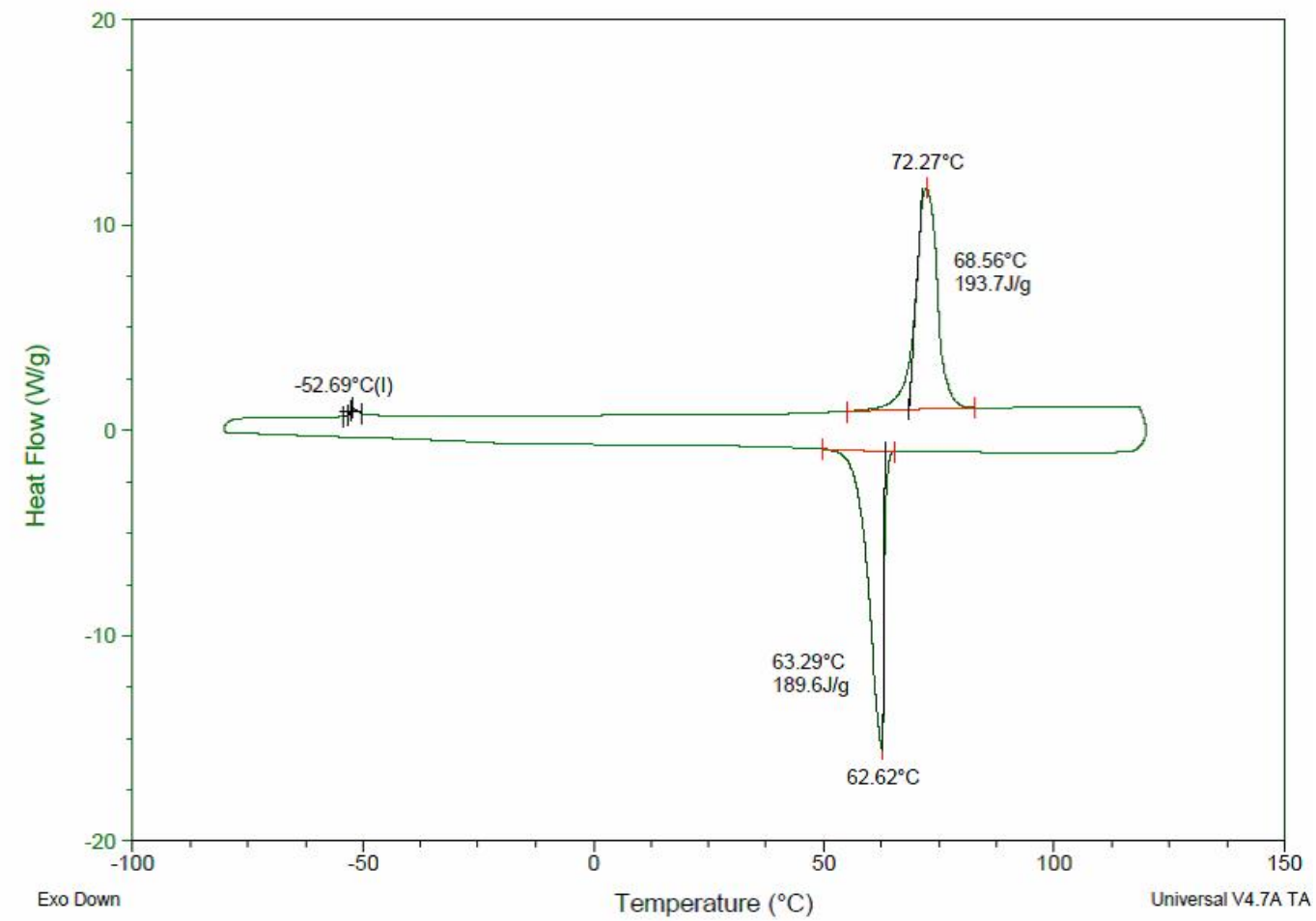

Figure S23. DSC Thermogram of polynonylene silicon acetal (Table S1, entry 7).

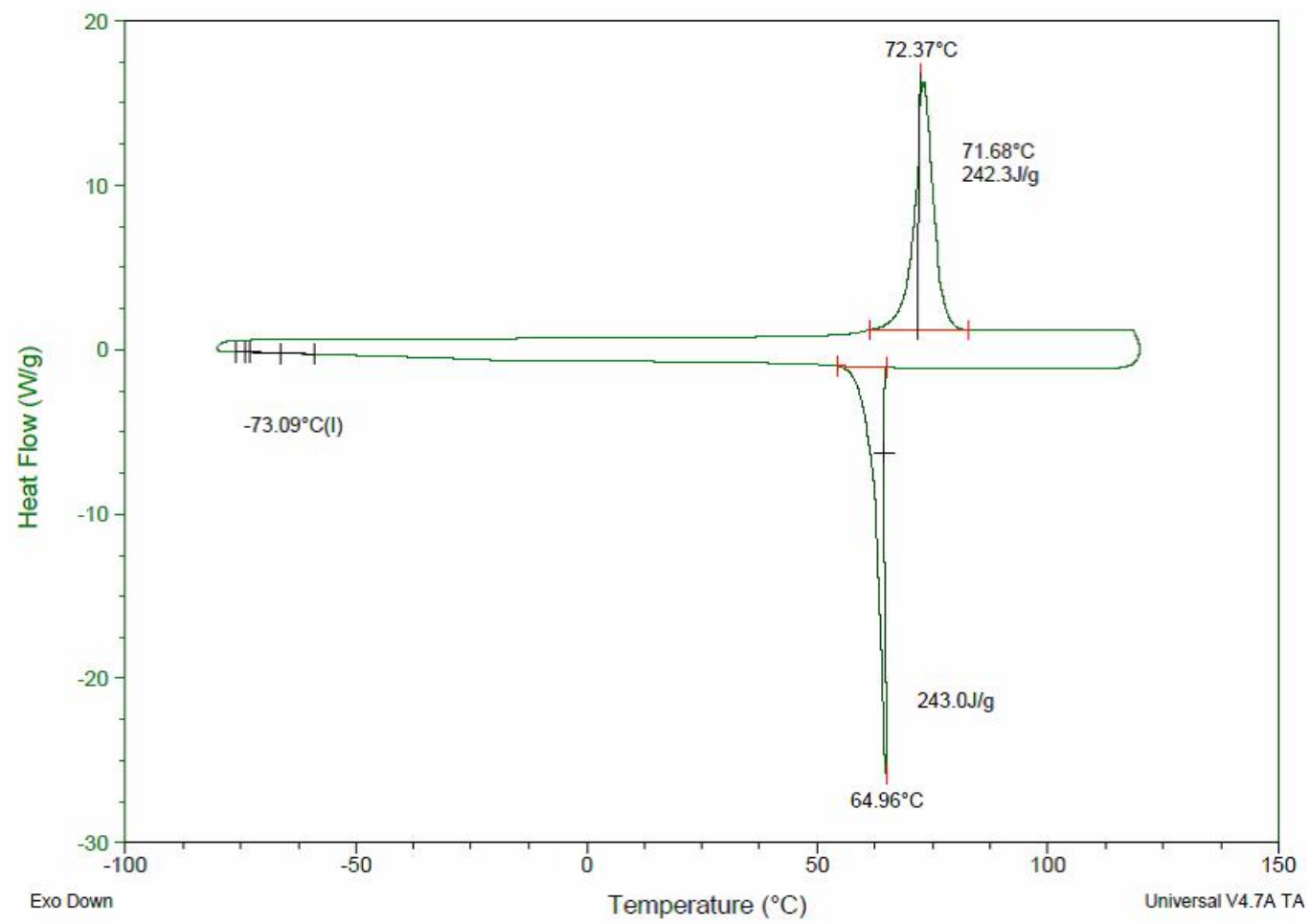

Figure S24. DSC Thermogram of polydecylene silicon acetal (Table S1, entry 8). 


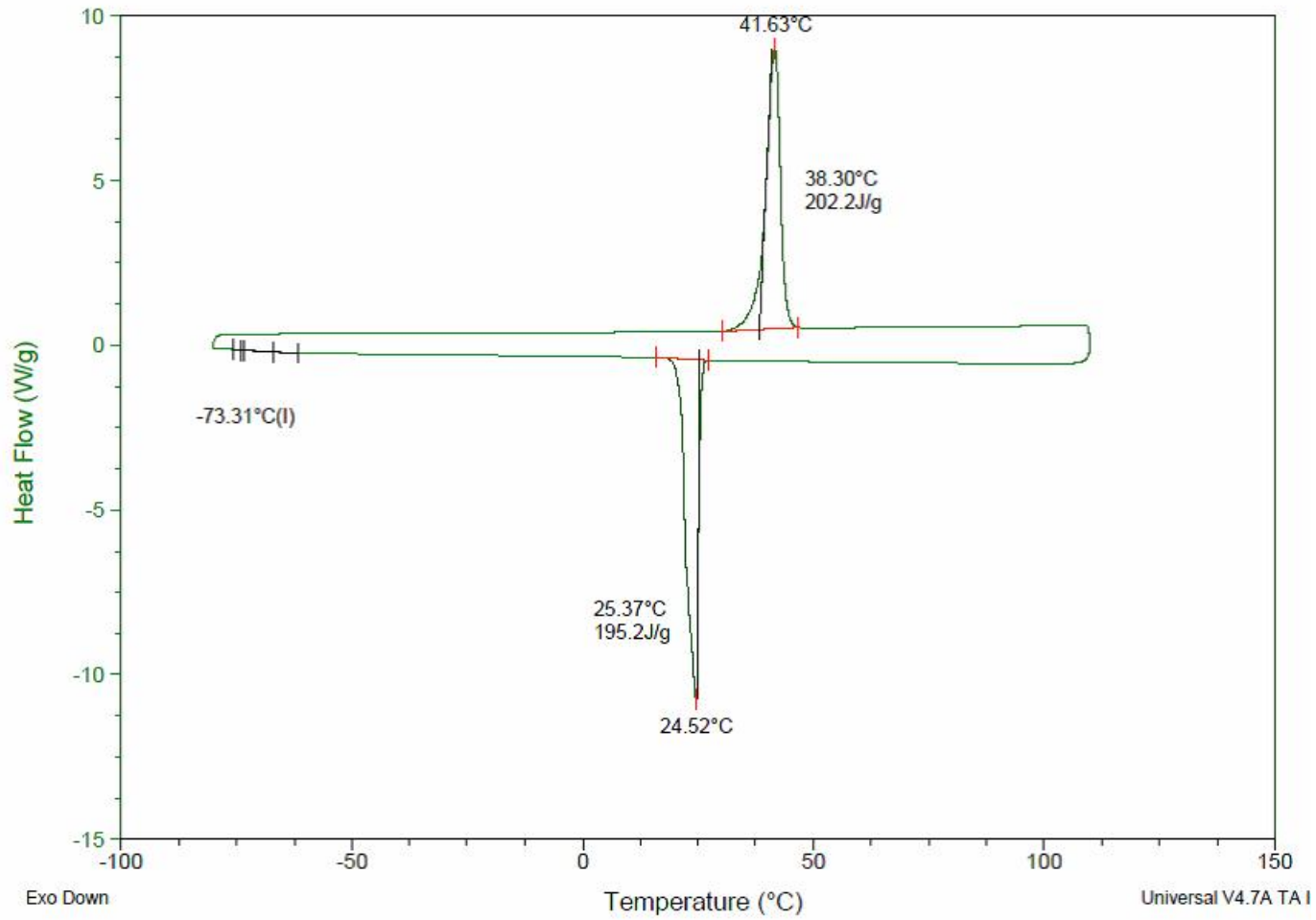

Figure S25. DSC Thermogram of polyhexylene silicon acetal (Table S1, entry 9).

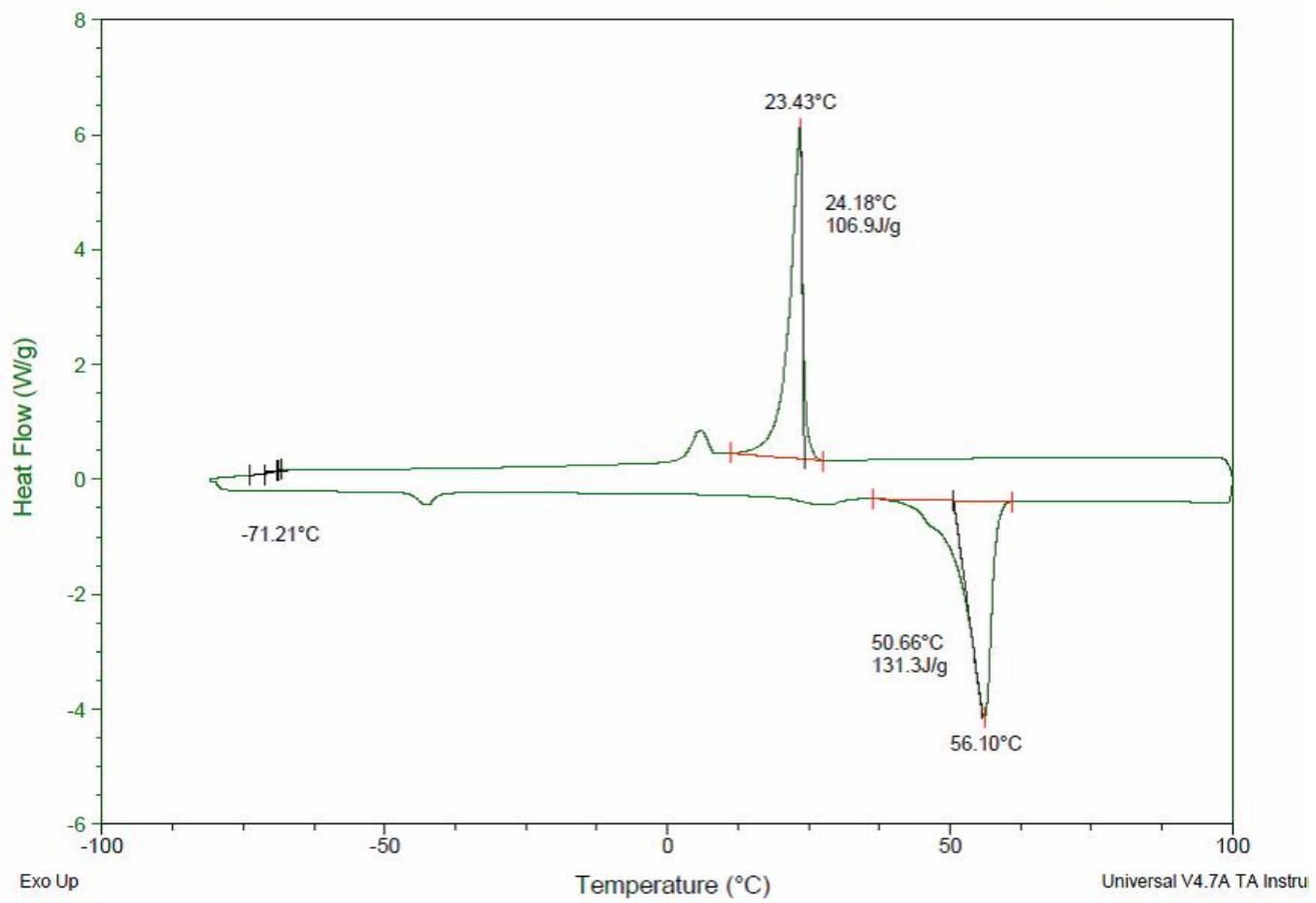

Figure S26. DSC Thermogram of polyoctlyne silicon acetal (Table S1, entry 10). 


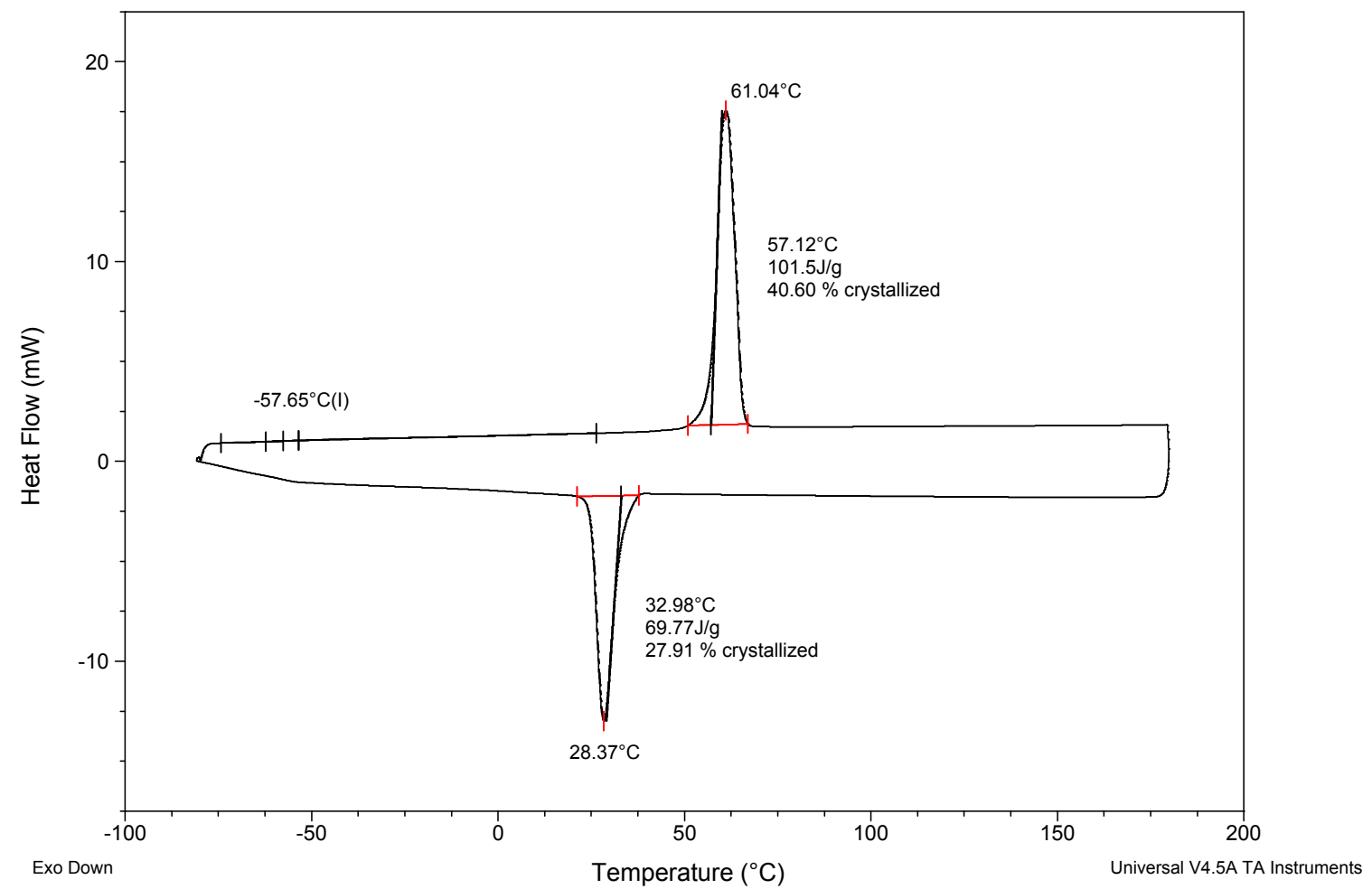

Figure S27. DSC Thermogram of polynonylene silicon acetal (Table S1, entry 11).

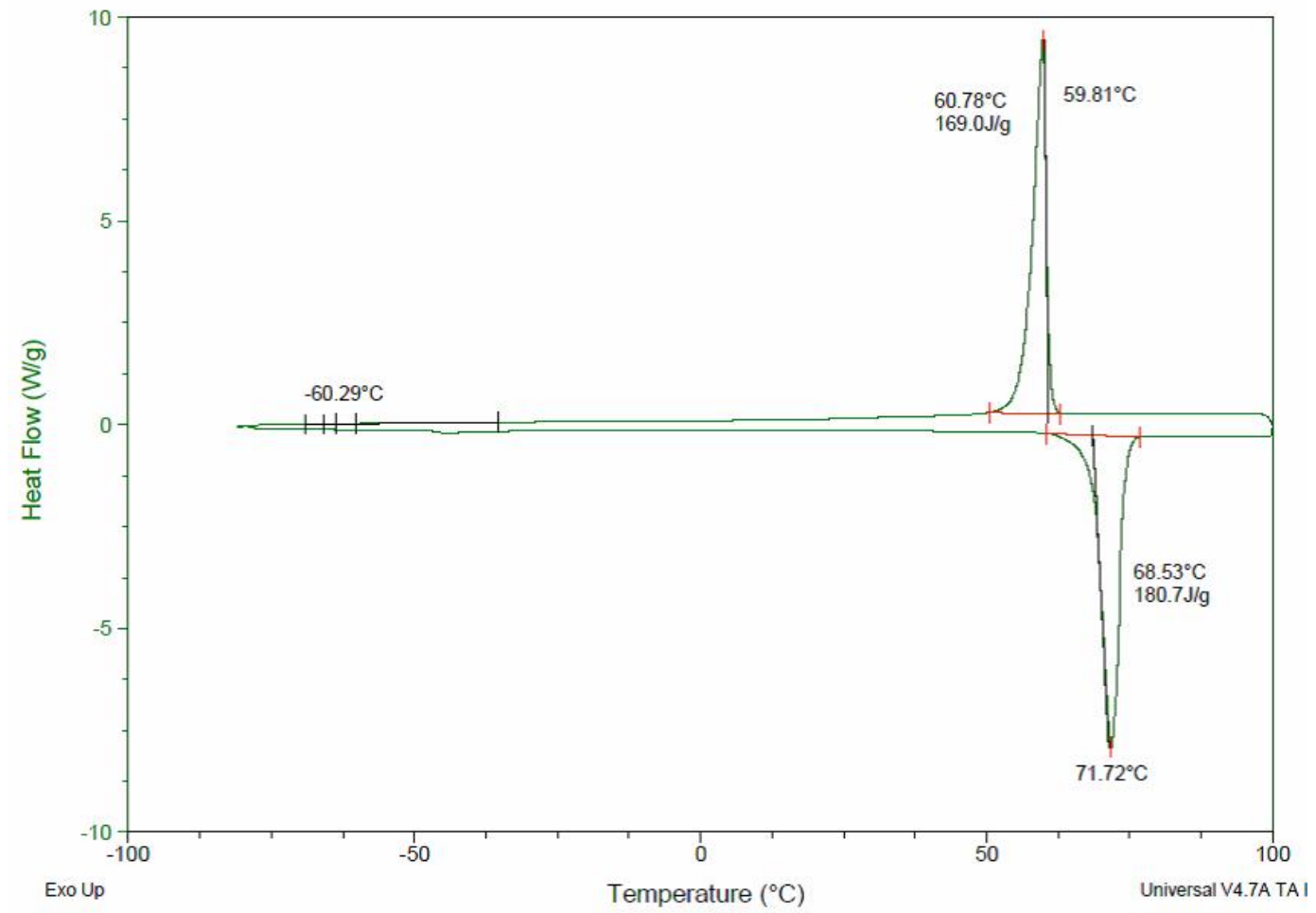

Figure S28. DSC Thermogram of polydecylene silicon acetal (Table S1, entry 12). 


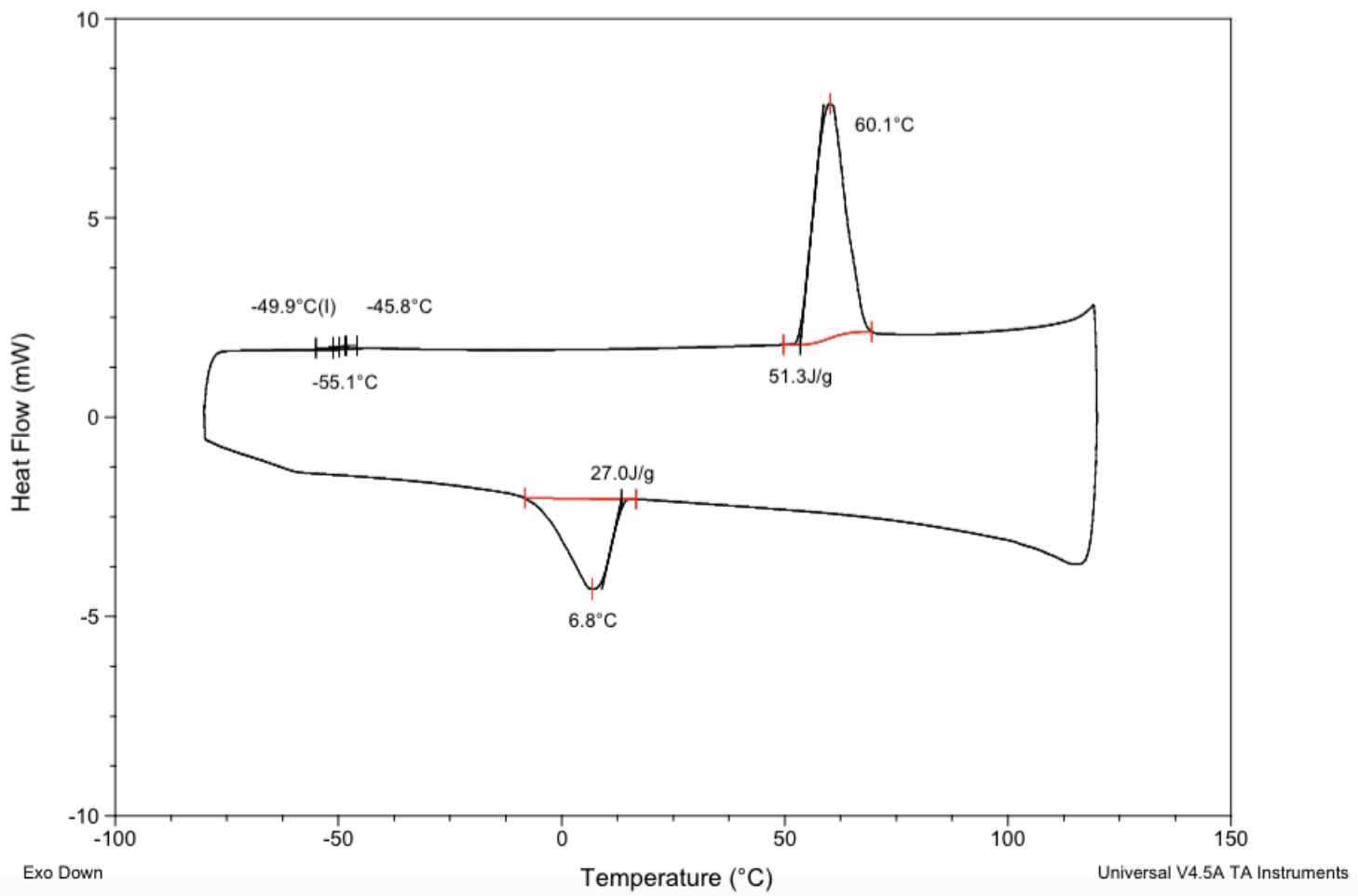

Figure S29. DSC Thermogram of polycamphoric silicon acetal (Table S1, entry 13).

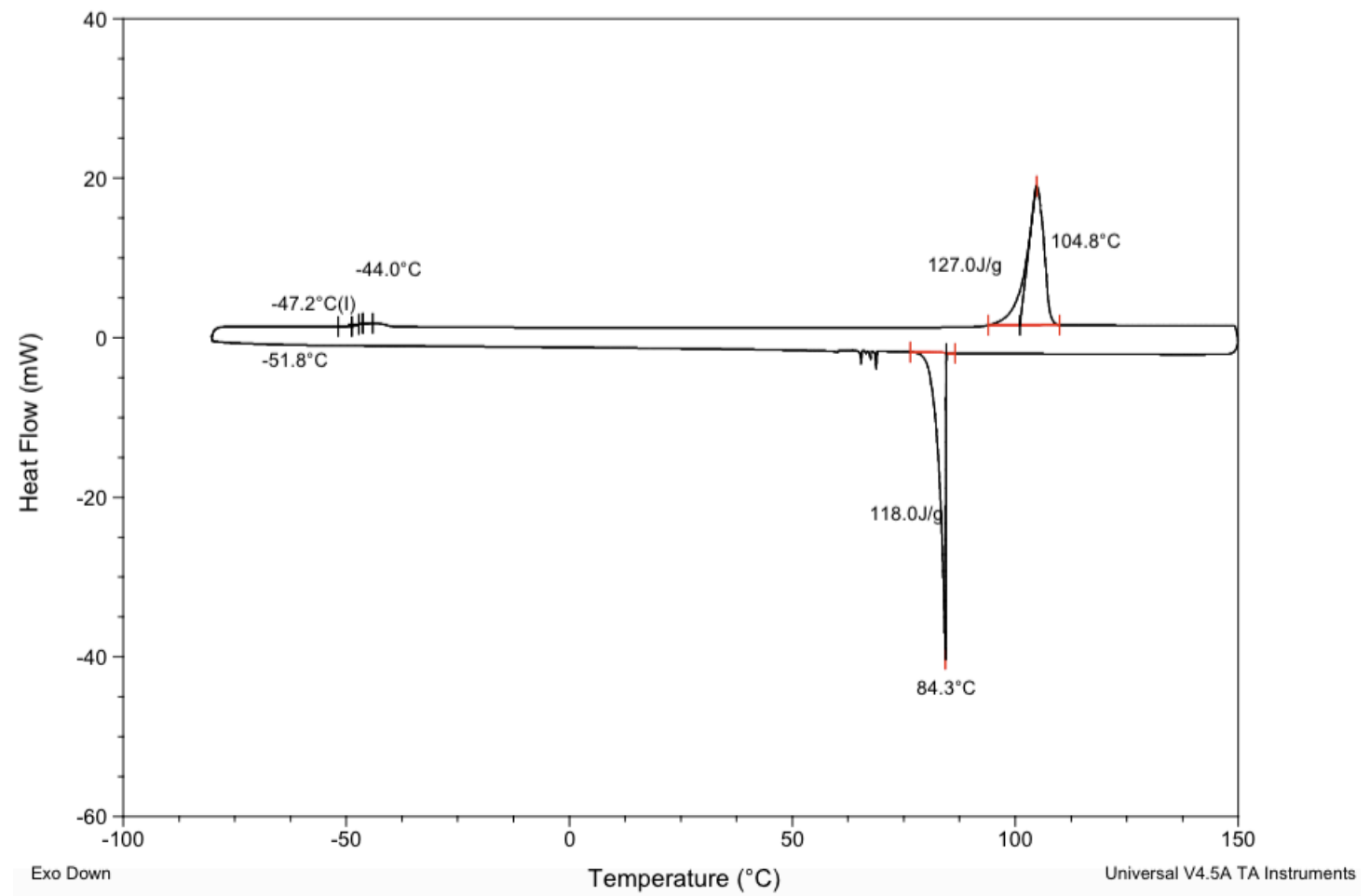

Figure S30. DSC Thermogram of polyethylene hydroquinoate silicon acetal (Table S1, entry 14). 


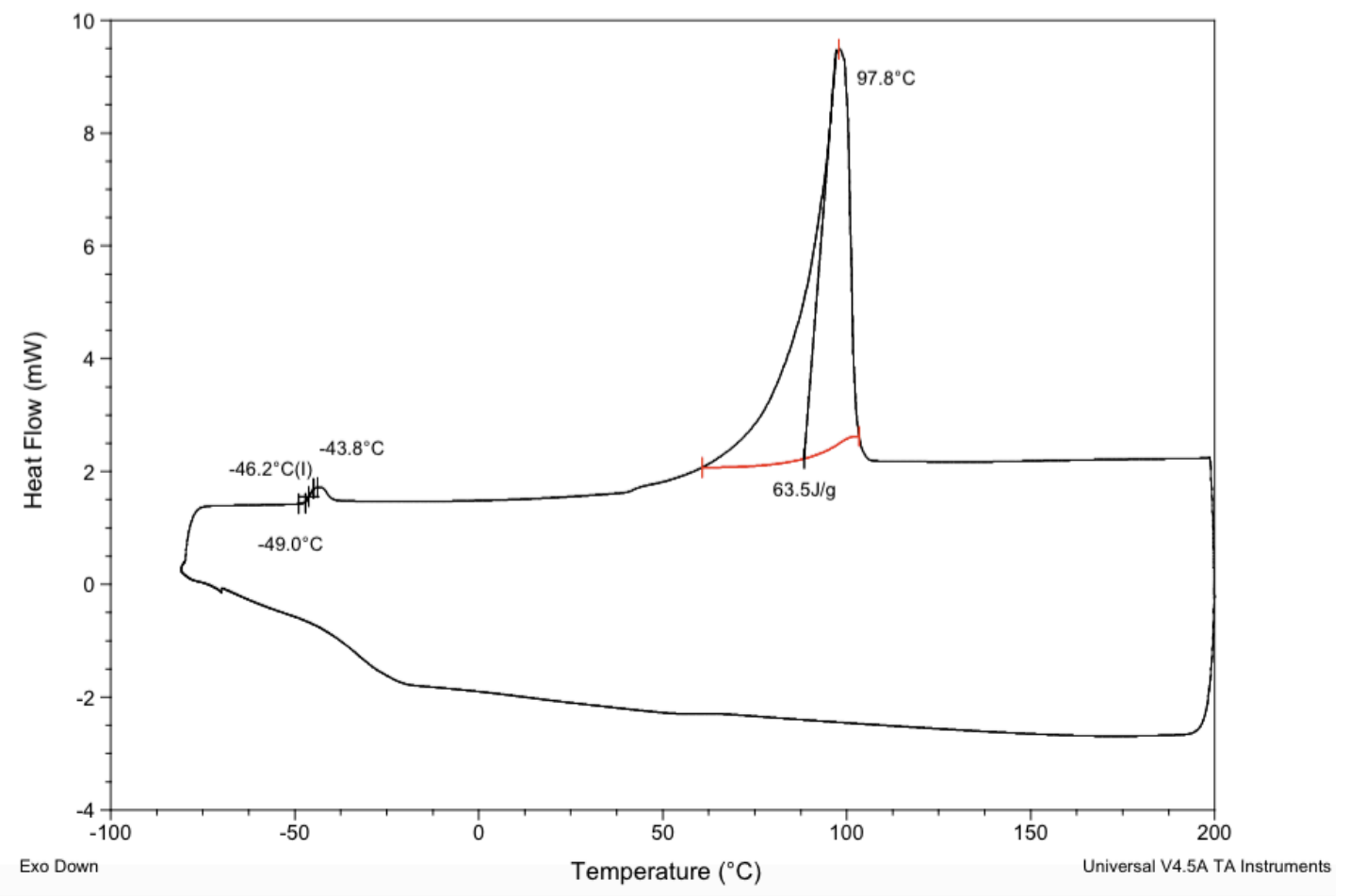

Figure S31. DSC Thermogram of polyethylene terephthalate silicon acetal (Table S1, entry 15).

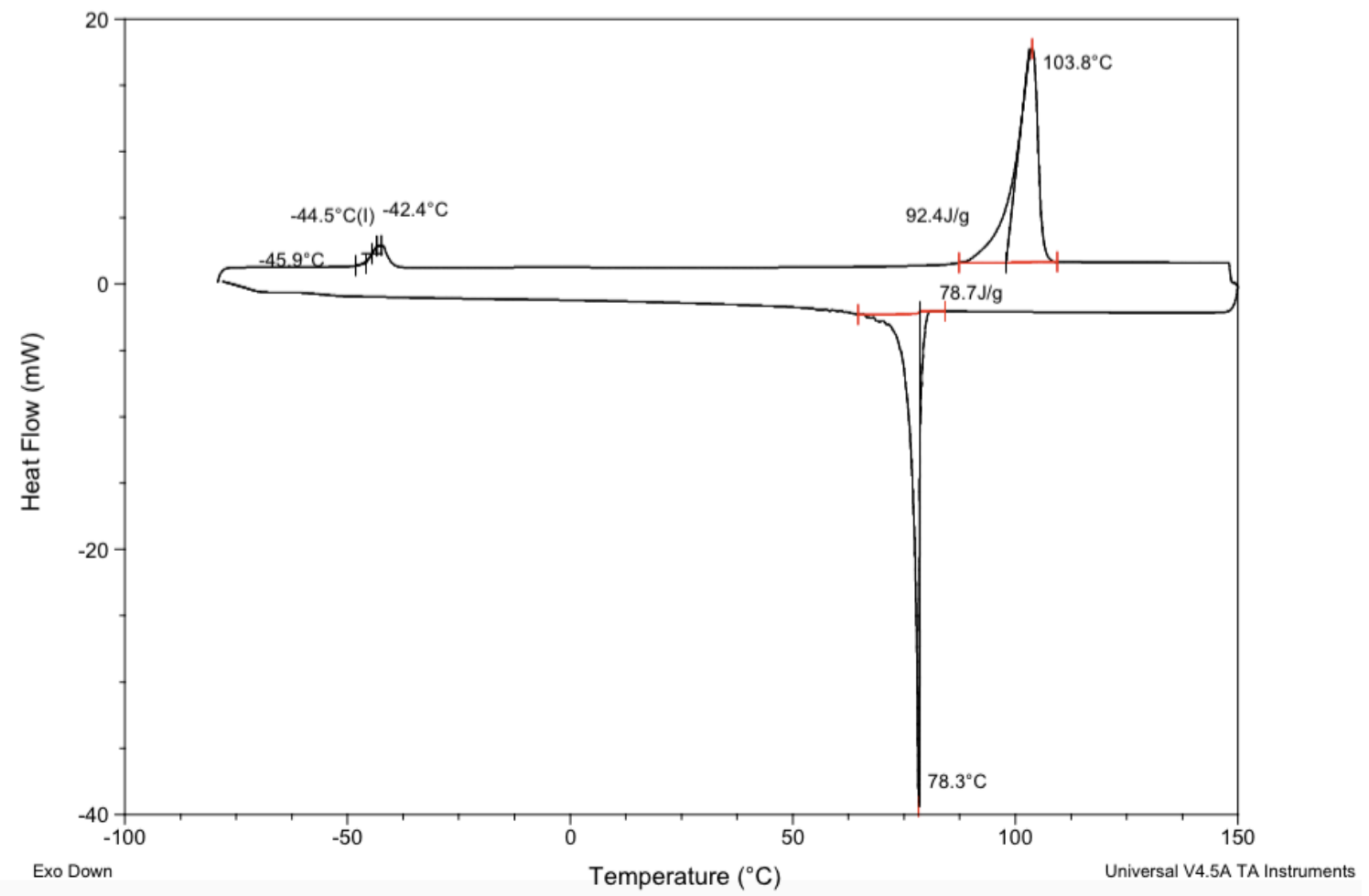

Figure S32. DSC Thermogram of polyethylene hydroquinoate silicon acetal (Table S1, entry 16). 


\section{Thermogravimetric Analysis (TGA) Thermograms}

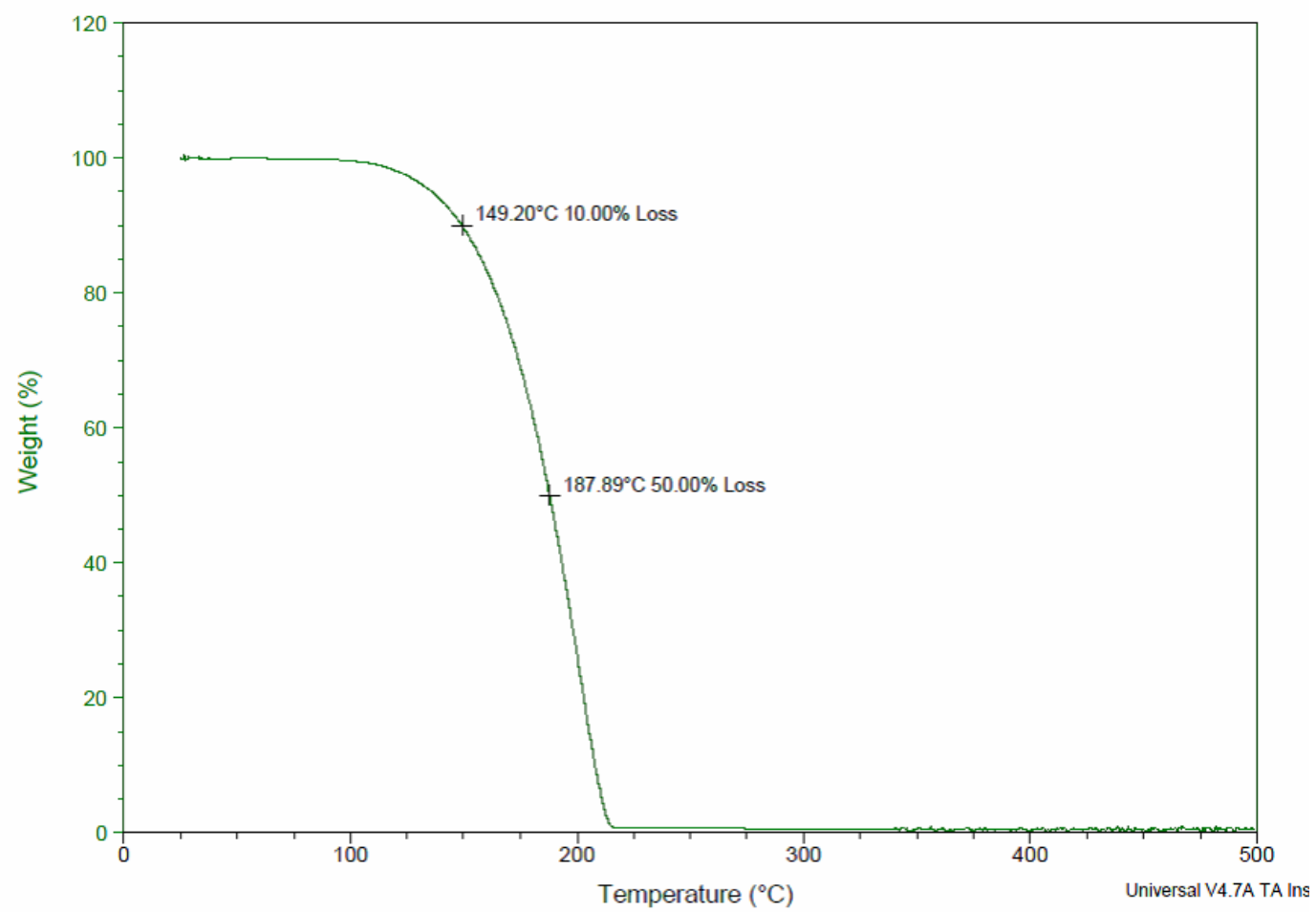

Figure S33. TGA Thermogram of polyheptylene silicon acetal (Table S1, entry 1).

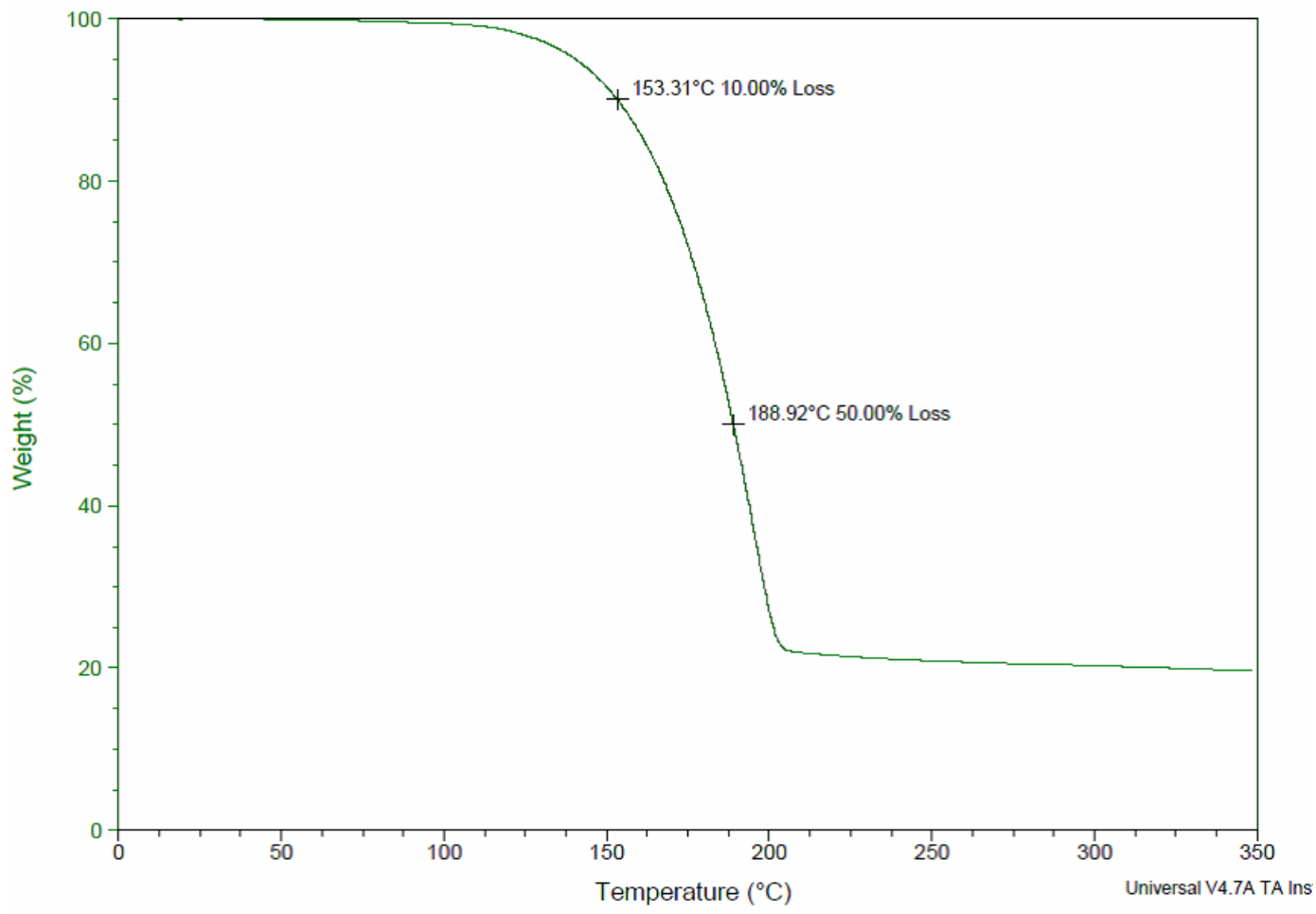

Figure S34. TGA Thermogram of polyoctlyne silicon acetal (Table S1, entry 2). 


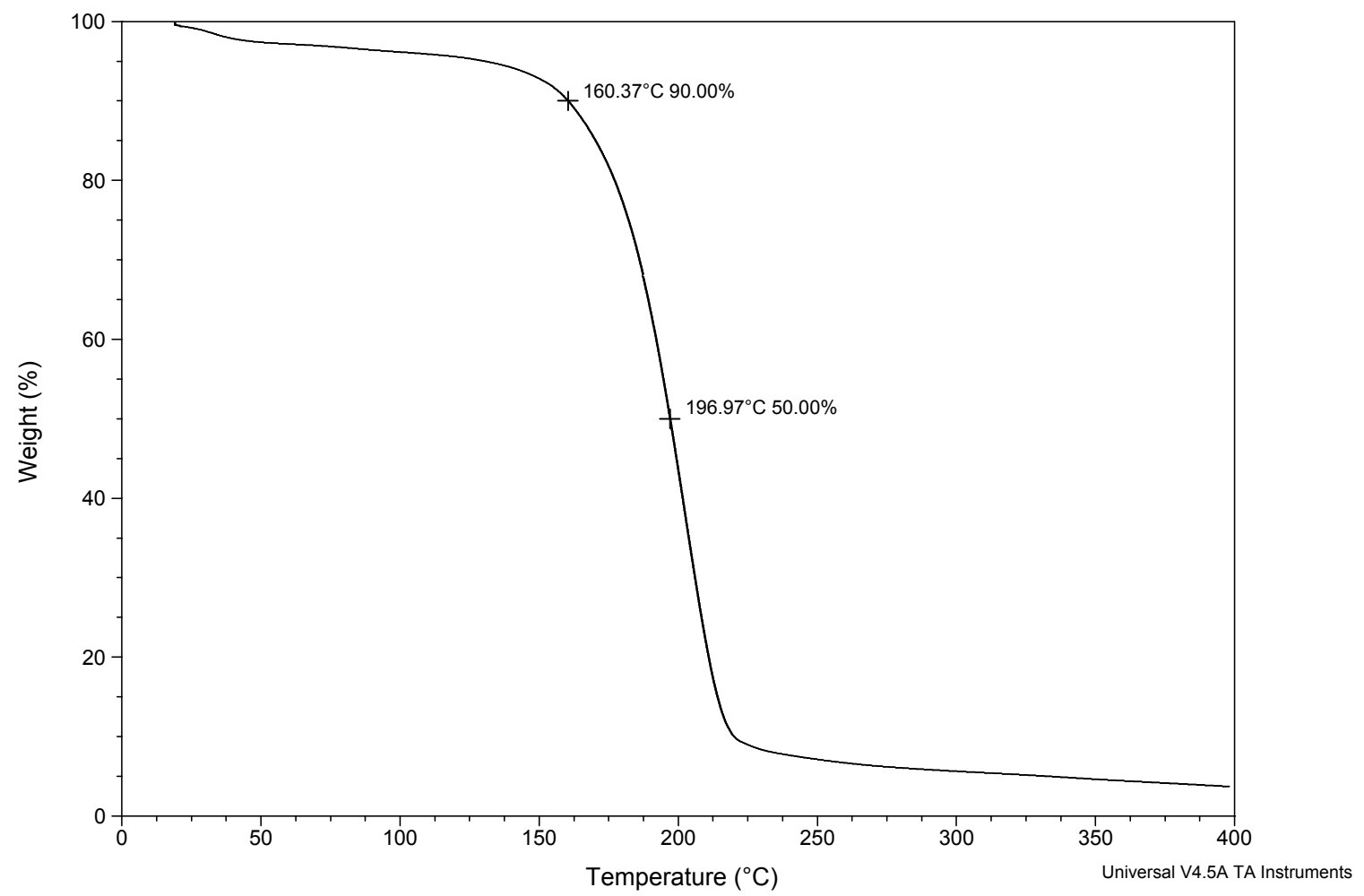

Figure S35. TGA Thermogram of polynonylene silicon acetal (Table S1, entry 3).

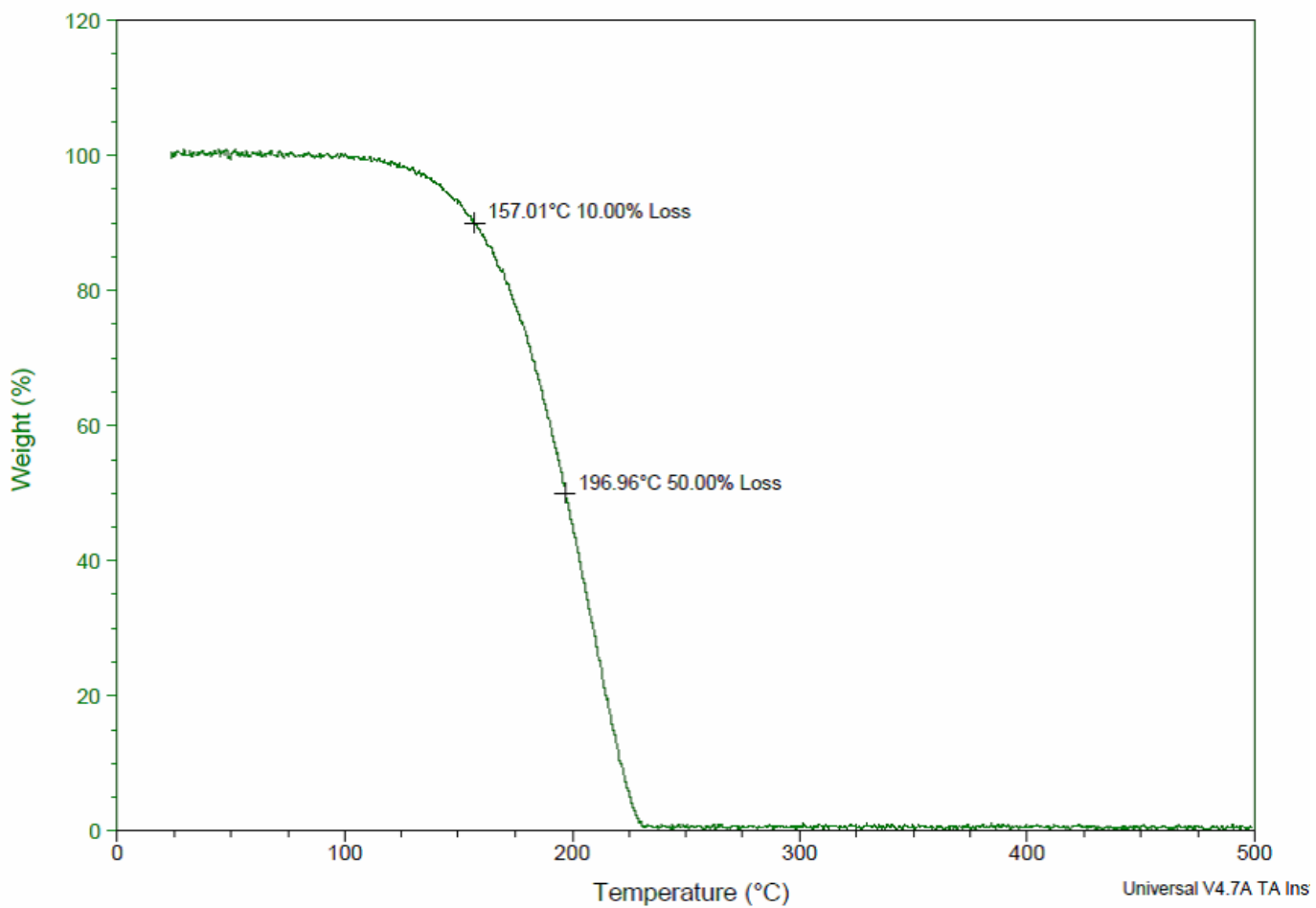

Figure S36. TGA Thermogram of polydecylene silicon acetal (Table S1, entry 4). 


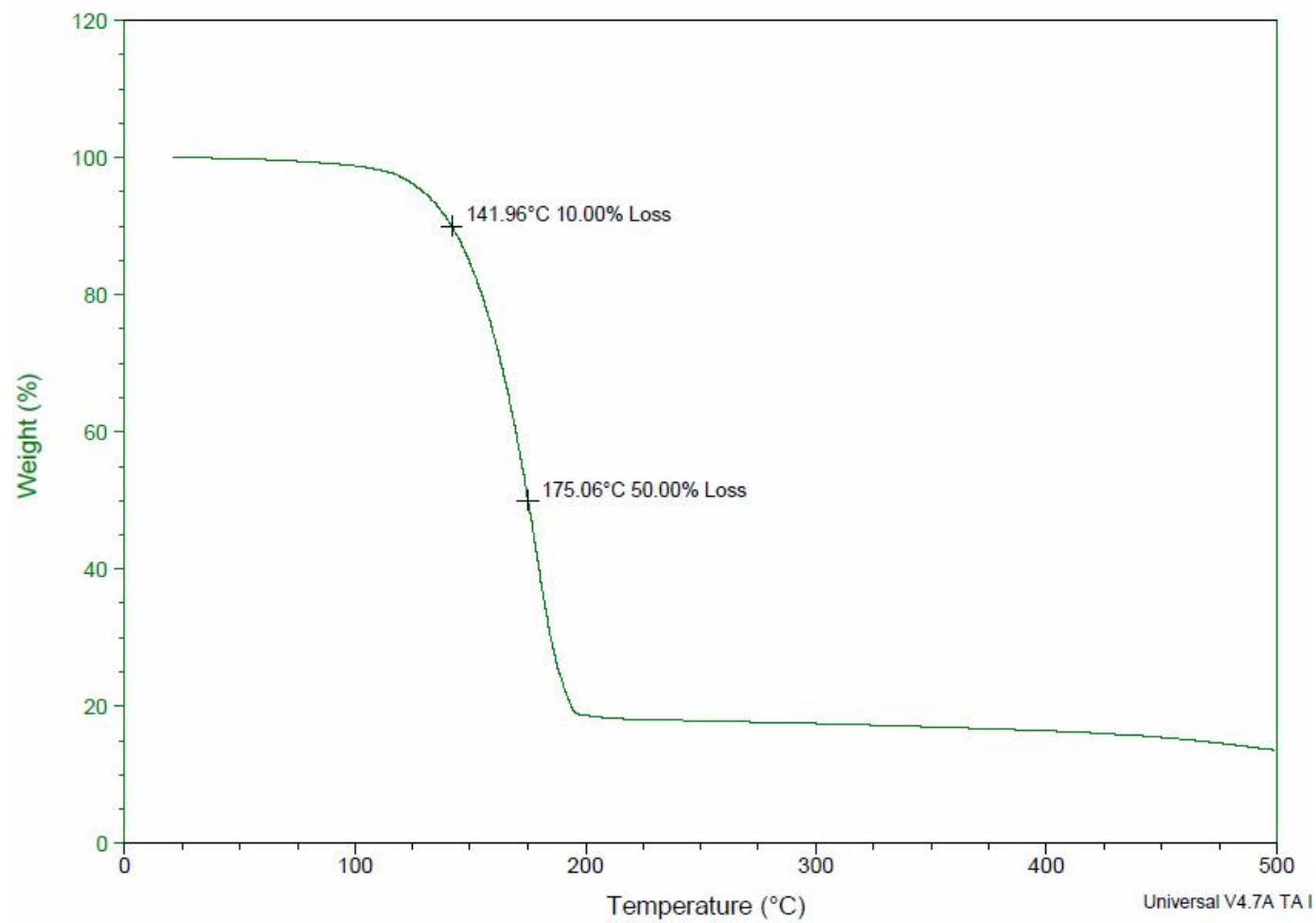

Figure S37. TGA Thermogram of polyhexylene silicon acetal (Table S1, entry 5).

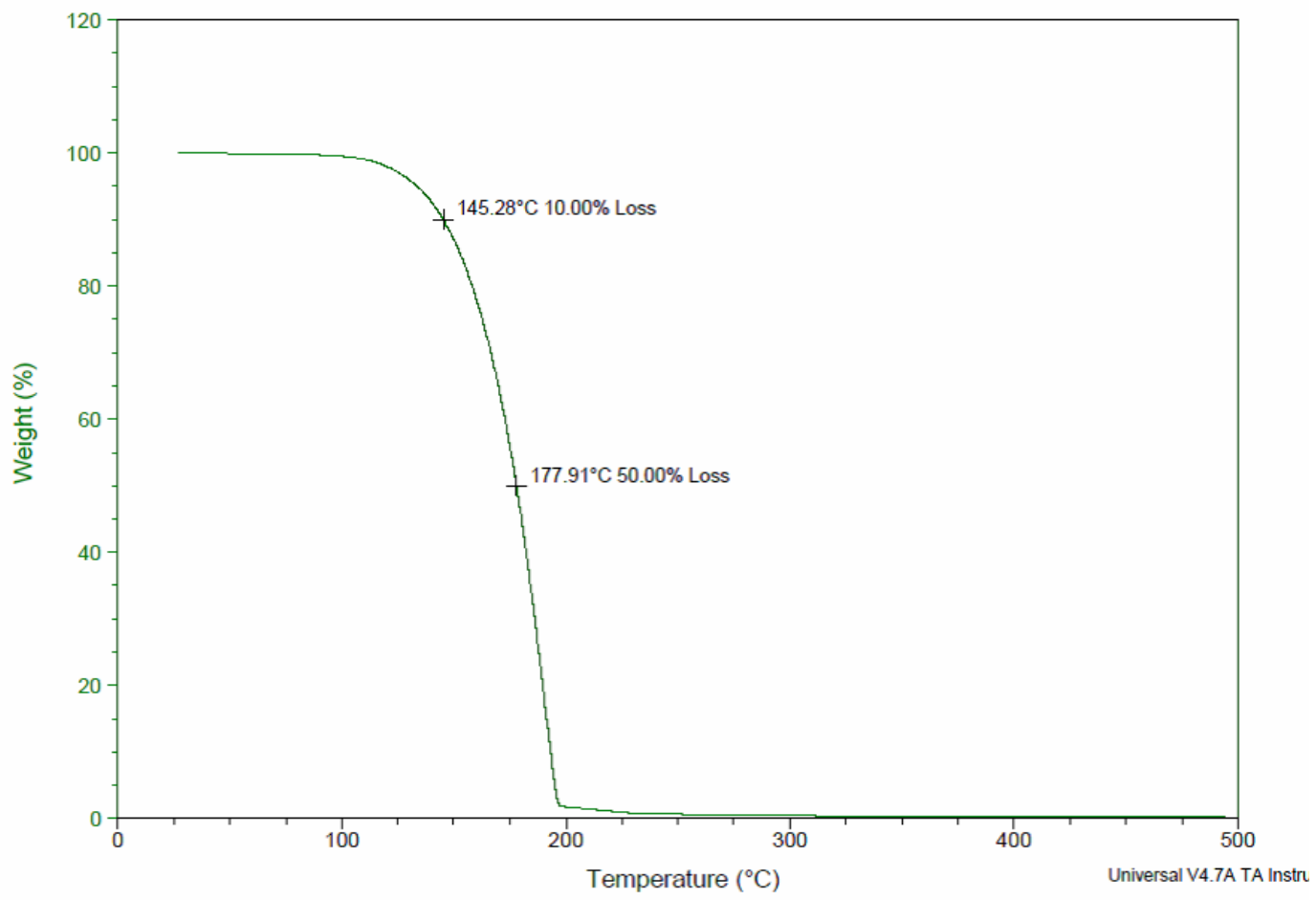

Figure S38. TGA Thermogram of polyoctylene silicon acetal (Table S1, entry 6). 


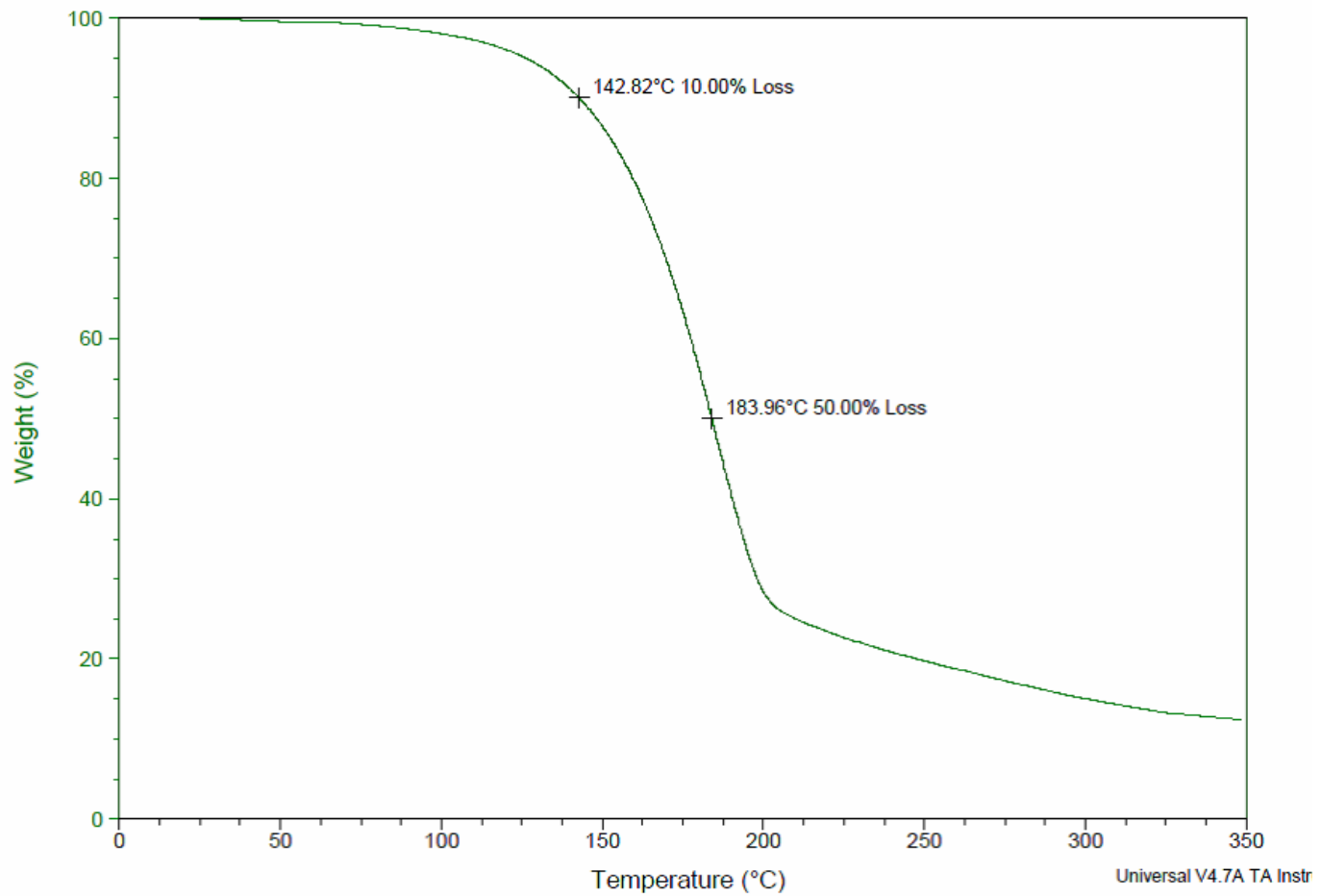

Figure S39. TGA Thermogram of polynonylene silicon acetal (Table S1, entry 7).

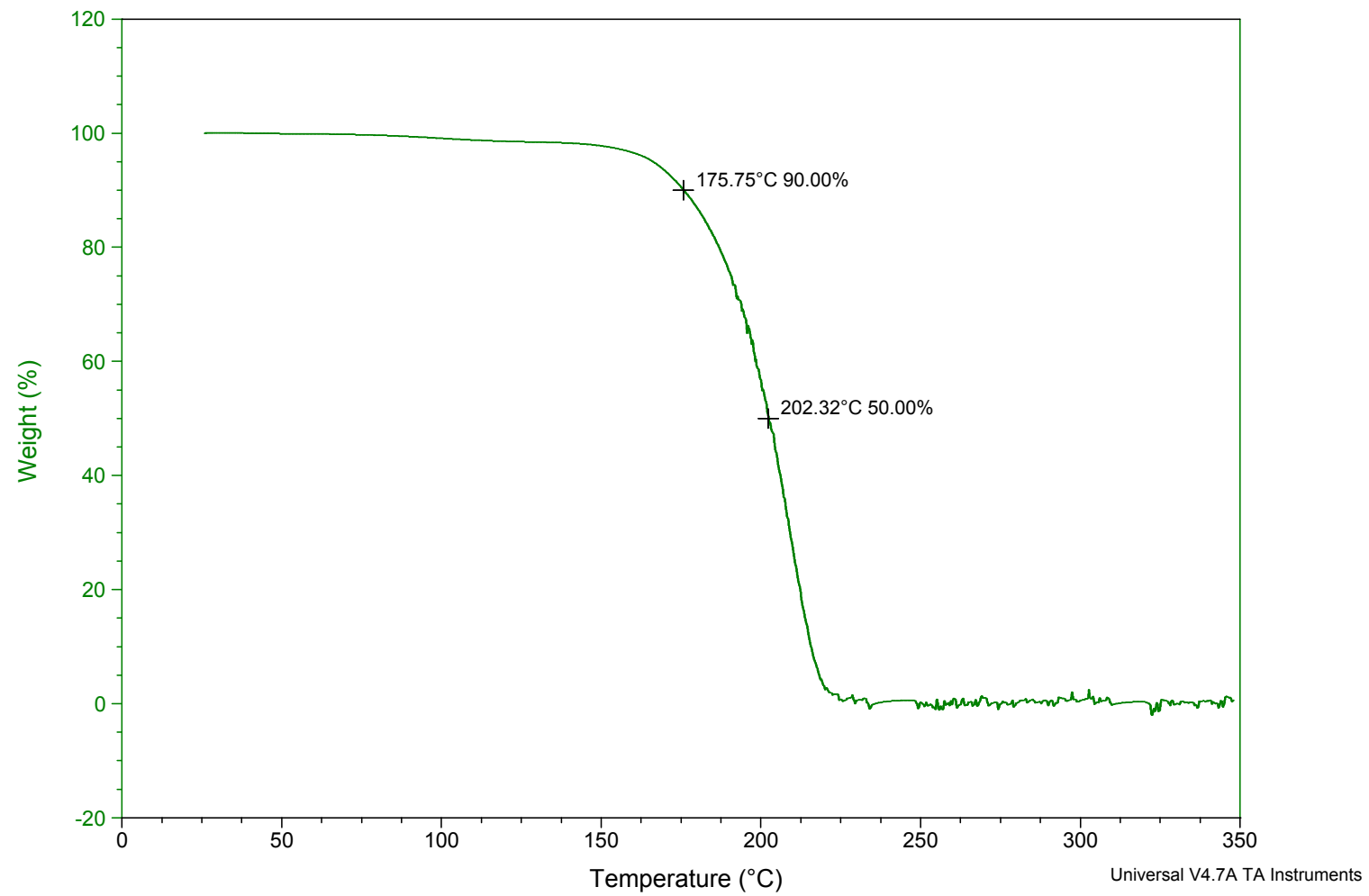

Figure S40. TGA Thermogram of polydecylene silicon acetal (Table S1, entry 8). 


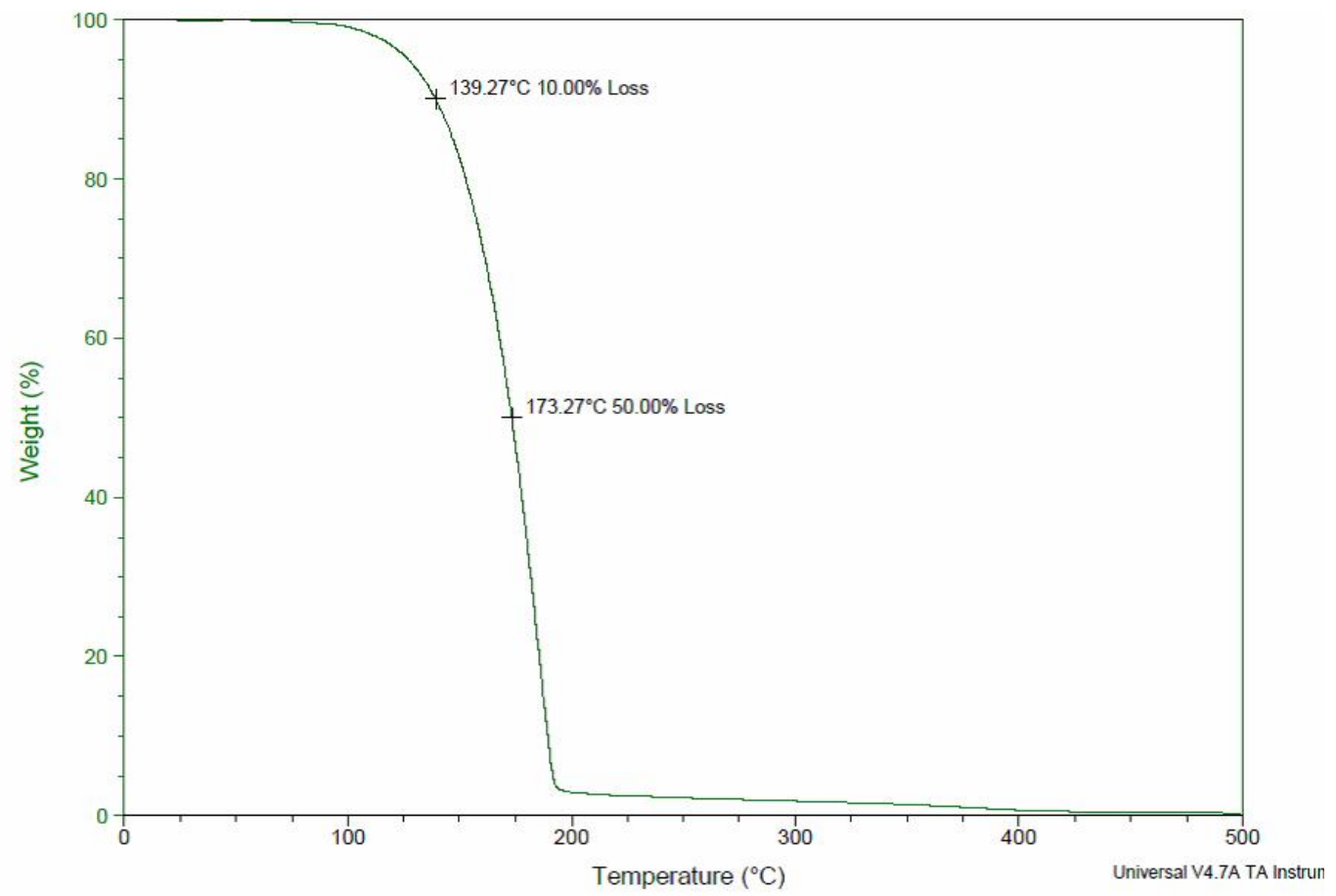

Figure S41. TGA Thermogram of polyhexylene silicon acetal (Table S1, entry 9).

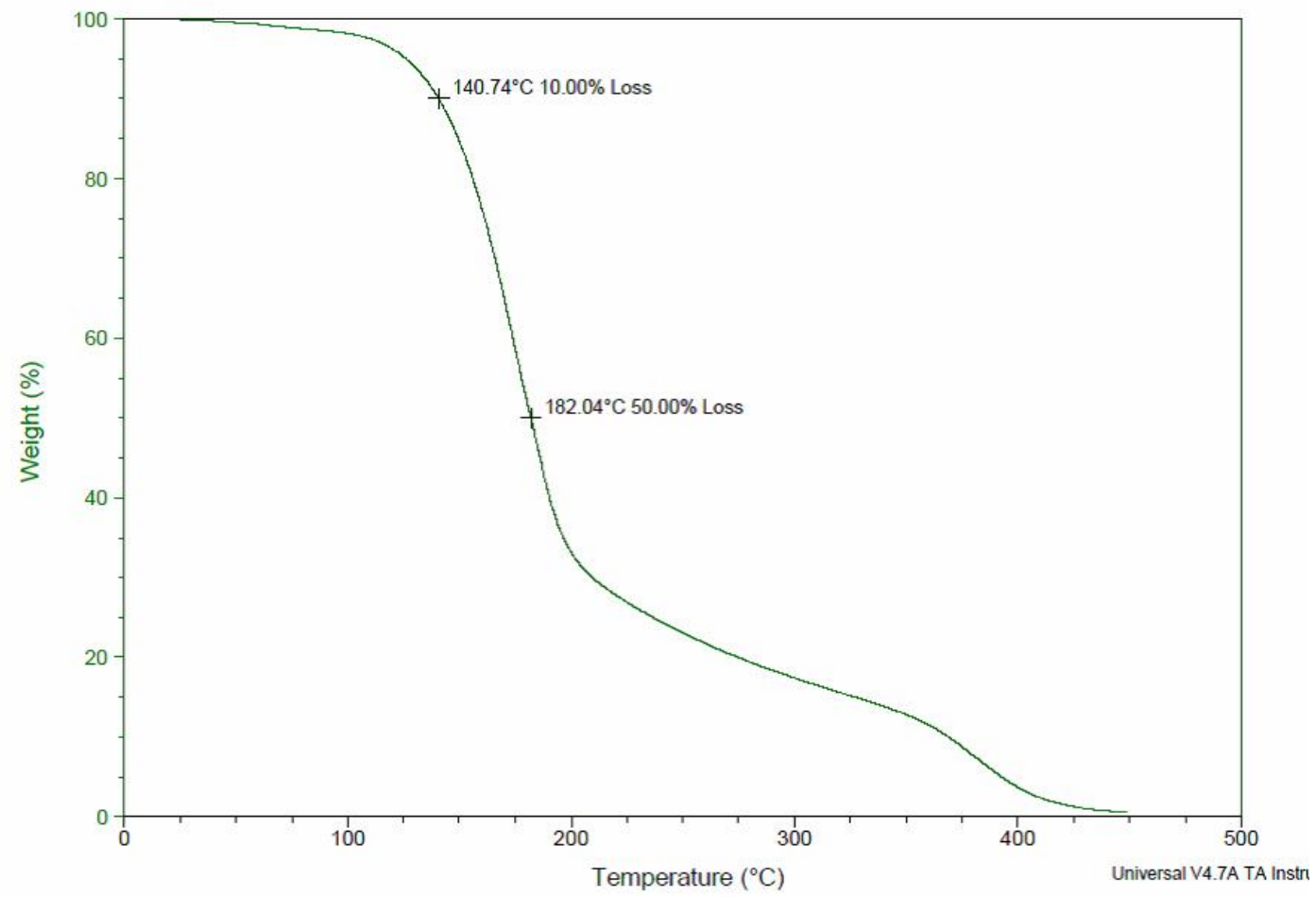

Figure S42. TGA Thermogram of polyoctylene silicon acetal (Table S1, Entry 10). 


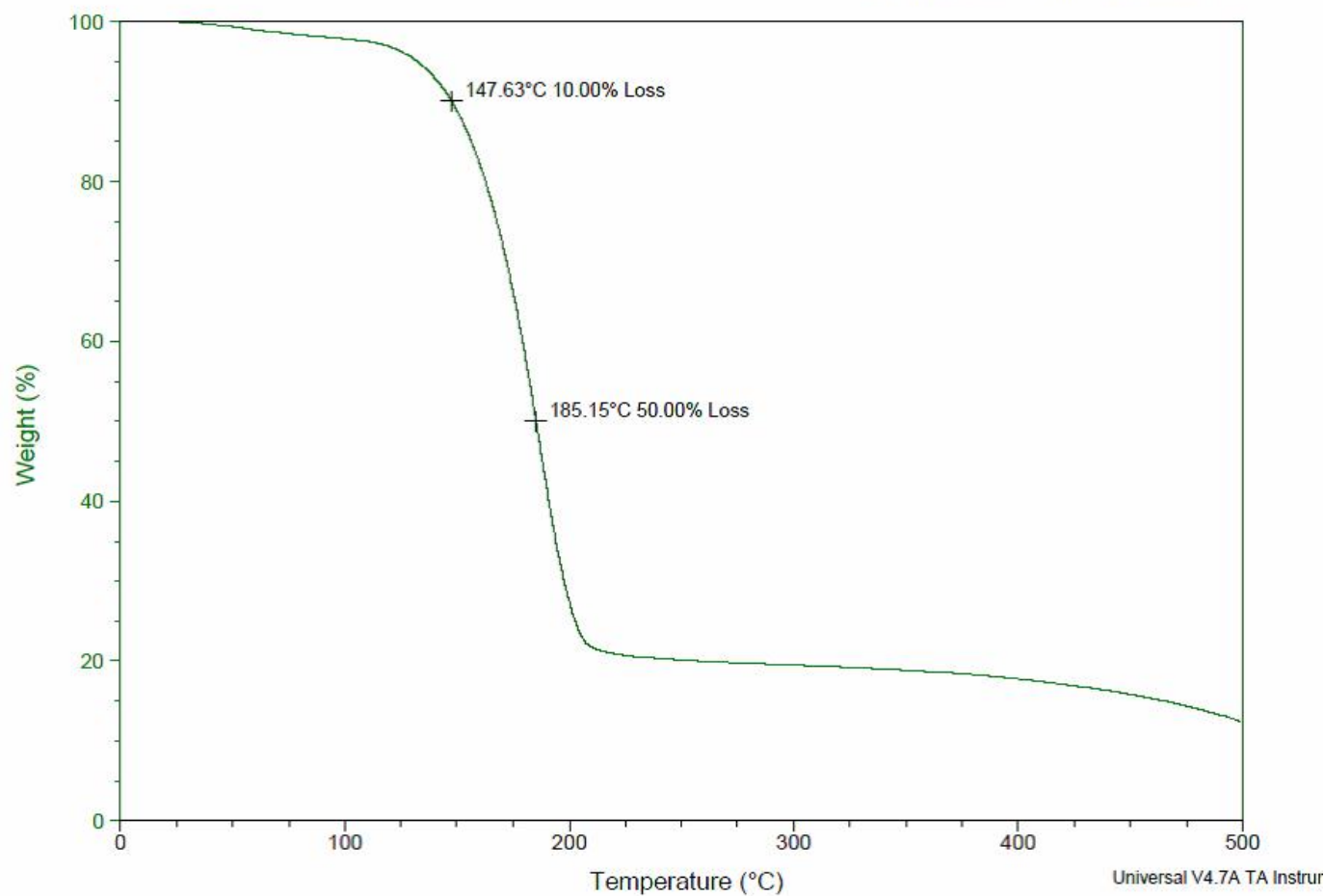

Figure S43. TGA Thermogram of polynonylene silicon acetal (Table S1, entry 11).

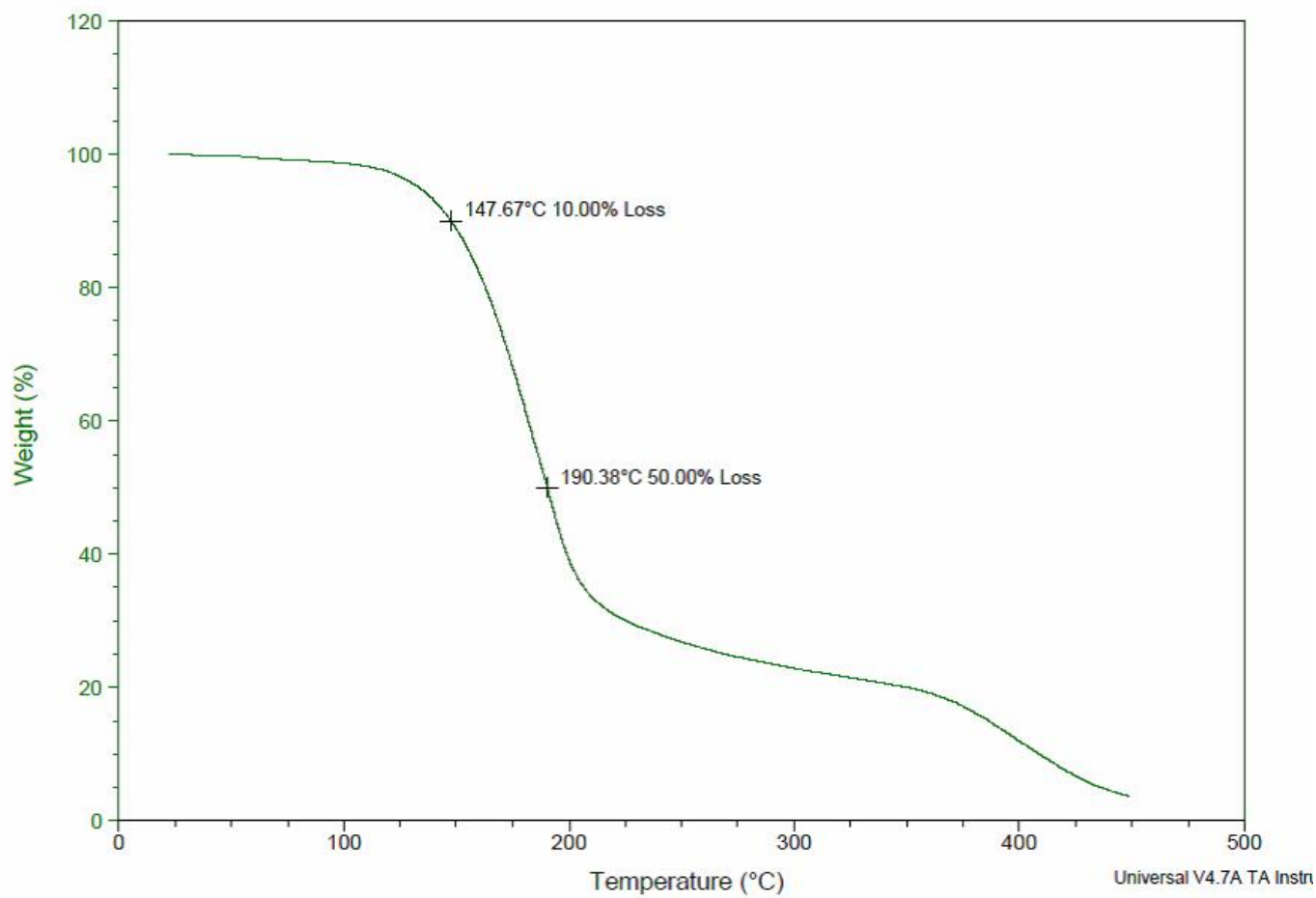

Figure S44. TGA Thermogram of polydecylene silicon acetal (Table S1, entry 12). 


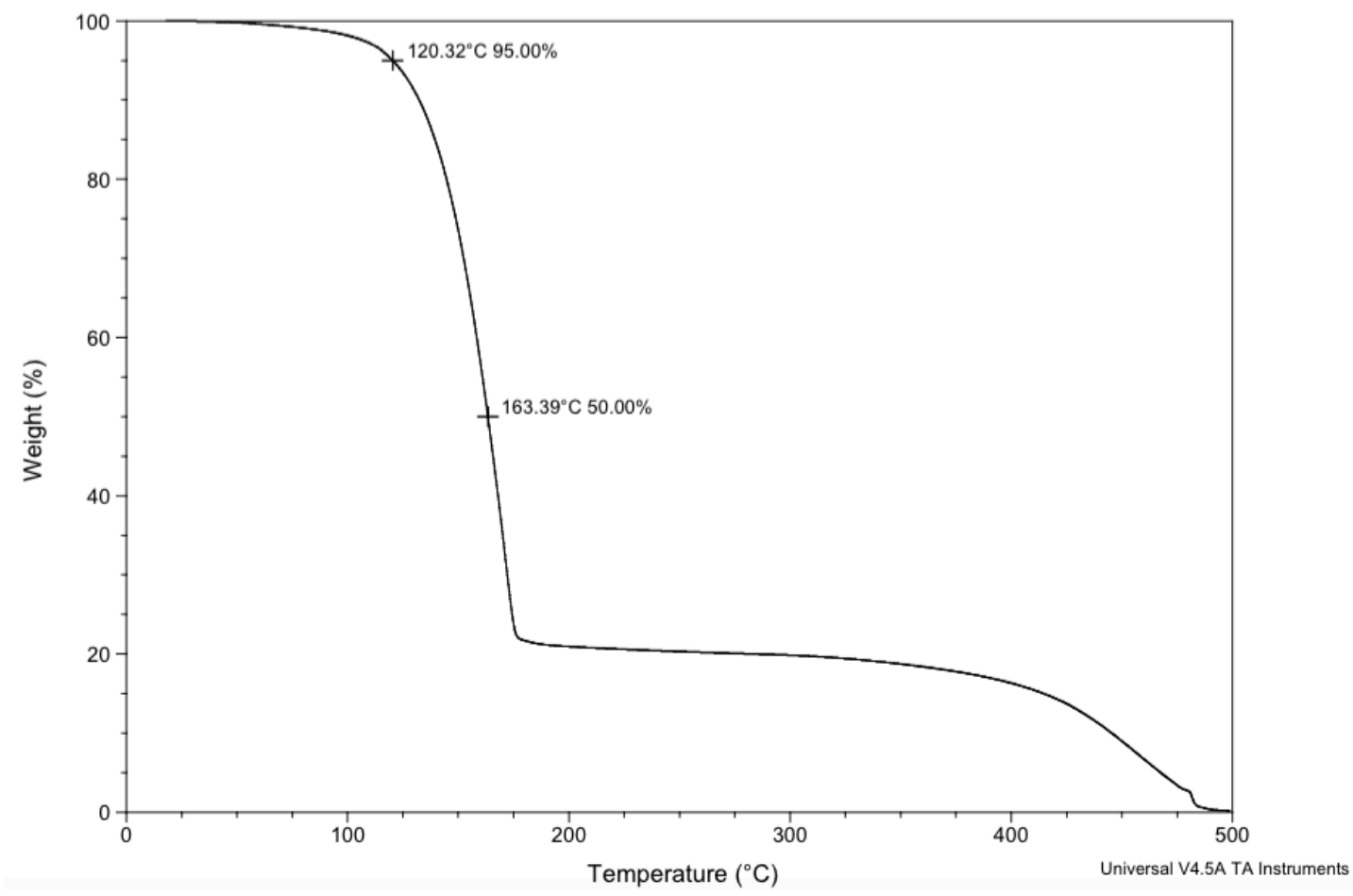

Figure S45. TGA thermogram of polycamphoric silicon acetal (Table S1, Entry 13).

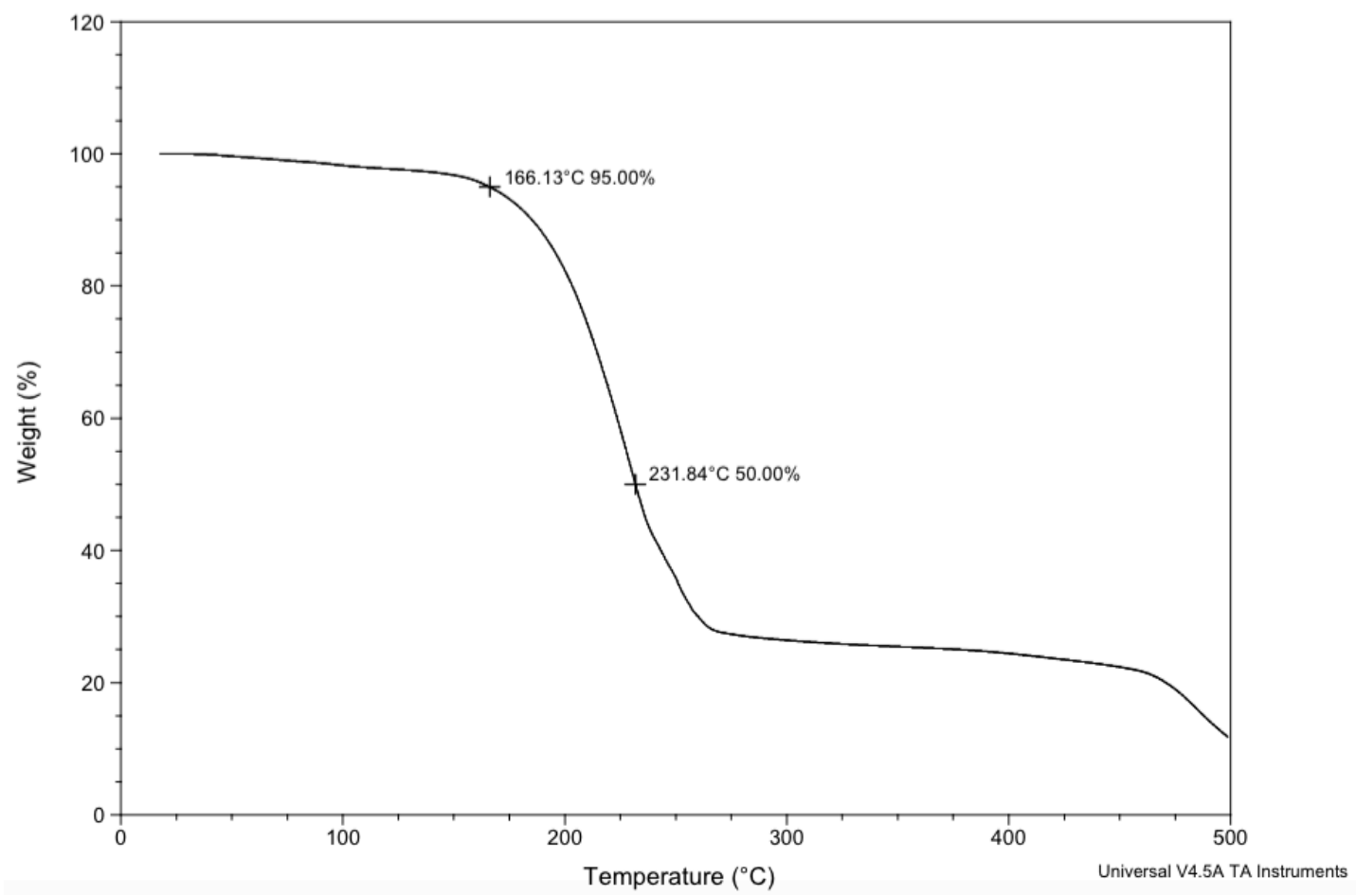

Figure S46. TGA Thermogram of polyethylene hydroquinoate silicon acetal (Table S1, Entry 14). 


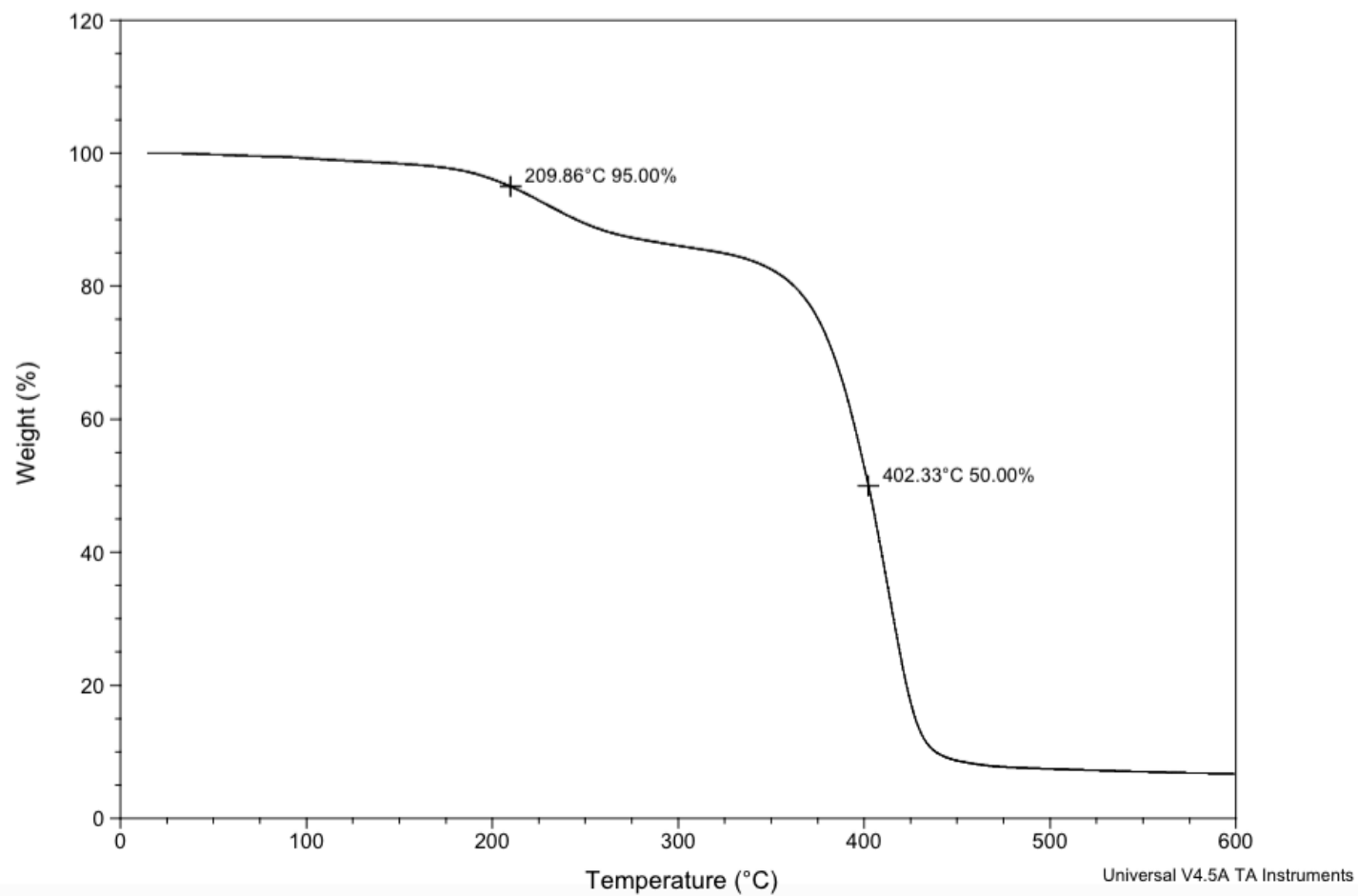

Figure S47. TGA Thermogram of polyethylene terephthalate silicon acetal (Table S1, Entry 15).

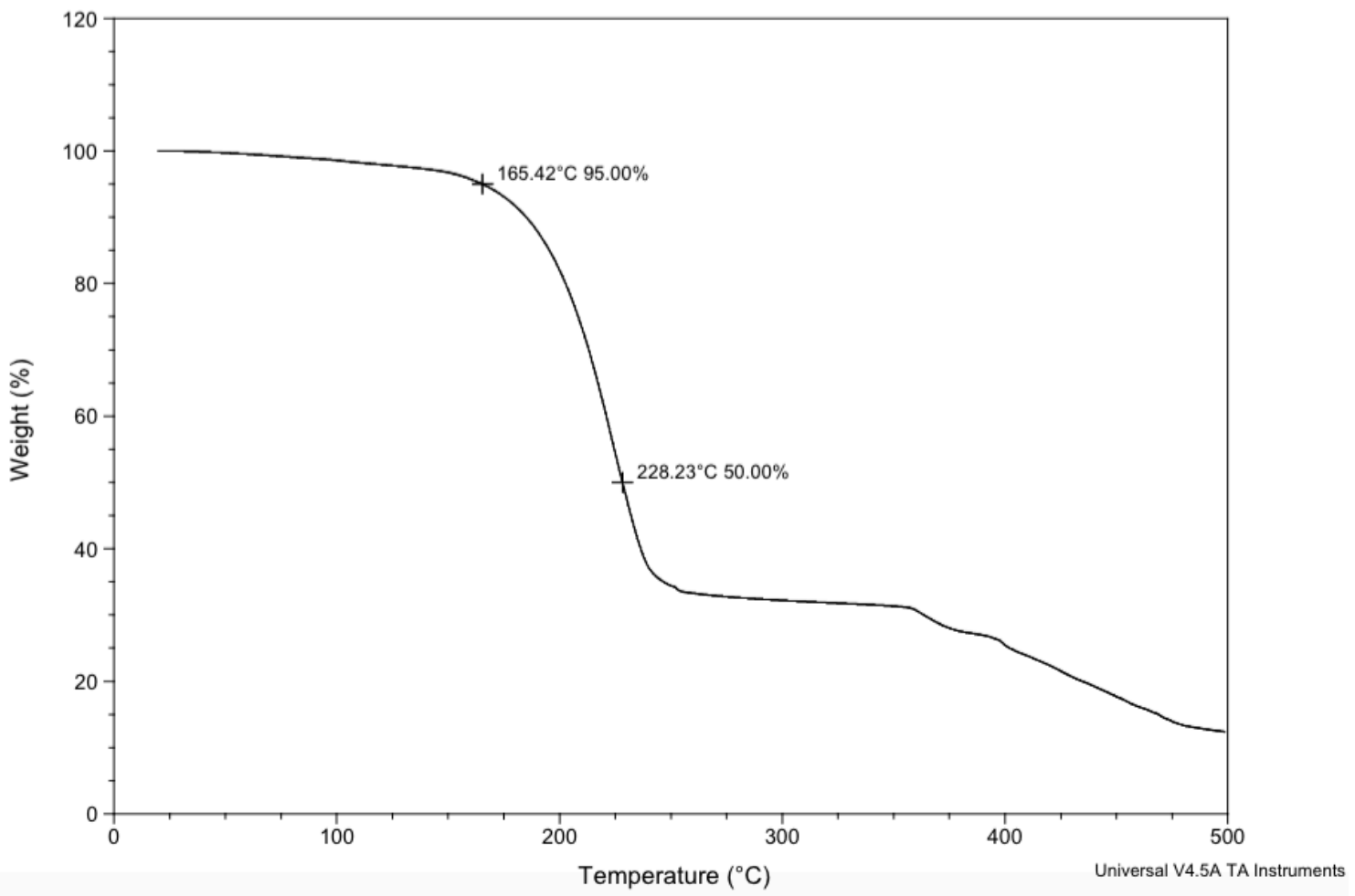

Figure S48. TGA Thermogram of polyethylene hydroquinoate silicon acetal (Table S1, Entry 16). 


\section{${ }^{1}$ H NMR Spectra}

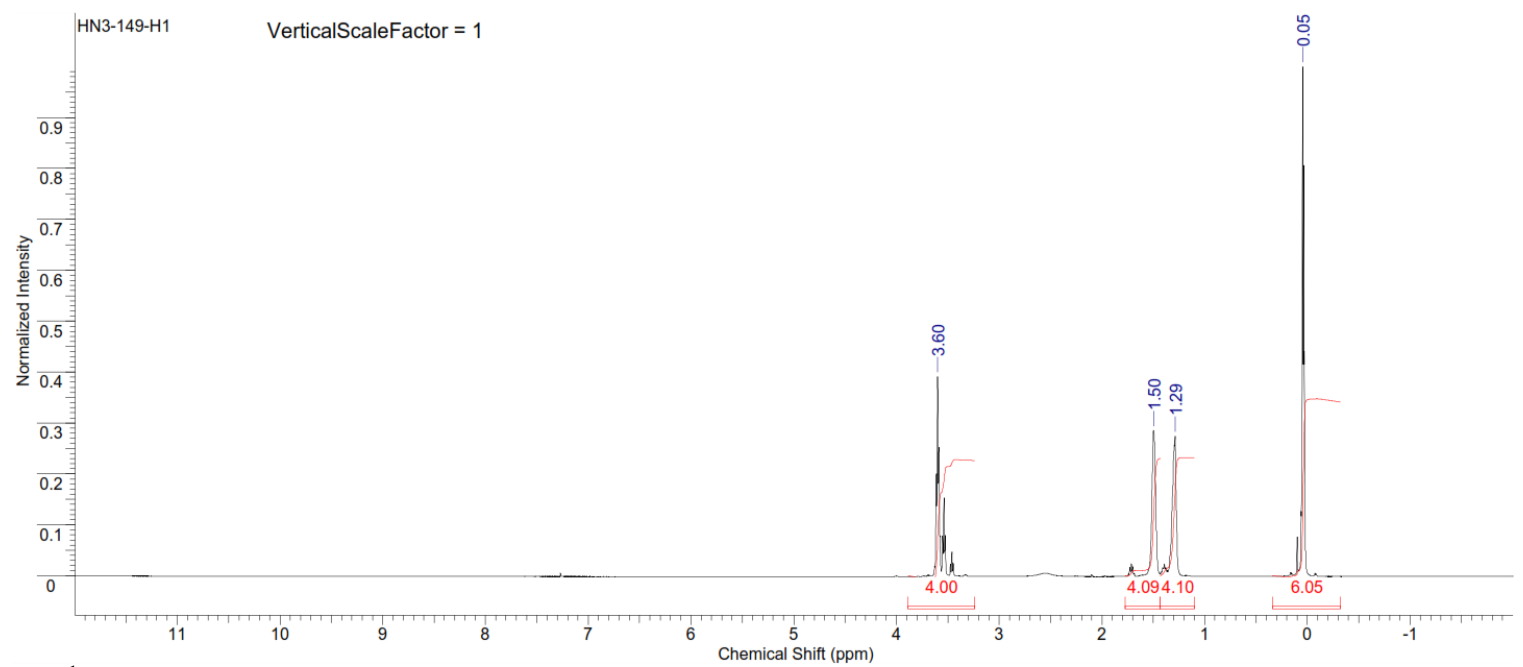

Figure S49. ${ }^{1} \mathrm{H}$ NMR spectrum of polyhexylene silicon acetal (Table S1, entry 1).

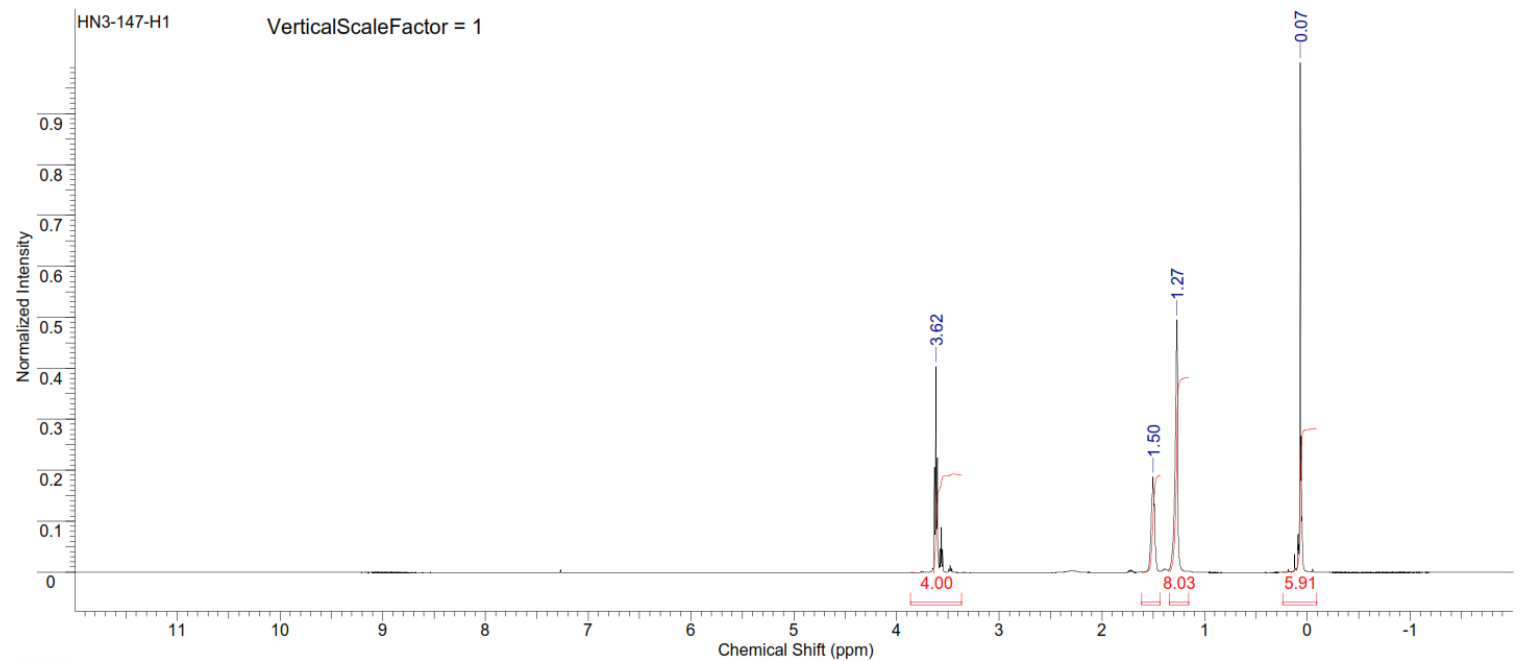

Figure S50. ${ }^{1} \mathrm{H}$ NMR spectrum of polyoctylene silicon acetal (Table S1, entry 2).

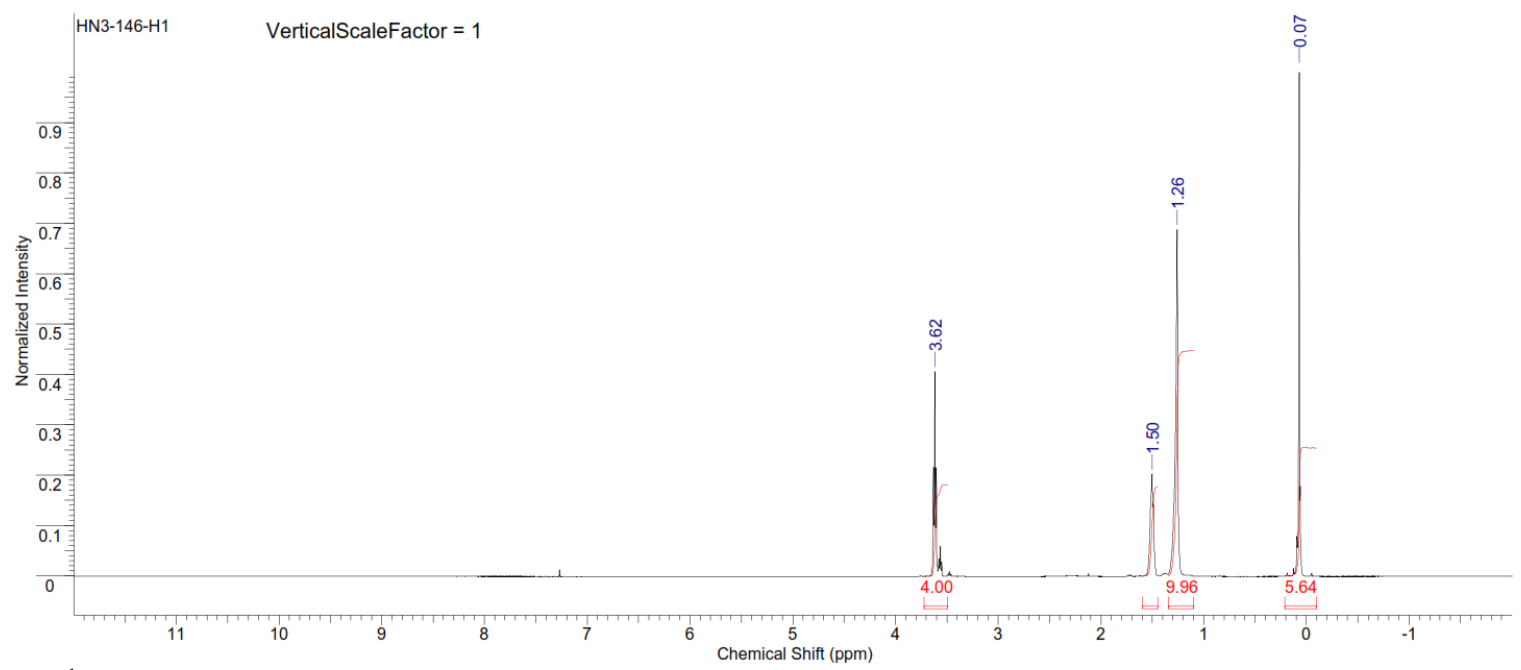

Figure S51. ${ }^{1} \mathrm{H}$ NMR spectrum of polynonylene silicon acetal (Table S1, entry 3). 


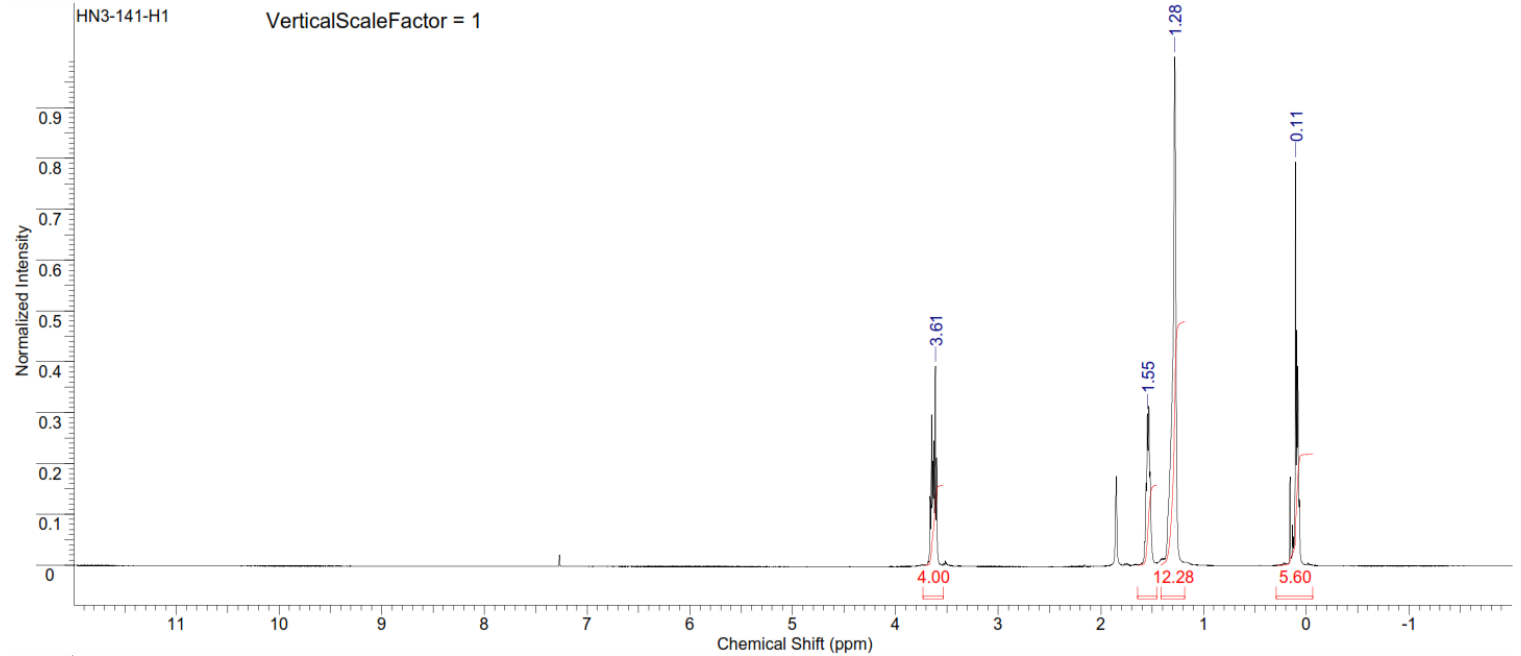

Figure S52. ${ }^{1} \mathrm{H}$ NMR spectrum of polydecylene silicon acetal (Table S1, entry 4).

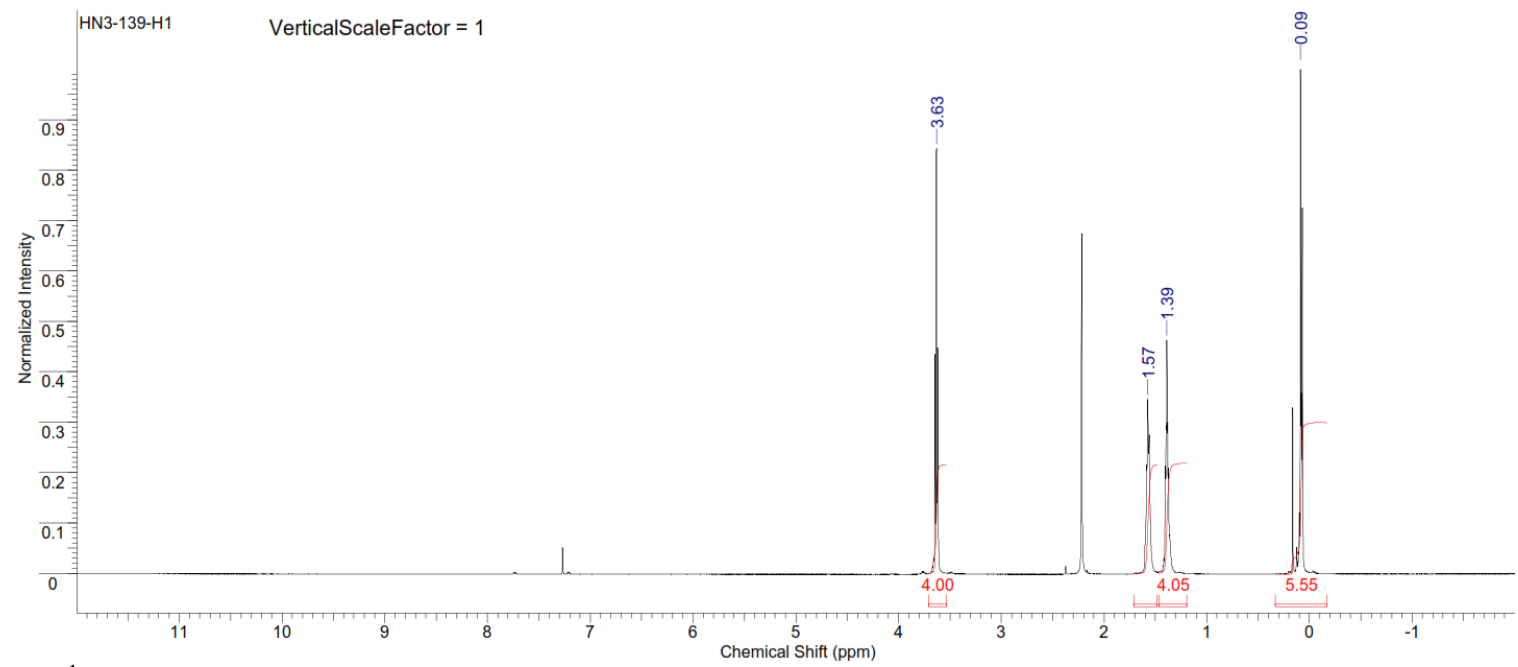

Figure S53. ${ }^{1} \mathrm{H}$ NMR spectrum of polyhexylene silicon acetal (Table S1, entry 5).

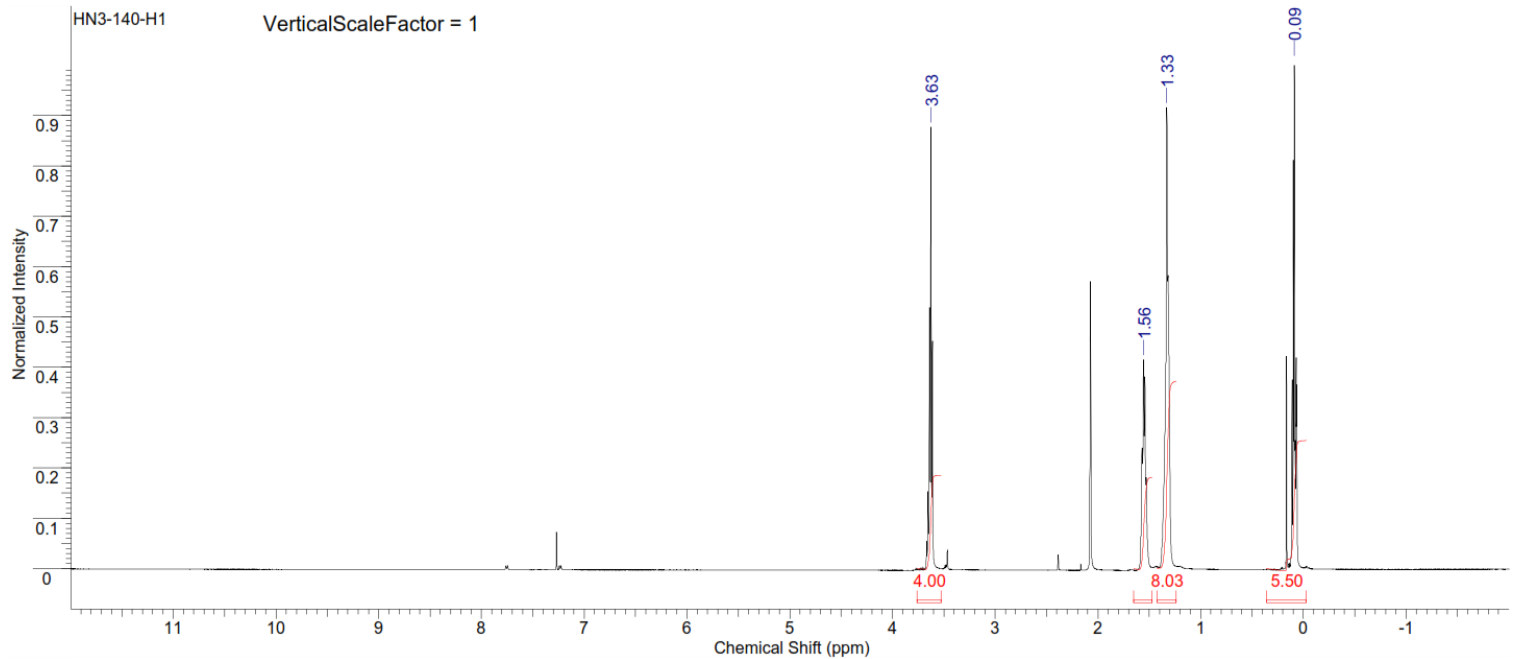

Figure S54. ${ }^{1}$ H NMR spectrum of polyoctylene silicon acetal (Table S1, entry 6). 


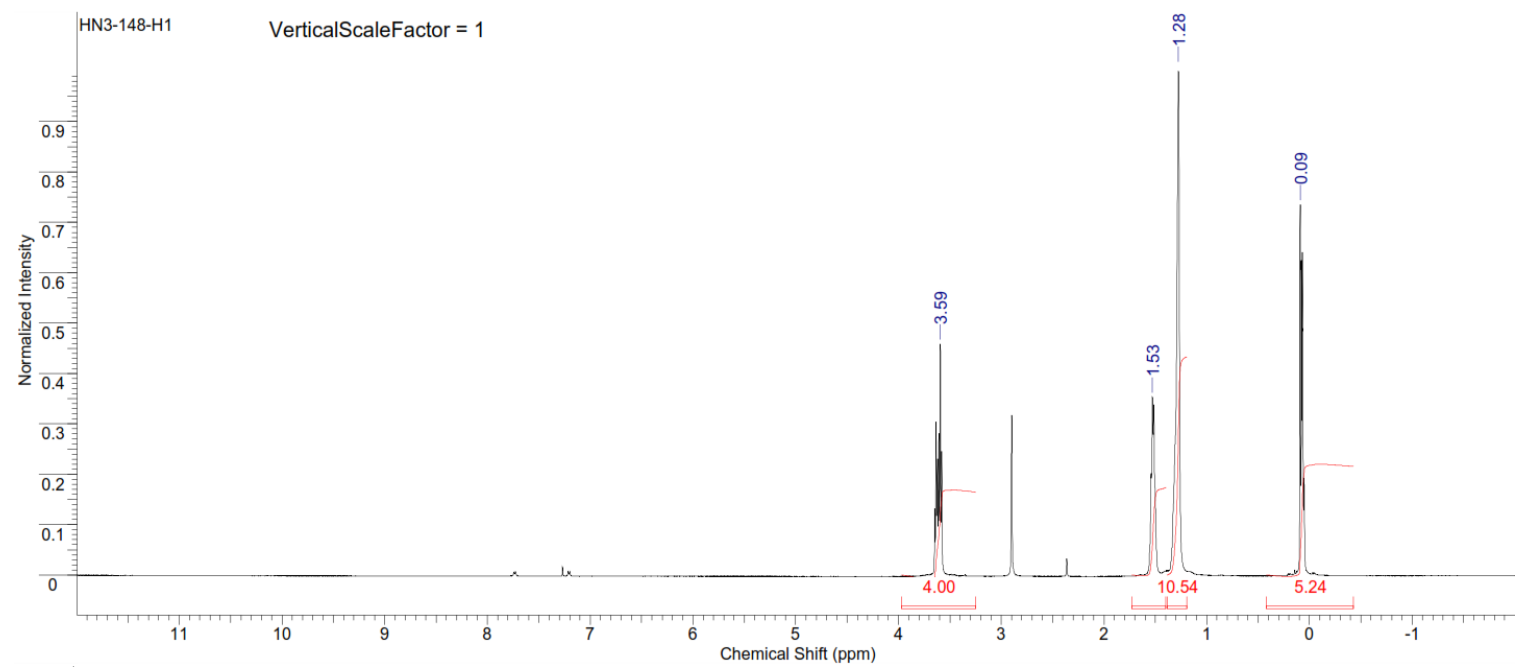

Figure S55. ${ }^{1} \mathrm{H}$ NMR spectrum of polynonylene silicon acetal (Table S1, entry 7).

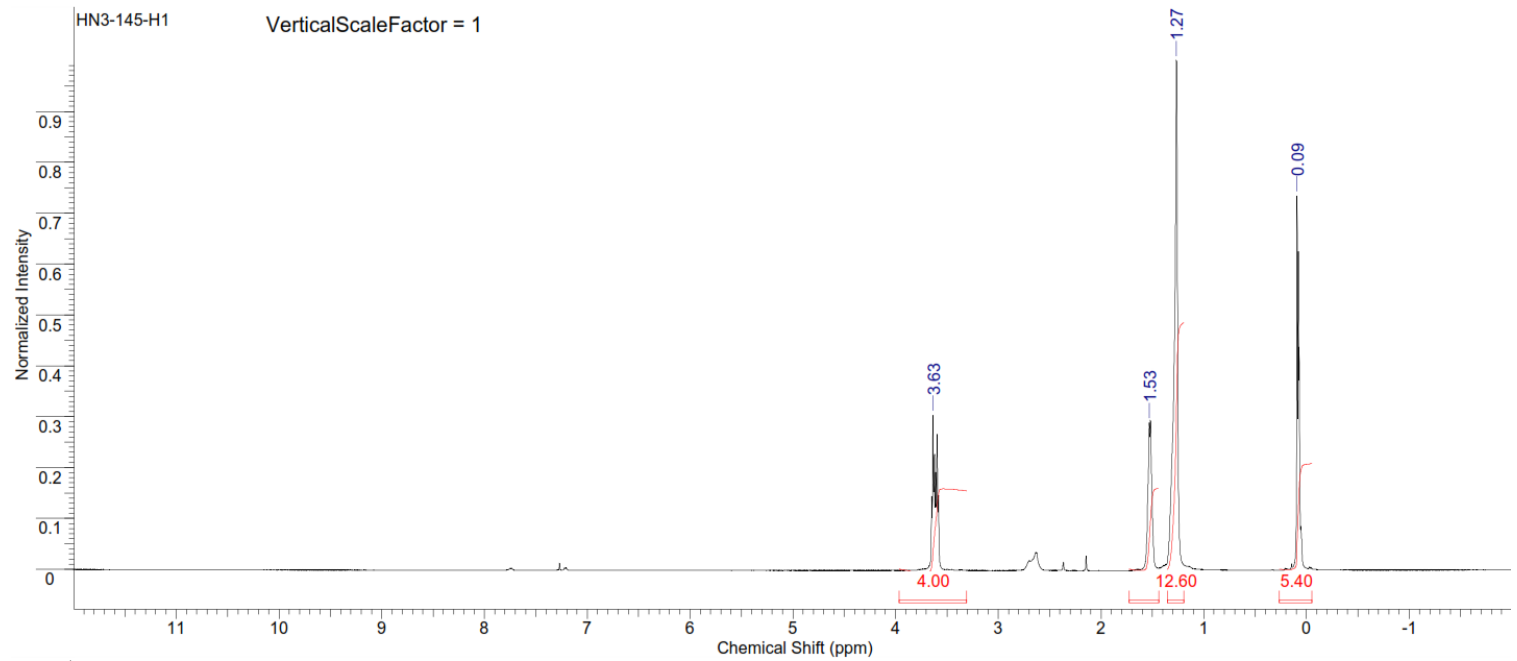

Figure S56. ${ }^{1} \mathrm{H}$ NMR spectrum of polydecylene silicon acetal (Table S1, entry 8).

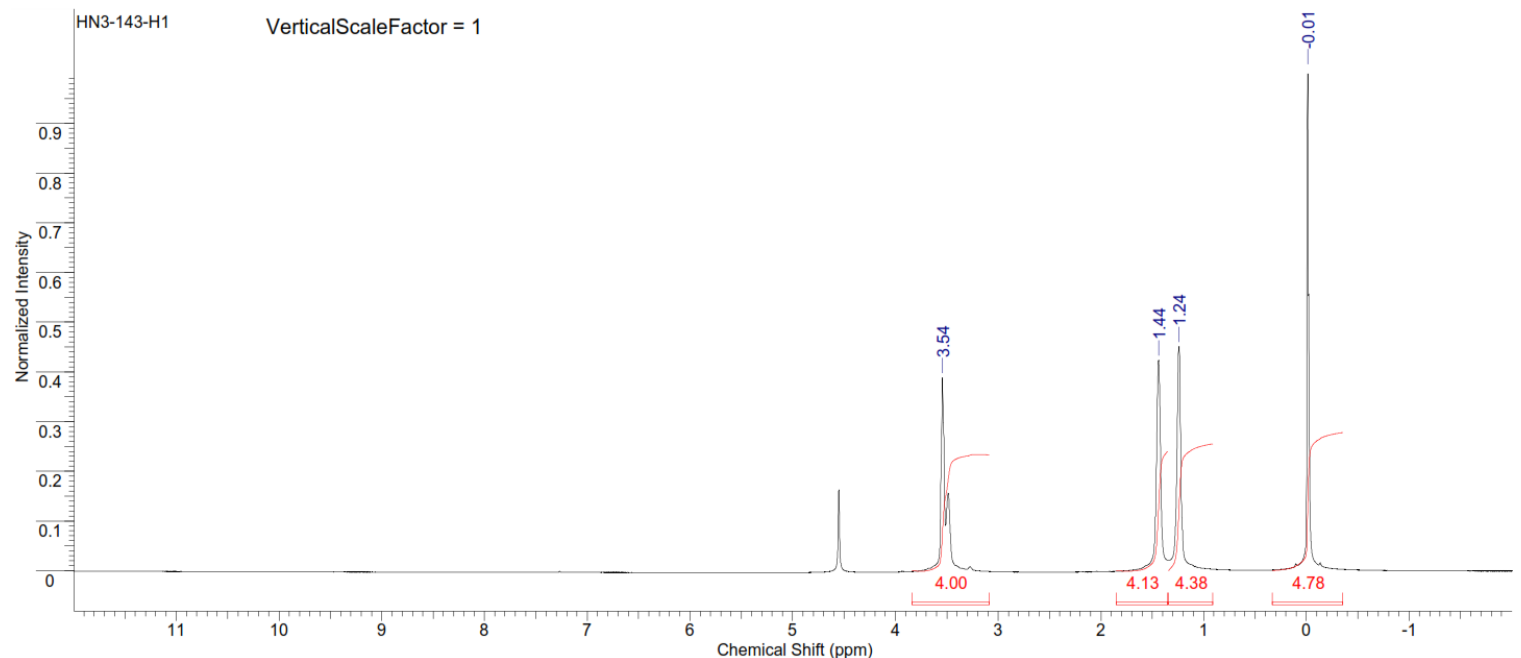

Figure S57. ${ }^{1} \mathrm{H}$ NMR spectrum of polyhexylene silicon acetal (Table S1, entry 9). 


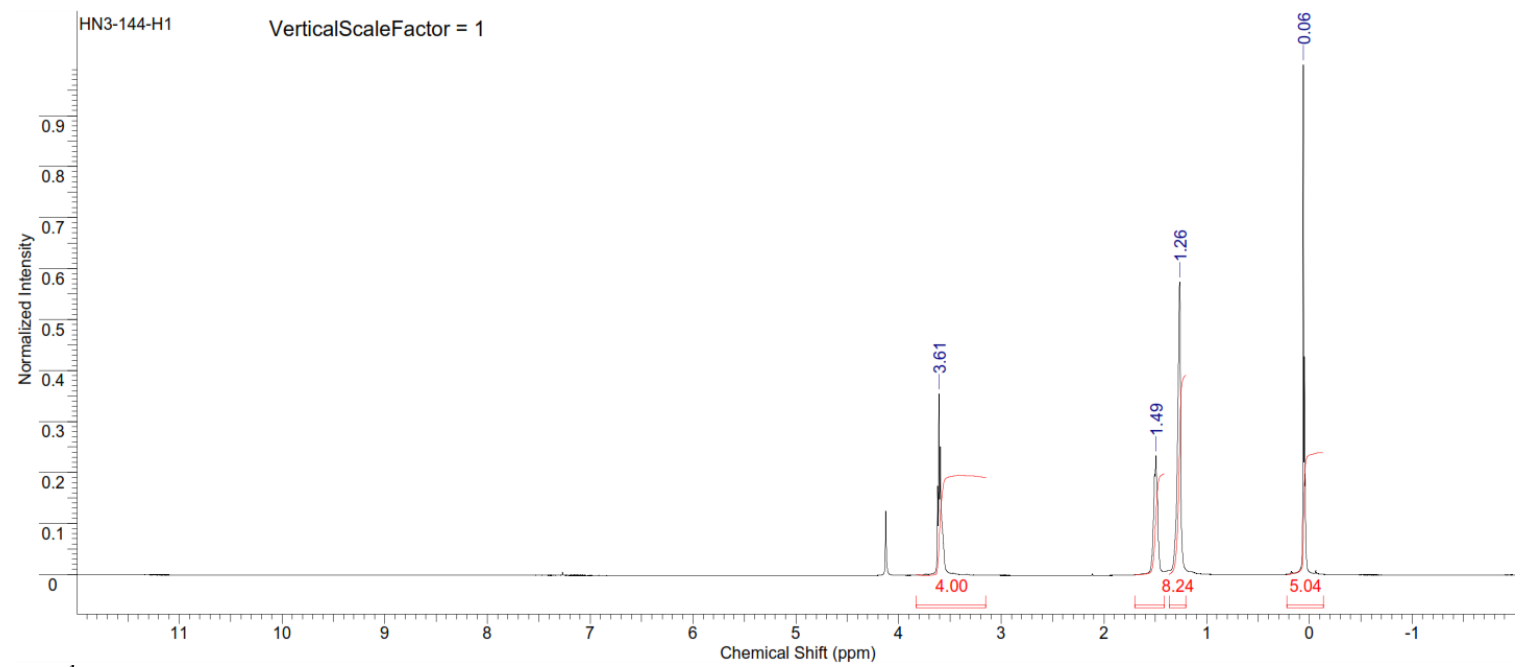

Figure S58. ${ }^{1} \mathrm{H}$ NMR spectrum of polyoctylene silicon acetal (Table S1, entry 10).

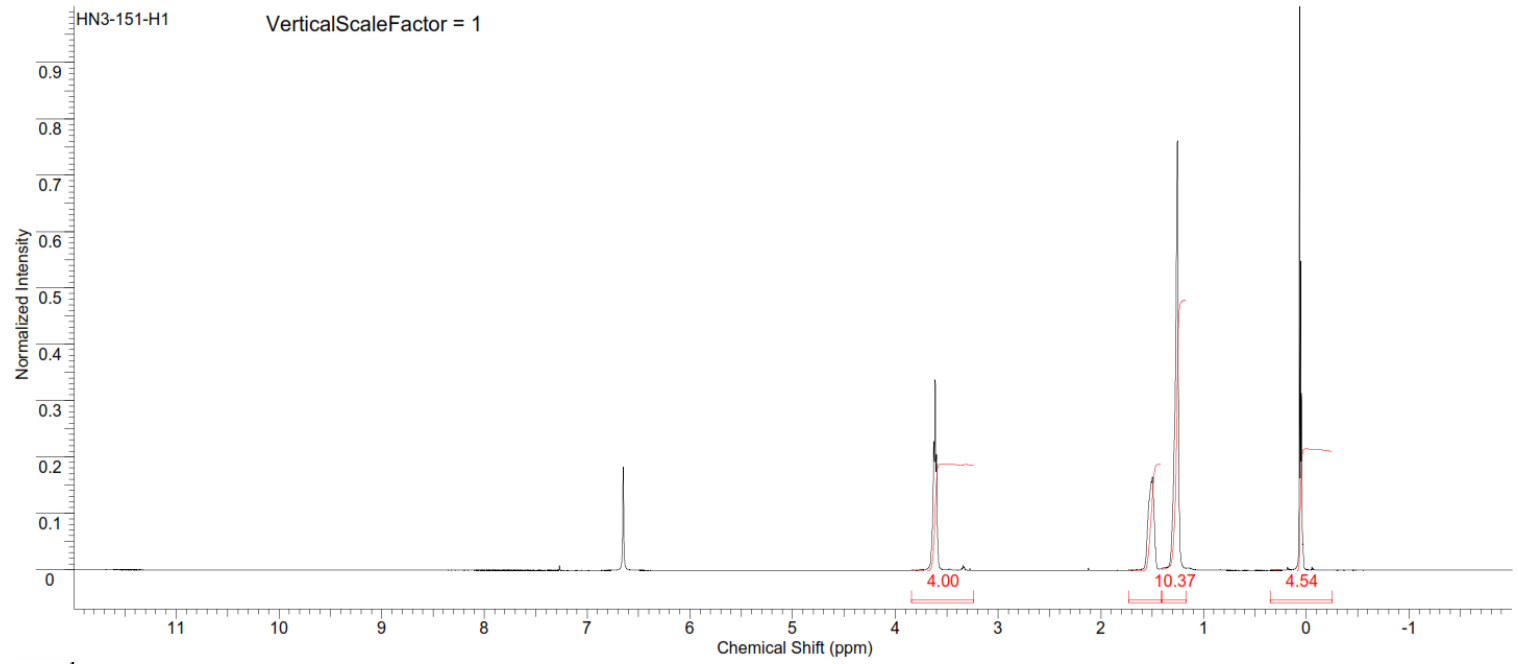

Figure S59. ${ }^{1} \mathrm{H}$ NMR spectrum of polynonylene silicon acetal (Table S1, entry 11).

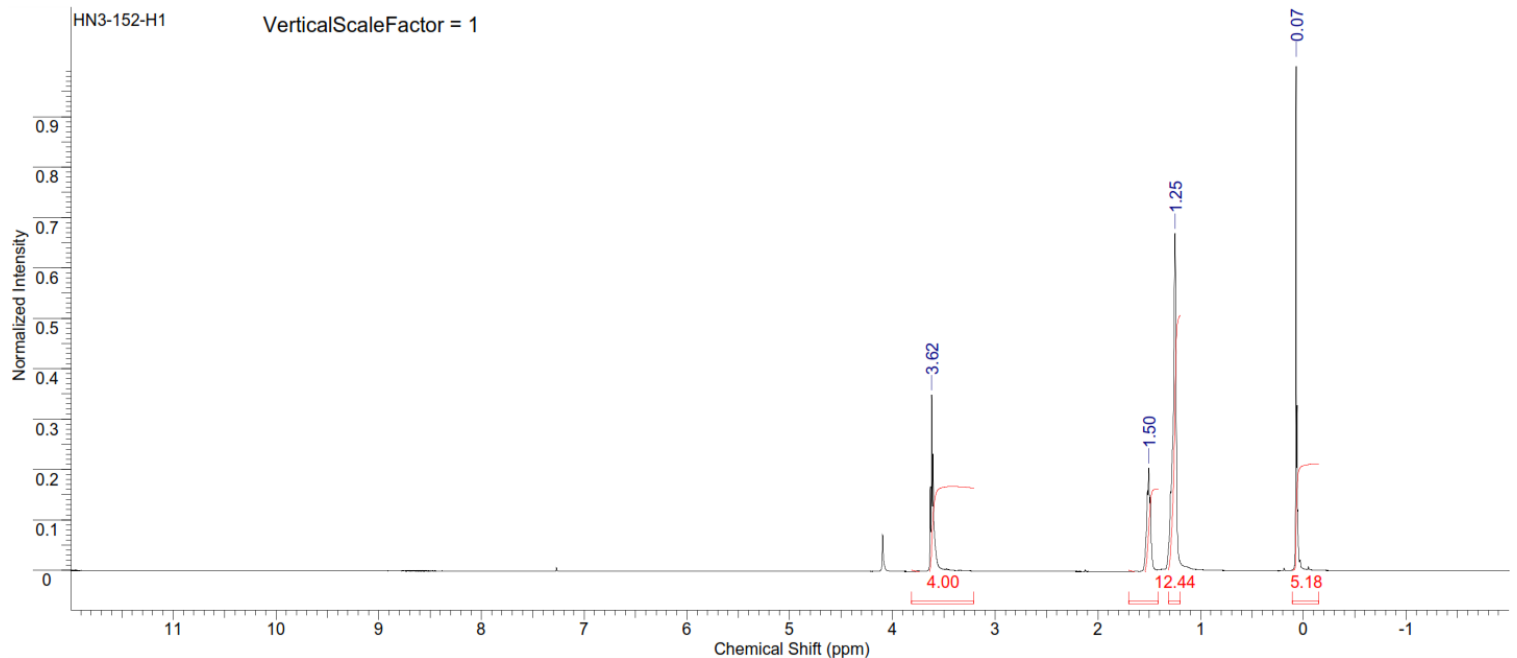

Figure S60. ${ }^{1} \mathrm{H}$ NMR spectrum of polydecylene silicon acetal (Table S1, entry 12). 


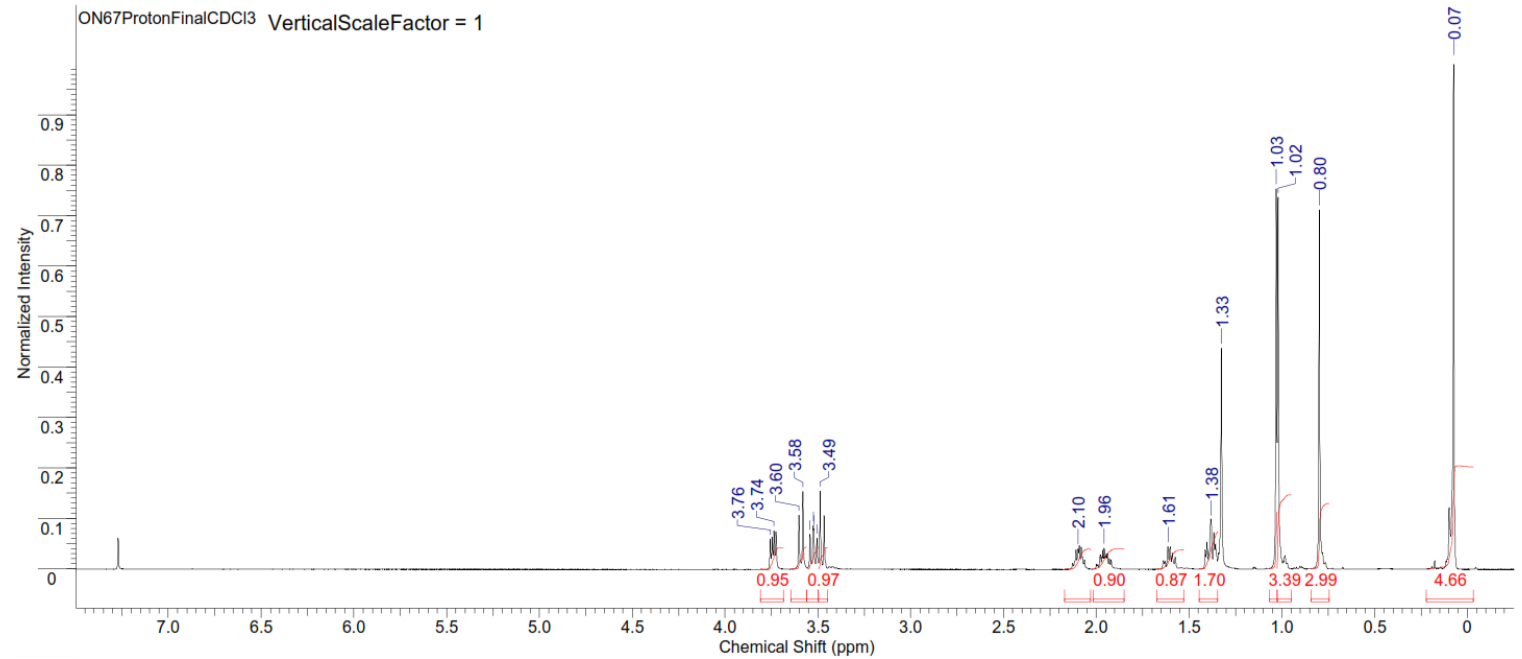

Figure S61. ${ }^{1}$ H NMR spectrum of polycamphoric silicon acetal (Table S1, entry 13).

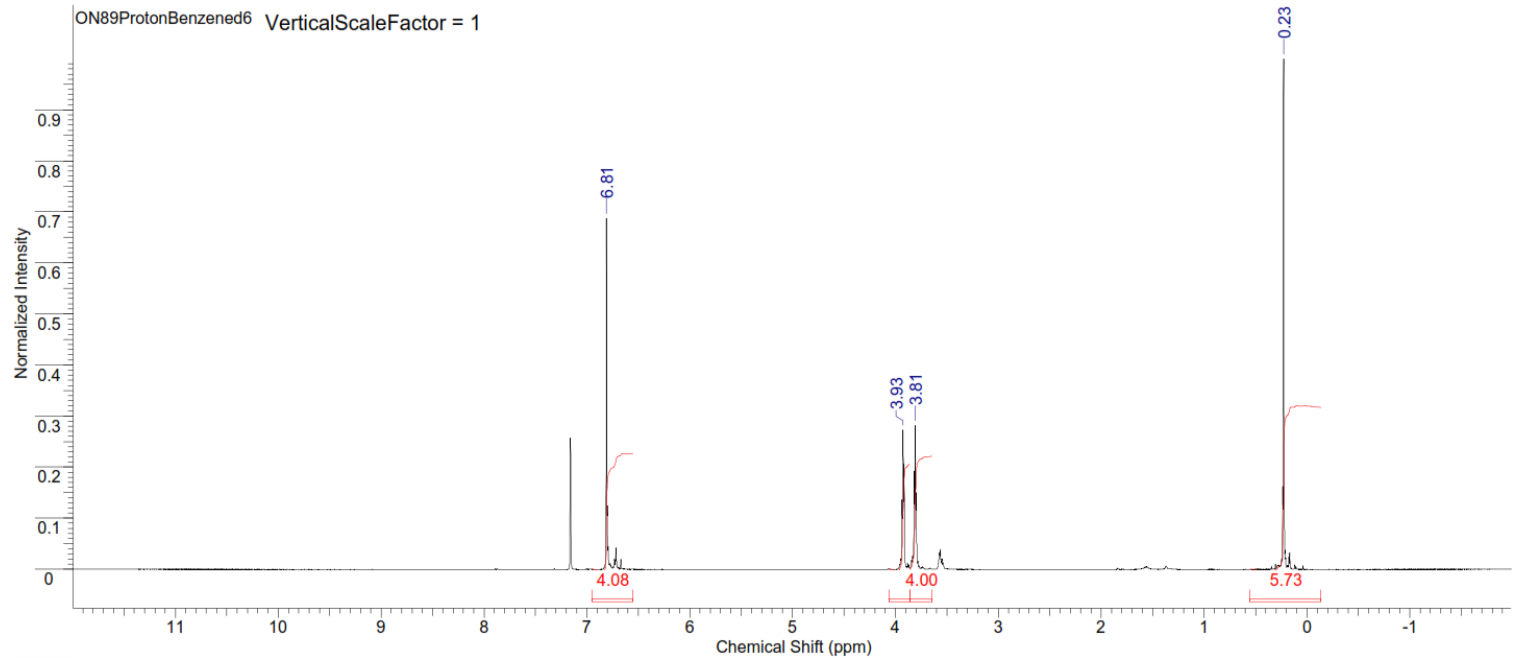

Figure S62. ${ }^{1}$ H NMR spectrum of polyethylene hydroquinonoate silicon acetal (Table S1, entry 14).

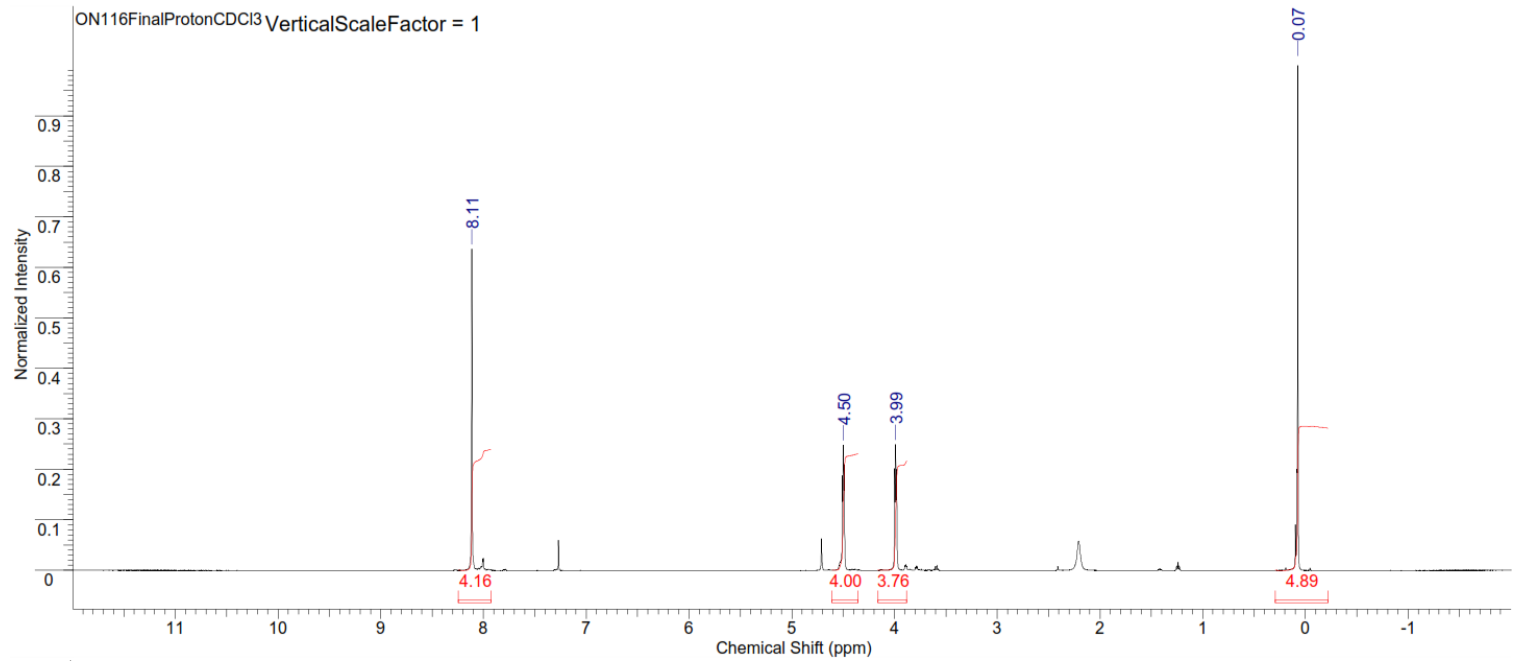

Figure S63. ${ }^{1} \mathrm{H}$ NMR spectrum of polyethylene terephthalate silicon acetal (Table S1, entry 15). 


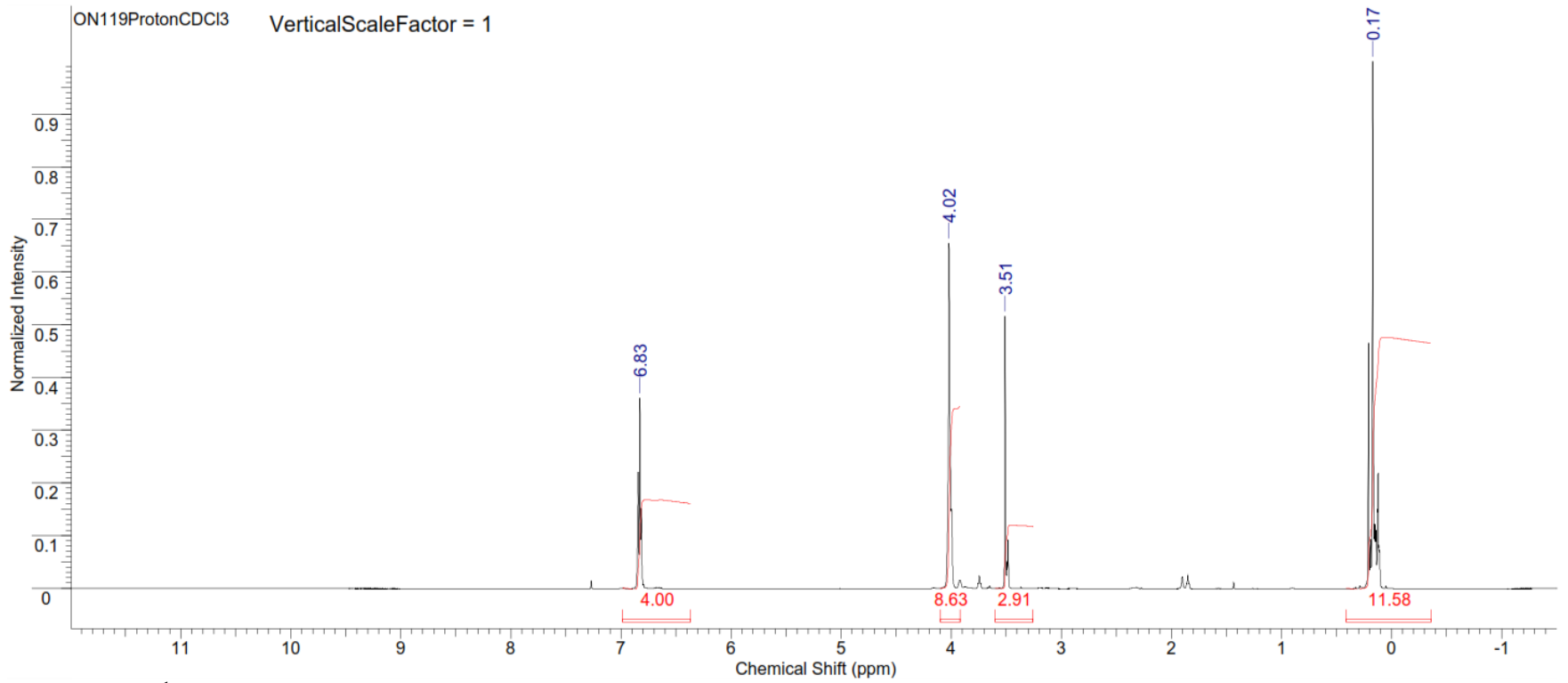

Figure S64. ${ }^{1}$ H NMR spectrum of 1,4-bis(2-((methoxydimethylsilyl)oxy)ethoxy)benzene (bis-silicon acetal monomer).

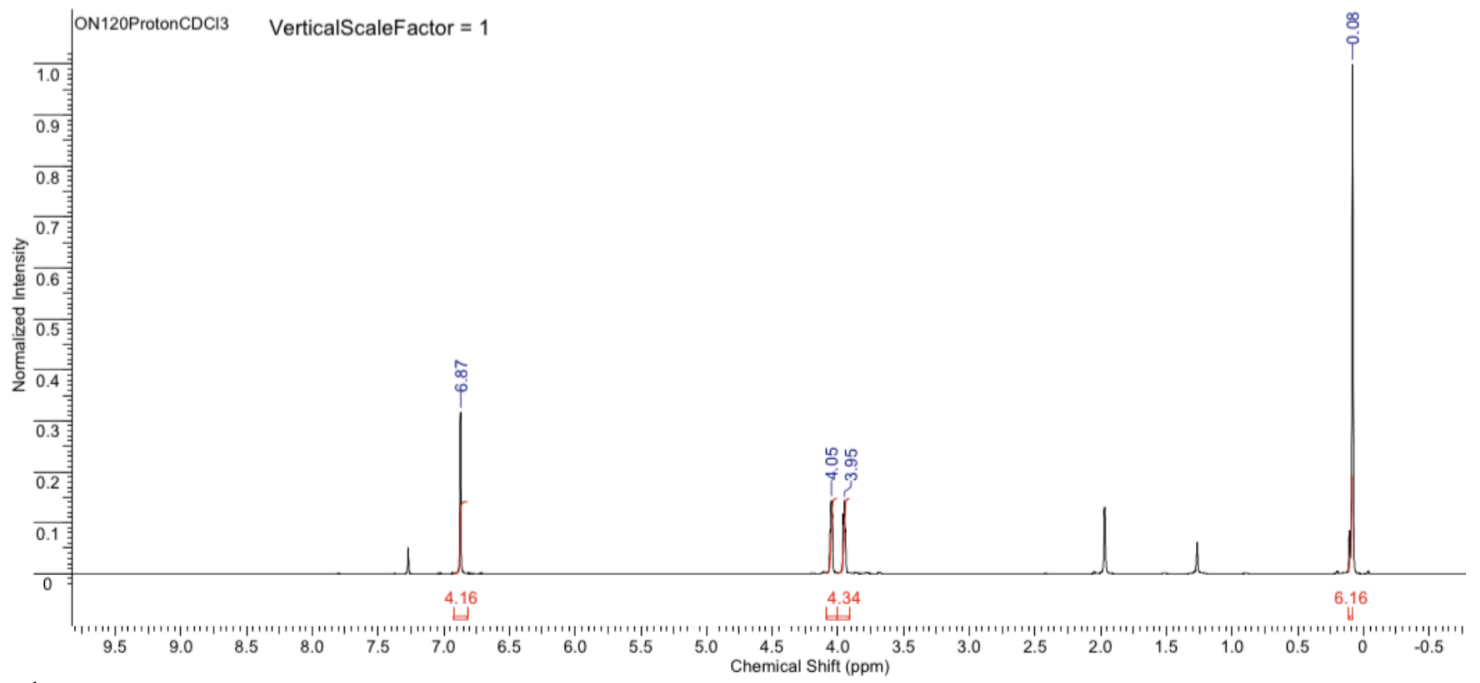

Figure S65. ${ }^{1}$ H NMR spectrum of polyethylene hydroquinonoate silicon acetal (Table S1, entry 16). 


\section{${ }^{13}$ C NMR Spectra}

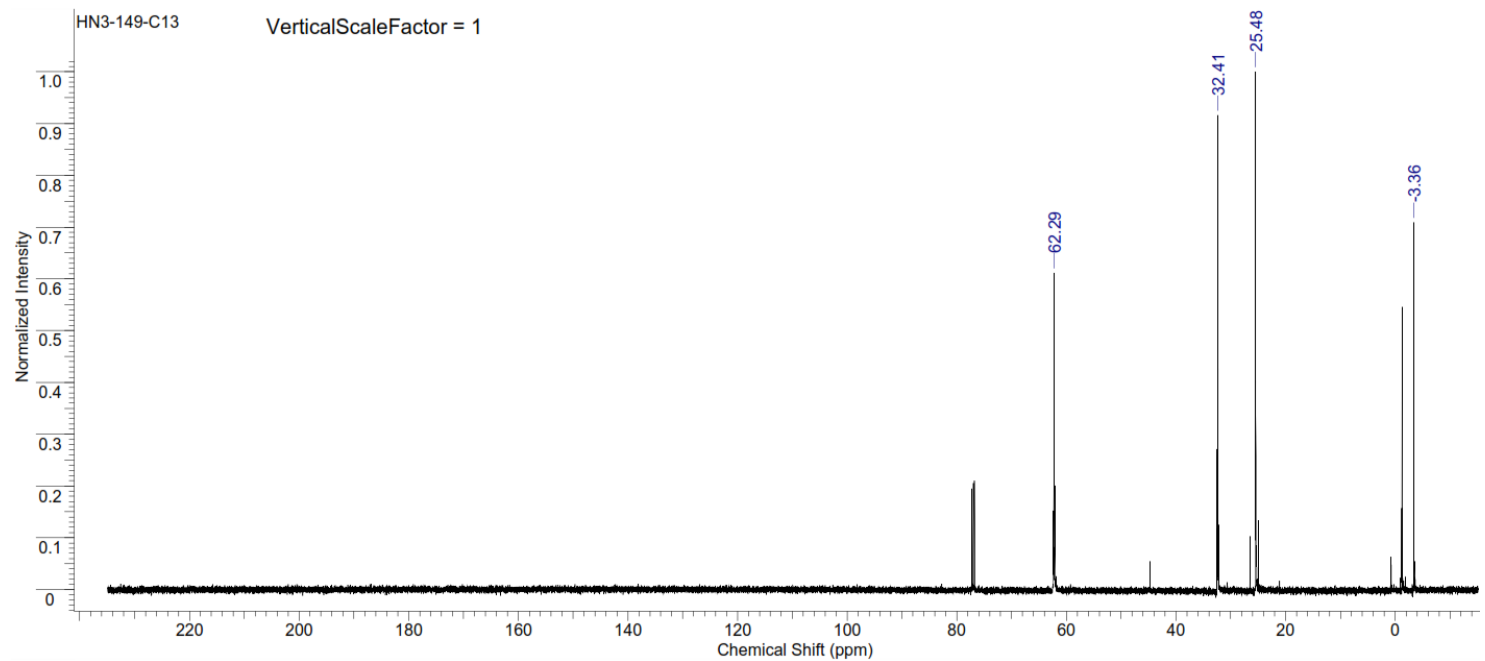

Figure S66. ${ }^{13} \mathrm{C}$ NMR spectrum of polyhexylene silicon acetal (Table S1, entry 1).

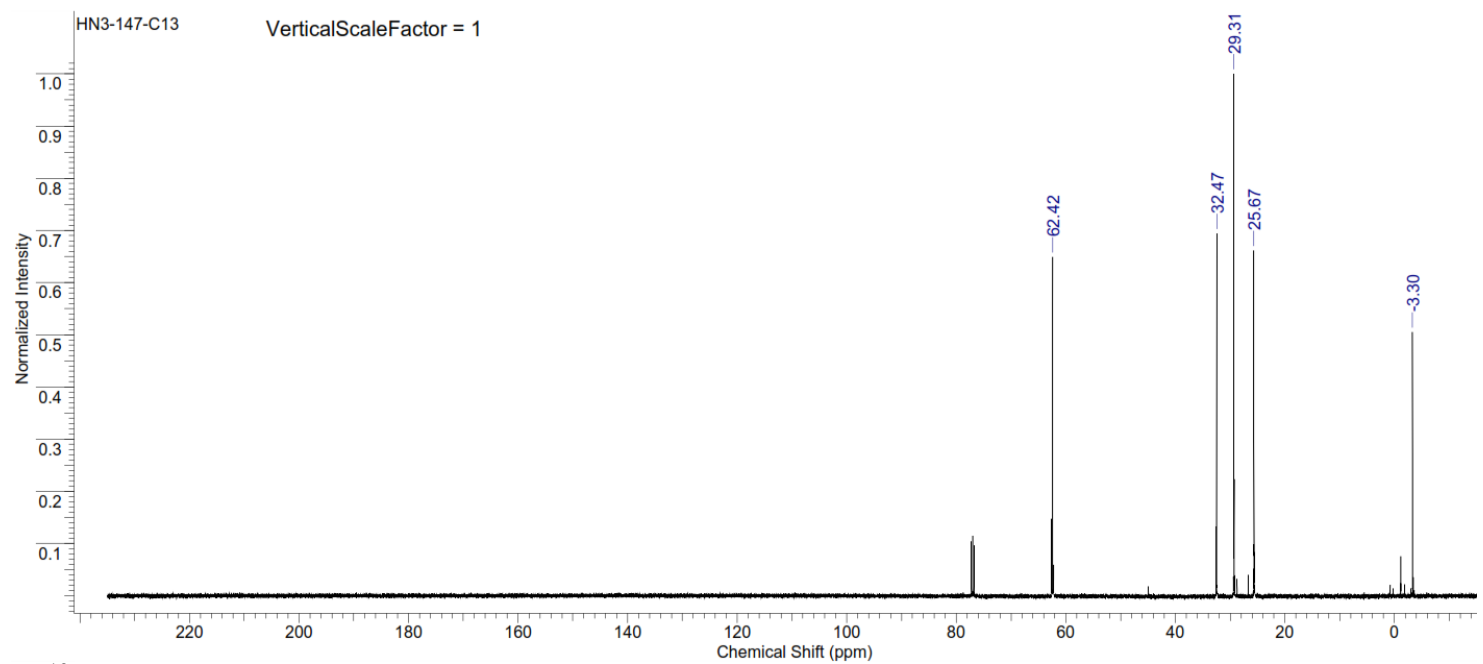

Figure S67. ${ }^{13} \mathrm{C}$ NMR spectrum of polyoctylene silicon acetal (Table S1, entry 2).

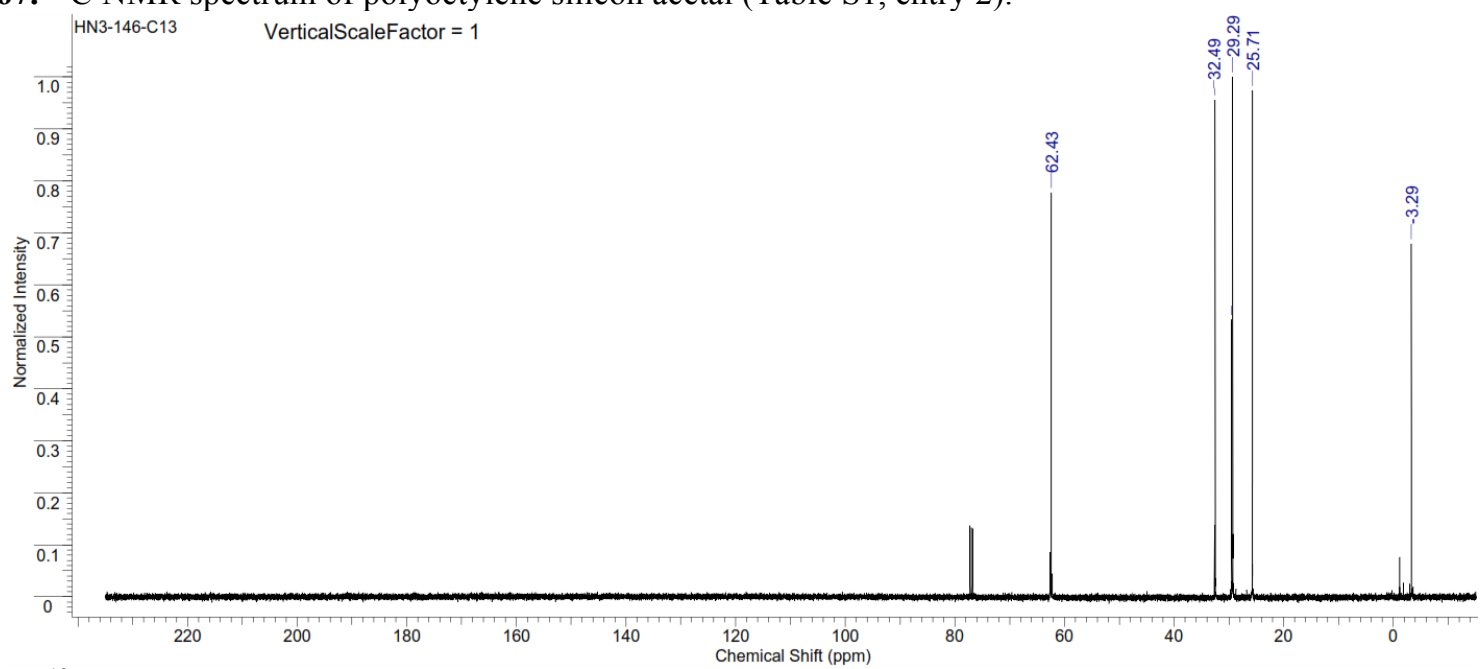

Figure S68. ${ }^{13} \mathrm{C}$ NMR spectrum of polynonylene silicon acetal (Table S1, entry 3). 


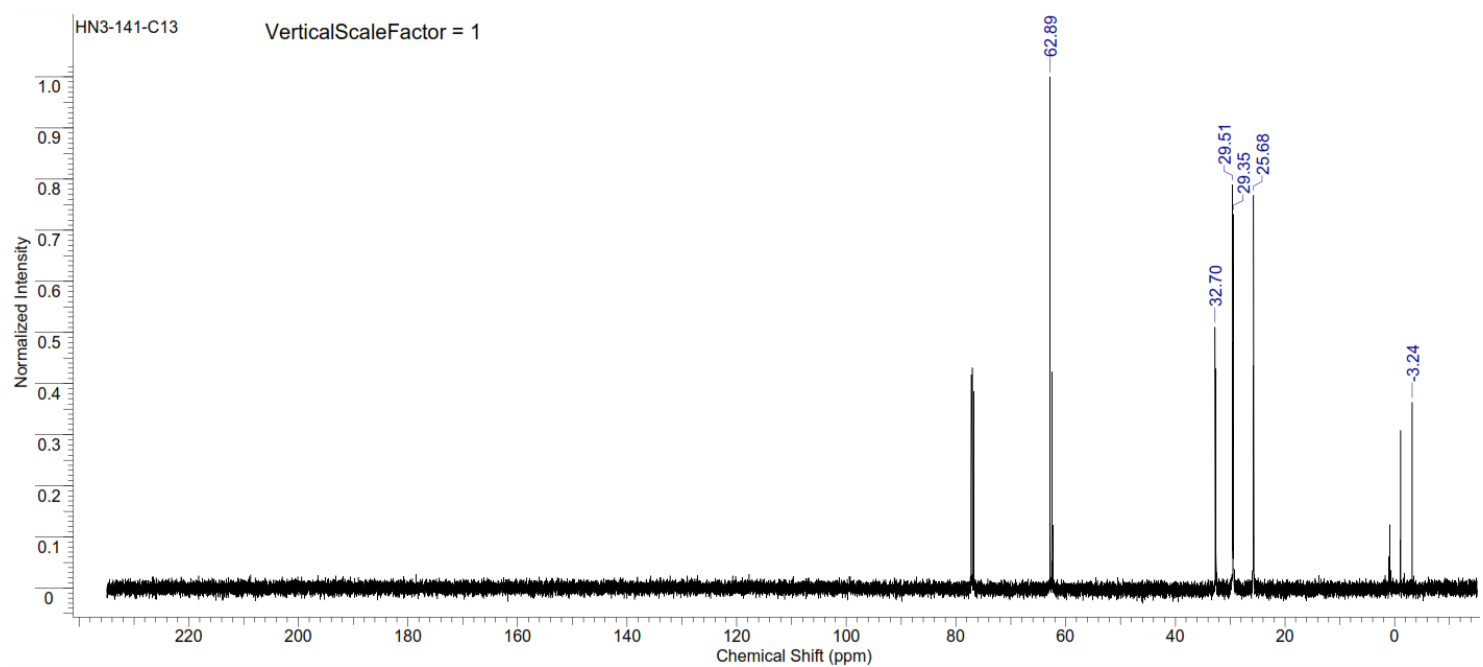

Figure S69. ${ }^{13} \mathrm{C}$ NMR spectrum of polydecylene silicon acetal (Table S1, entry 4).

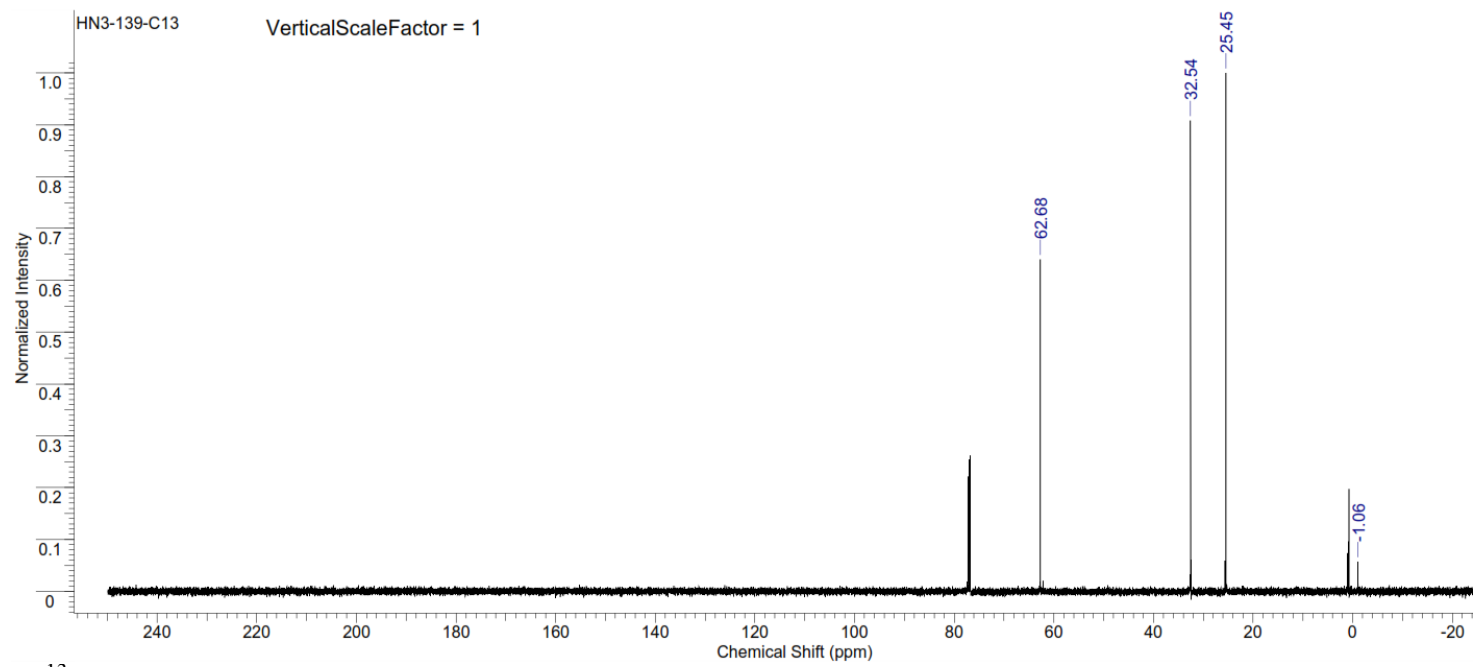

Figure S70. ${ }^{13} \mathrm{C}$ NMR spectrum of polyhexylene silicon acetal (Table S1, entry 5).

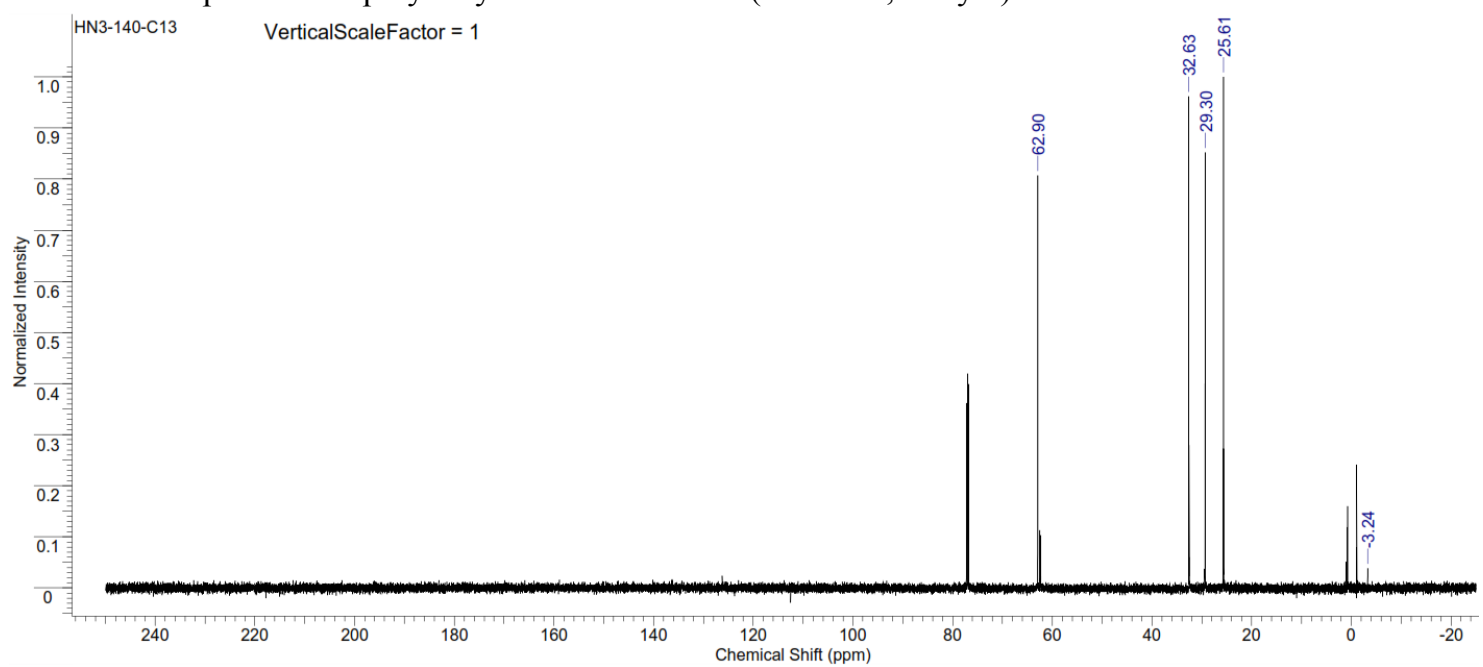

Figure S71. ${ }^{13} \mathrm{C}$ NMR spectrum of polyoctylene silicon acetal (Table S1, entry 6). 


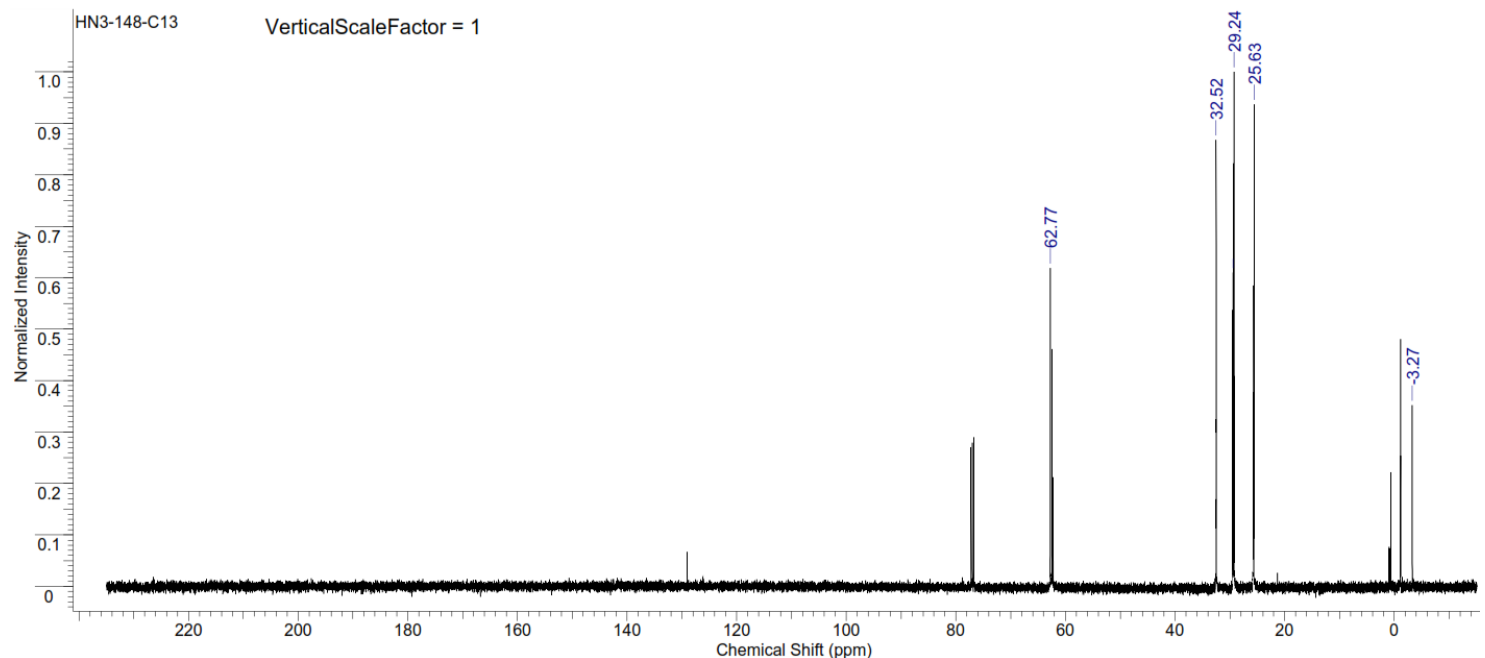

Figure S72. ${ }^{13} \mathrm{C}$ NMR spectrum of polynonylene silicon acetal (Table S1, entry 7).

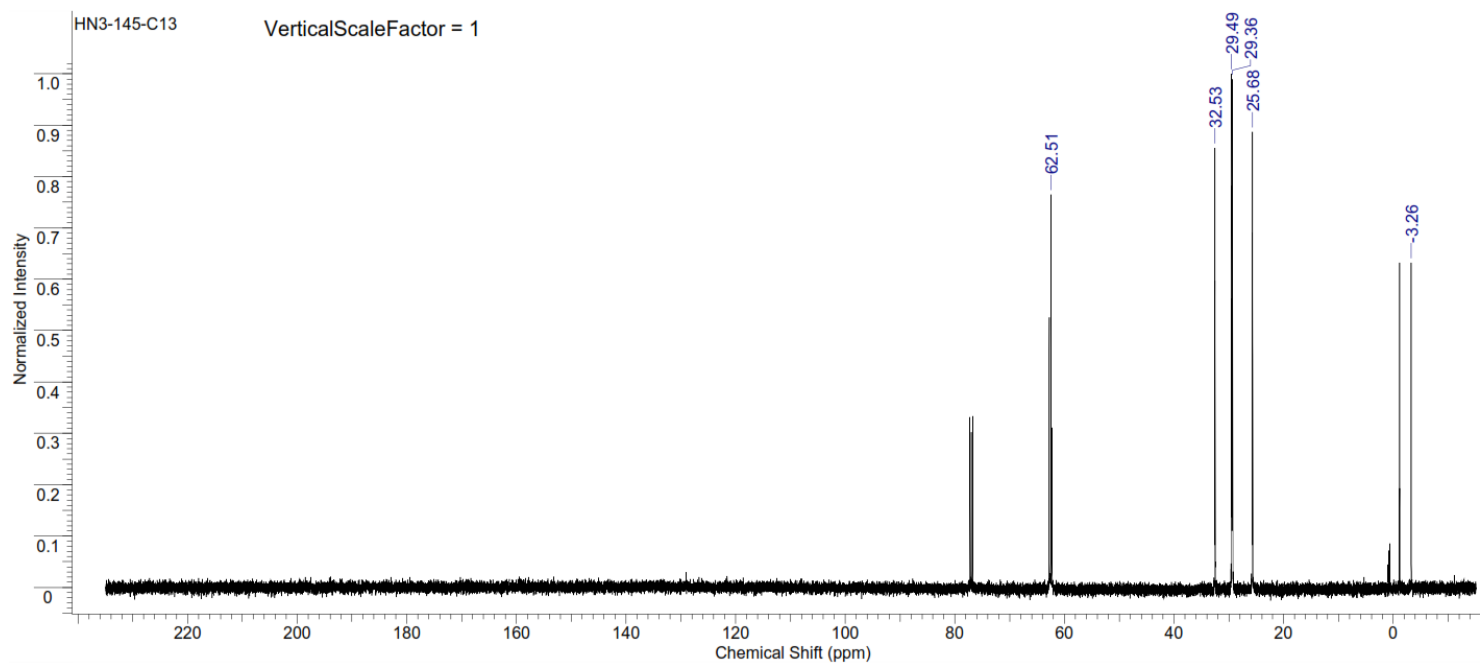

Figure S73. ${ }^{13} \mathrm{C}$ NMR spectrum of polydecylene silicon acetal (Table S1, entry 8).

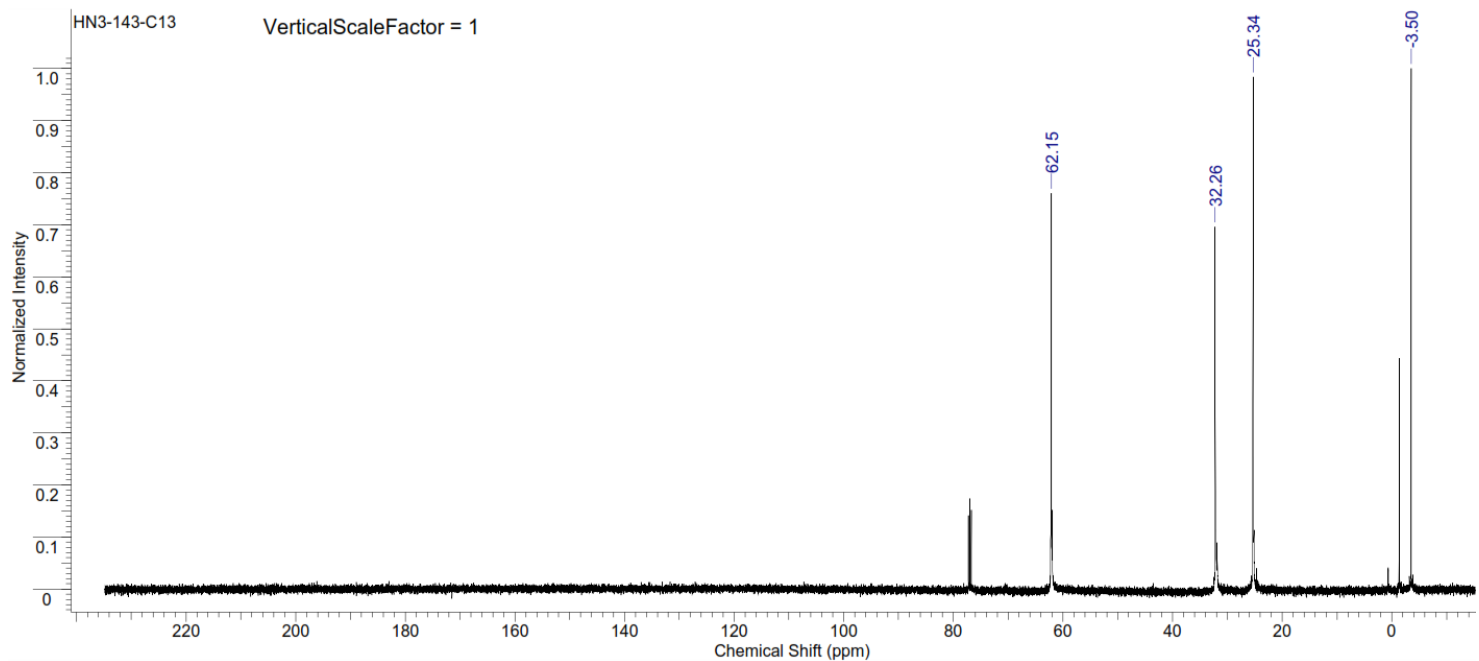

Figure S74. ${ }^{13} \mathrm{C}$ NMR spectrum of polyhexylene silicon acetal (Table S1, entry 9). 


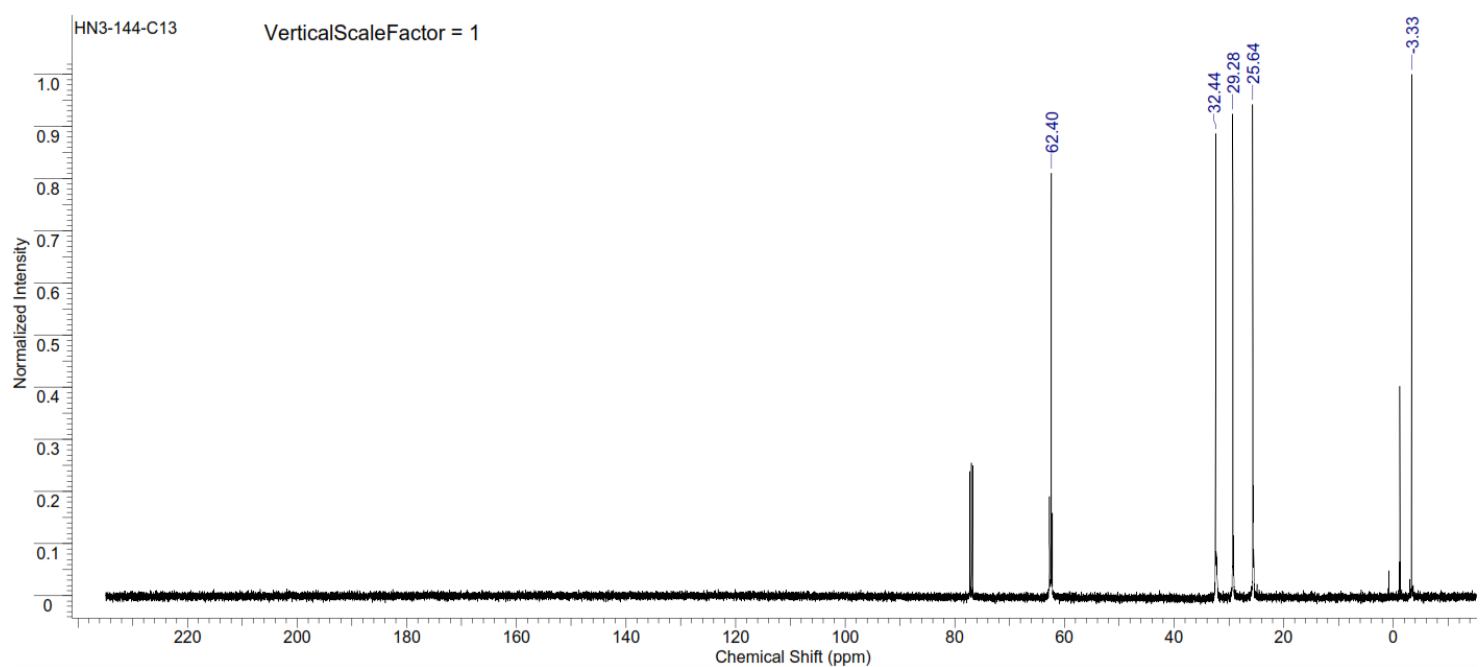

Figure S75. ${ }^{13} \mathrm{C}$ NMR spectrum of polyoctylene silicon acetal (Table S1, entry 10).

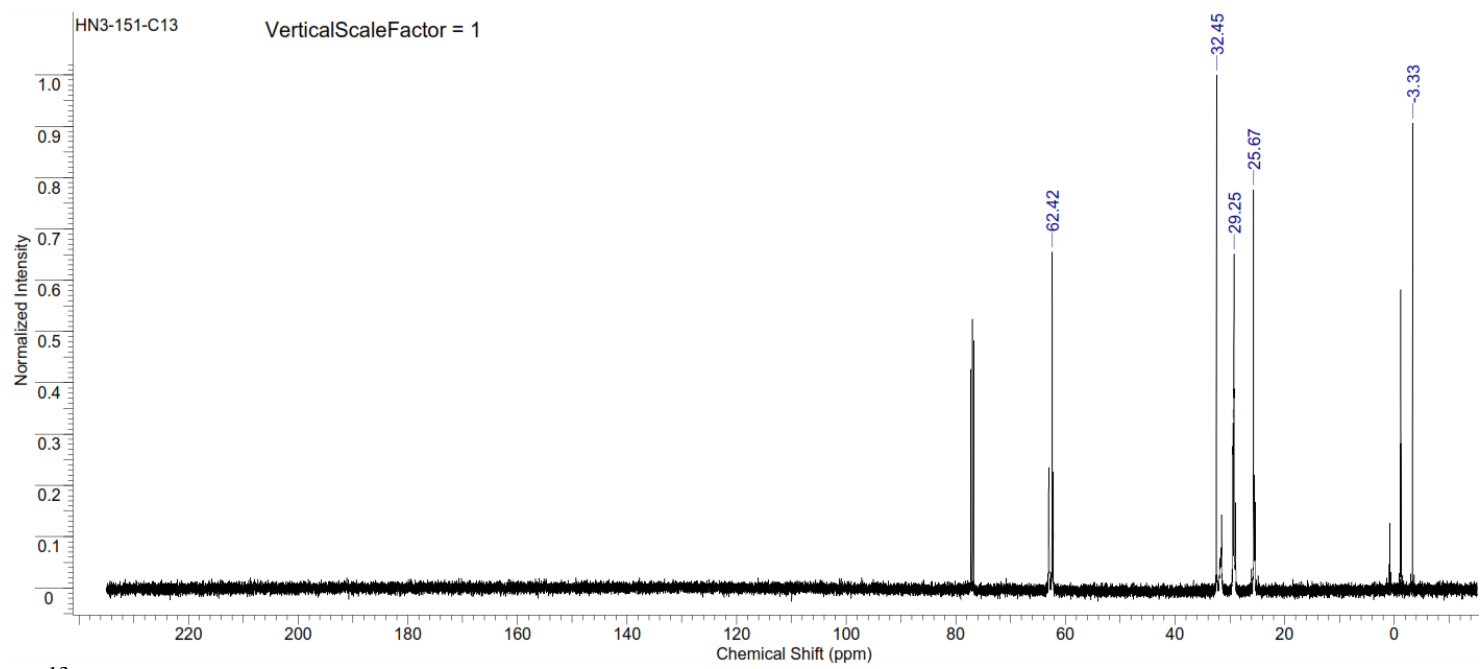

Figure S76. ${ }^{13} \mathrm{C}$ NMR spectrum of polynonylene silicon acetal (Table S1, entry 11).

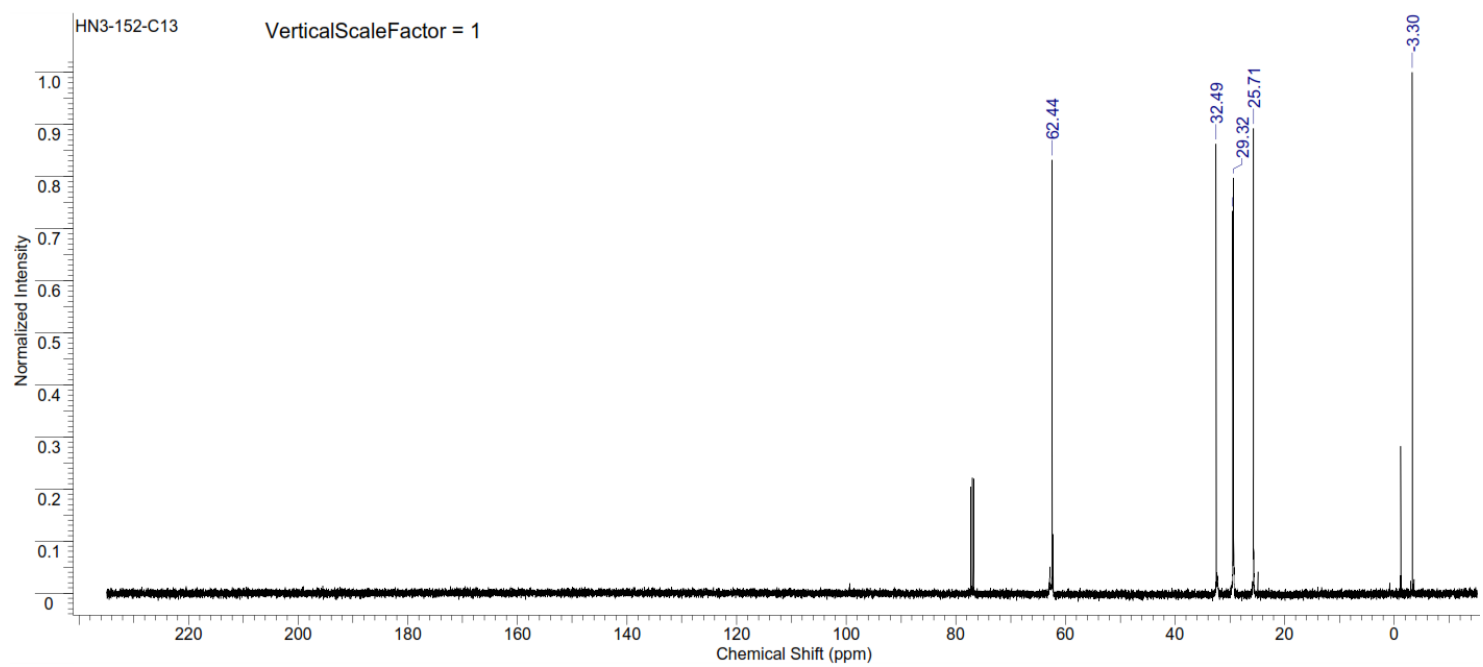

Figure S77. ${ }^{13} \mathrm{C}$ NMR spectrum of polydecylene silicon acetal (Table S1, entry 12). 


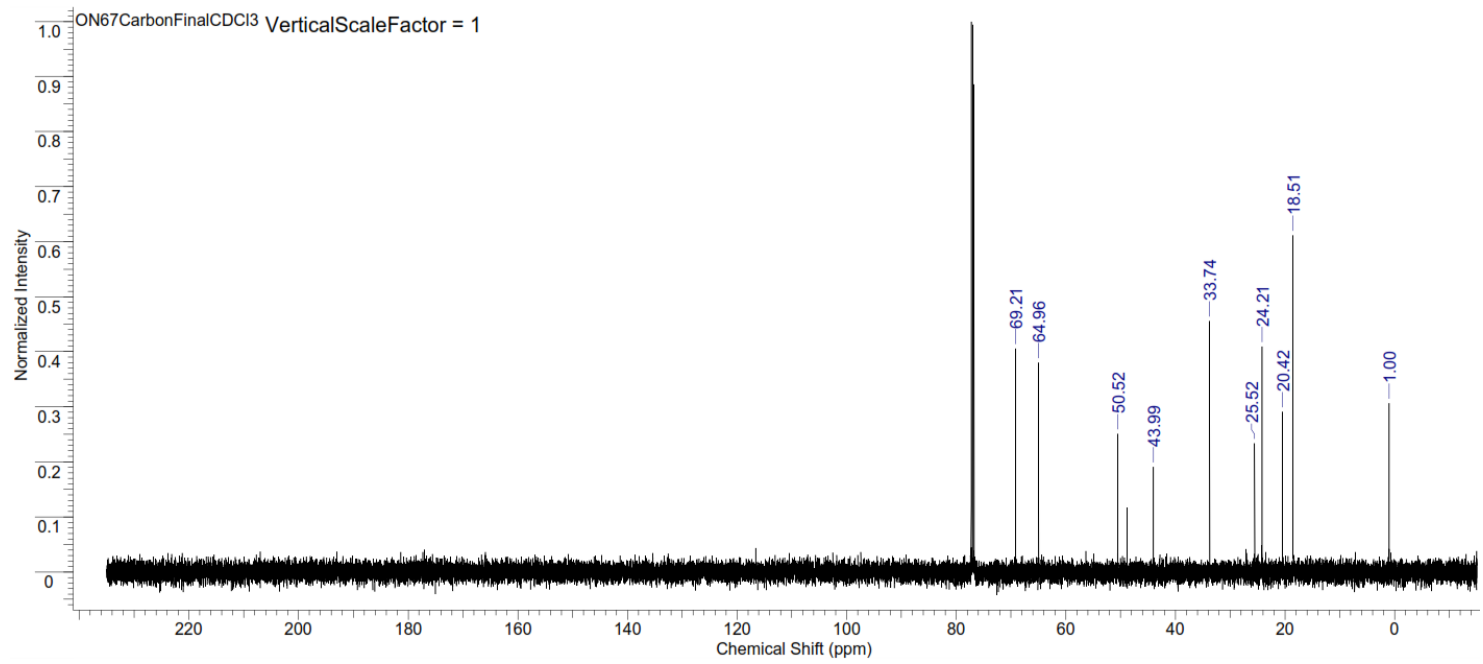

Figure S78. ${ }^{13} \mathrm{C}$ NMR spectrum of polycamphoric silicon acetal (Table S1, entry 13).

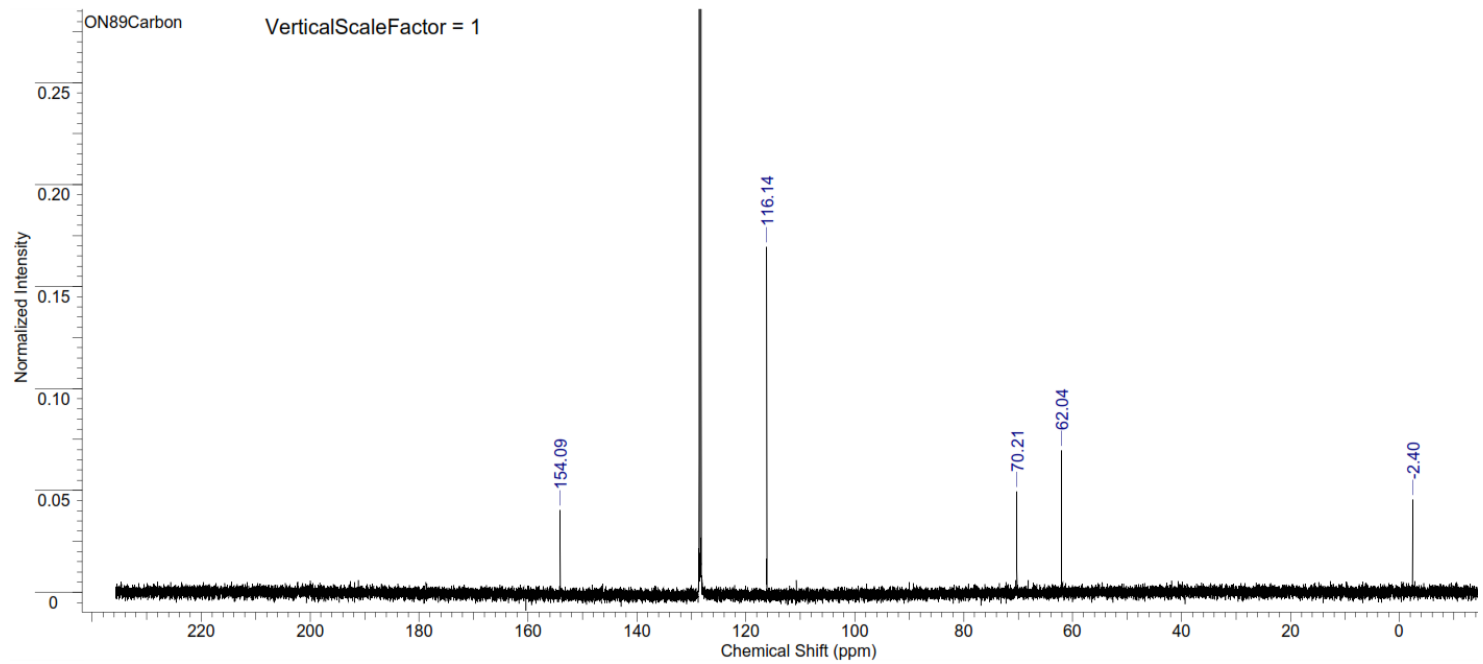

Figure S79. ${ }^{13} \mathrm{C}$ NMR spectrum of polyethylene hydroquinonoate silicon acetal (Table S1, entry 14).

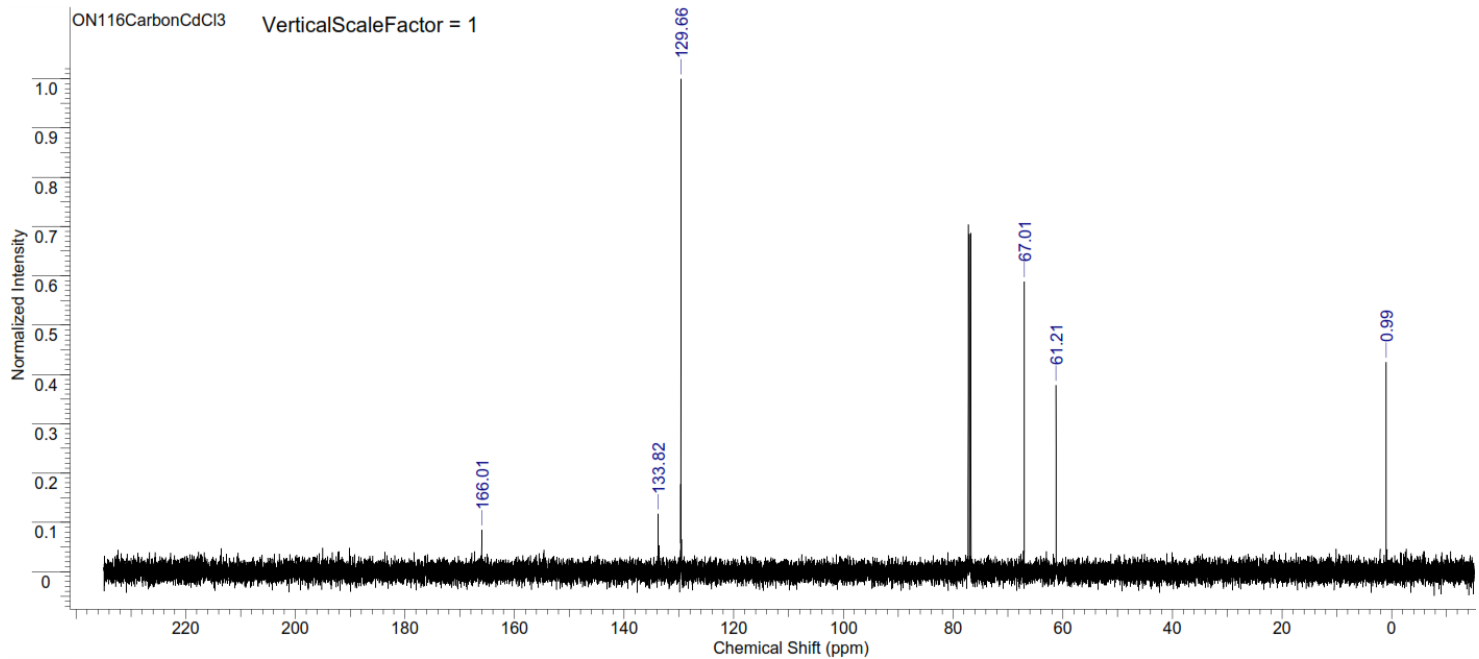

Figure S80. ${ }^{13} \mathrm{C}$ NMR spectrum of polyethylene terephthalate silicon acetal (Table S1, entry 15). 


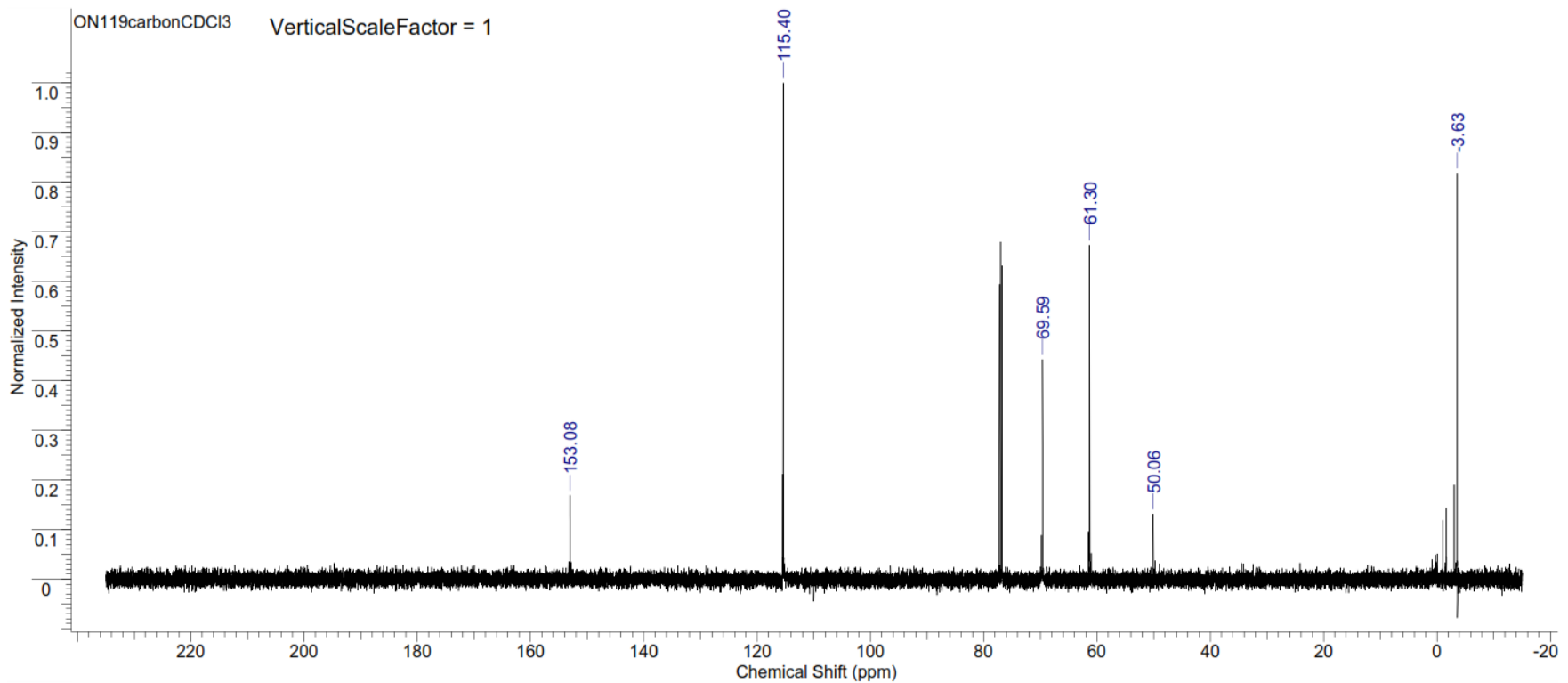

Figure S81. 13C NMR spectrum of 1,4-bis(2-((methoxydimethylsilyl)oxy)ethoxy)benzene (bis-silicon acetal monomer).

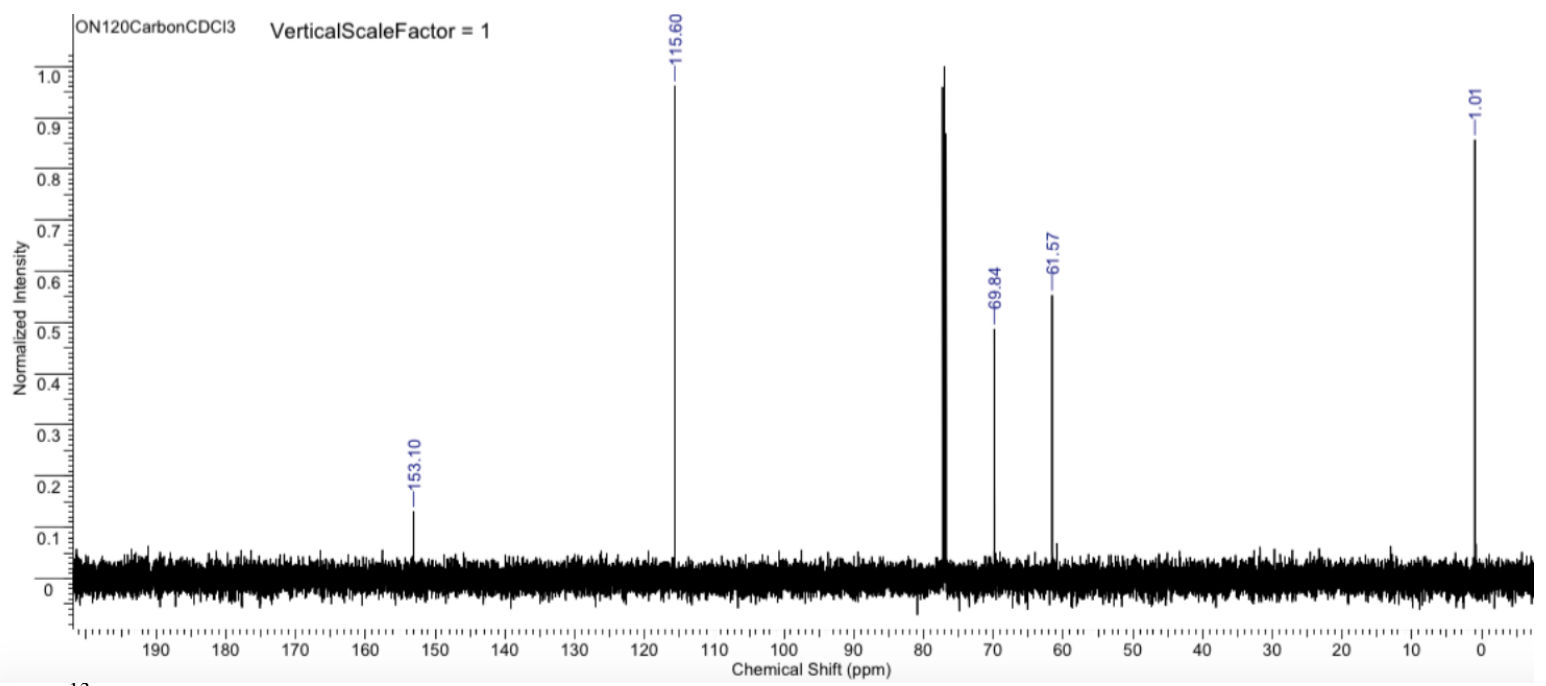

Figure S82. ${ }^{13} \mathrm{C}$ NMR spectrum of polyethylene hydroquinonoate silicon acetal (Table S1, entry 16). 


\section{Kinetic Studies of Silicon Acetal Metathesis}

\section{Dimethoxydimethylsilane : Diethoxydimethylsilane 1:1 ratio}

In an NMR tube, $0.30 \mathrm{~mL}(2.19 \mathrm{mmol})$ of dimethoxydimethylsilane was mixed with $0.37 \mathrm{~mL}$ (2.19 mmol) of diethoxydimethylsilane. A small crystal of $p$-TSA (less than $3 \mathrm{mg}$ ) and $1 \mathrm{~mL}$ of deuterated benzene (benzene- $d_{6}$ ) were added. An arrayed NMR experiment was quickly started using a $500 \mathrm{MHz}$ NMR instrument where 200 scans were taken every 3 minutes.
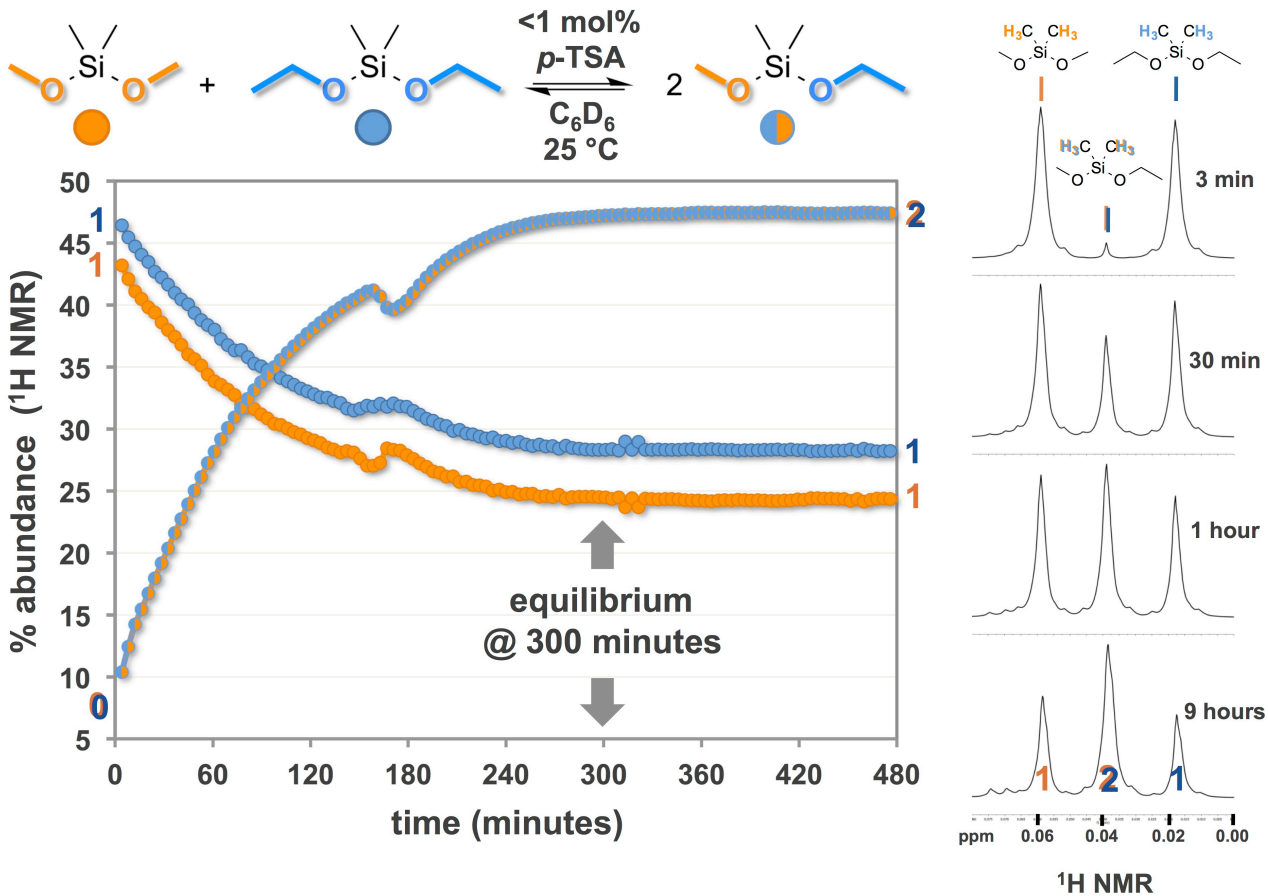

Figure S83. Acid-catalyzed ( $p$-TSA, $<1 \mathrm{~mol} \%$ ) silicon acetal metathesis converts a 1:1 ratio of symmetrical silicon acetals to yield the equilibrium amount of unsymmetrical silicon acetal after approximately 300 minutes at room temperature in benzene.

\begin{tabular}{|c|c|c|c|c|c|c|c|c|}
\hline Exp. & $\begin{array}{l}\text { Intensity of } \\
\text { dimethoxy }\end{array}$ & $\begin{array}{l}\text { Intensity of } \\
\text { mixed }\end{array}$ & $\begin{array}{c}\text { Intensity of } \\
\text { diethoxy }\end{array}$ & $\begin{array}{c}\text { Sum of } \\
\text { intensities }\end{array}$ & $\begin{array}{l}\% \text { Abundance of } \\
\text { dimethoxy }\end{array}$ & $\begin{array}{l}\% \text { Abundance } \\
\text { of mixed }\end{array}$ & $\begin{array}{l}\% \text { Abundance } \\
\text { of diethoxy }\end{array}$ & $\begin{array}{l}\text { Time } \\
(\mathrm{min})\end{array}$ \\
\hline 1 & 50.62 & 12.18 & 54.41 & 117.21 & 43.18744134 & 10.39160481 & 46.42095384 & 4 \\
\hline 2 & 50.22 & 14.84 & 54.23 & 119.29 & 42.09908626 & 12.44027161 & 45.46064213 & 8 \\
\hline 3 & 50.39 & 17.44 & 54.84 & 122.67 & 41.07768811 & 14.21700497 & 44.70530692 & 12 \\
\hline 4 & 50.11 & 19.13 & 54.54 & 123.78 & 40.4831152 & 15.45483923 & 44.06204556 & 16 \\
\hline 5 & 49.11 & 20.61 & 53.6 & 123.32 & 39.82322413 & 16.71261758 & 43.46415829 & 20 \\
\hline 6 & 46.31 & 21.12 & 50.18 & 117.61 & 39.37590341 & 17.95765666 & 42.66643993 & 24 \\
\hline 7 & 46.35 & 23.01 & 50.7 & 120.06 & 38.60569715 & 19.16541729 & 42.22888556 & 28 \\
\hline 8 & 46.07 & 24.7 & 50.52 & 121.29 & 37.9833457 & 20.36441586 & 41.65223844 & 32 \\
\hline 9 & 45.02 & 25.98 & 49.3 & 120.3 & 37.42310889 & 21.59600998 & 40.98088113 & 36 \\
\hline 10 & 44.64 & 27.59 & 49.07 & 121.3 & 36.80131904 & 22.74525969 & 40.45342127 & 40 \\
\hline 11 & 46.1 & 30.65 & 51.3 & 128.05 & 36.00156189 & 23.93596251 & 40.0624756 & 44 \\
\hline 12 & 43.35 & 30.41 & 47.85 & 121.61 & 35.64673958 & 25.00616726 & 39.34709317 & 48 \\
\hline 13 & 42.37 & 31.53 & 46.78 & 120.68 & 35.10938018 & 26.1269473 & 38.76367252 & 52 \\
\hline 14 & 42.93 & 34 & 47.92 & 124.85 & 34.38526231 & 27.23267922 & 38.38205847 & 56 \\
\hline 15 & 42.91 & 35.73 & 48.21 & 126.85 & 33.82735514 & 28.16712653 & 38.00551833 & 60 \\
\hline 16 & 40.16 & 34.91 & 44.58 & 119.65 & 33.56456331 & 29.17676557 & 37.25867112 & 64 \\
\hline 17 & 38.92 & 35.32 & 43.14 & 117.38 & 33.157267 & 30.09030499 & 36.75242801 & 68 \\
\hline 18 & 38.16 & 36.08 & 42.35 & 116.59 & 32.73007977 & 30.94605026 & 36.32386997 & 72 \\
\hline 19 & 40.24 & 39.85 & 45.74 & 125.83 & 31.97965509 & 31.6697131 & 36.3506318 & 76 \\
\hline 20 & 38.07 & 38.85 & 42.85 & 119.77 & 31.78592302 & 32.43717124 & 35.77690574 & 80 \\
\hline 21 & 36.62 & 38.39 & 40.88 & 115.89 & 31.59893002 & 33.1262404 & 35.27482958 & 84 \\
\hline 22 & 36.66 & 39.71 & 41.22 & 117.59 & 31.17612042 & 33.76987839 & 35.05400119 & 88 \\
\hline 23 & 36.34 & 40.49 & 40.93 & 117.76 & 30.859375 & 34.38349185 & 34.75713315 & 92 \\
\hline 24 & 36.98 & 42.54 & 42.04 & 121.56 & 30.42119118 & 34.99506417 & 34.58374465 & 96 \\
\hline 25 & 35.58 & 41.74 & 40 & 117.32 & 30.32730992 & 35.57790658 & 34.0947835 & 100 \\
\hline 26 & 35.46 & 42.67 & 39.95 & 118.08 & 30.0304878 & 36.13651762 & 33.83299458 & 104 \\
\hline
\end{tabular}




\begin{tabular}{|c|c|c|c|c|c|c|c|c|}
\hline 27 & 35.16 & 43.33 & 39.66 & 118.15 & 29.75878121 & 36.67371985 & 33.56749894 & 108 \\
\hline 28 & 34.48 & 43.34 & 38.78 & 116.6 & 29.57118353 & 37.16981132 & 33.25900515 & 112 \\
\hline 29 & 34.59 & 44.52 & 39.06 & 118.17 & 29.27138868 & 37.67453668 & 33.05407464 & 116 \\
\hline 30 & 34.12 & 44.77 & 38.51 & 117.4 & 29.06303237 & 38.13458262 & 32.80238501 & 120 \\
\hline 31 & 33.86 & 45.24 & 38.19 & 117.29 & 28.86861625 & 38.57106318 & 32.56032057 & 124 \\
\hline 32 & 34.4 & 47.04 & 39.24 & 120.68 & 28.50513755 & 38.97911833 & 32.51574412 & 128 \\
\hline 33 & 33.91 & 47.16 & 38.61 & 119.68 & 28.33389037 & 39.40508021 & 32.26102941 & 132 \\
\hline 34 & 33.85 & 47.9 & 38.65 & 120.4 & 28.11461794 & 39.78405316 & 32.1013289 & 136 \\
\hline 35 & 31.96 & 45.47 & 35.82 & 113.25 & 28.22075055 & 40.15011038 & 31.62913907 & 140 \\
\hline 36 & 31.86 & 45.84 & 35.71 & 113.41 & 28.09276078 & 40.41971607 & 31.48752315 & 144 \\
\hline 37 & 33.54 & 49.51 & 38.45 & 121.5 & 27.60493827 & 40.74897119 & 31.64609053 & 148 \\
\hline 38 & 35.15 & 53.44 & 41.48 & 130.07 & 27.0239102 & 41.08556931 & 31.89052049 & 152 \\
\hline 39 & 34.91 & 53.14 & 41.03 & 129.08 & 27.04524326 & 41.16826774 & 31.786489 & 156 \\
\hline 40 & 35.4 & 52.81 & 41.55 & 129.76 & 27.2811344 & 40.69821208 & 32.02065351 & 160 \\
\hline 41 & 33.29 & 46.59 & 37.23 & 117.11 & 28.4262659 & 39.7831099 & 31.7906242 & 164 \\
\hline 42 & 34.25 & 47.91 & 38.76 & 120.92 & 28.32451207 & 39.62123718 & 32.05425074 & 168 \\
\hline 43 & 33.31 & 47.06 & 37.49 & 117.86 & 28.26234516 & 39.928729 & 31.80892584 & 172 \\
\hline 44 & 33.47 & 48.39 & 38.14 & 120 & 27.89166667 & 40.325 & 31.78333333 & 176 \\
\hline 45 & 32.83 & 48.77 & 37.47 & 119.07 & 27.57201646 & 40.95909969 & 31.46888385 & 180 \\
\hline 46 & 32.24 & 49.28 & 36.89 & 118.41 & 27.22743012 & 41.61810658 & 31.15446331 & 184 \\
\hline 47 & 31.79 & 49.81 & 36.37 & 117.97 & 26.94752903 & 42.22259897 & 30.829872 & 188 \\
\hline 48 & 31.92 & 51.33 & 36.83 & 120.08 & 26.58227848 & 42.74650233 & 30.67121919 & 192 \\
\hline 49 & 31.03 & $\begin{array}{l}50.7 \\
\end{array}$ & 35.62 & 117.35 & 26.44226672 & 43.20409033 & 30.35364295 & 196 \\
\hline 50 & 30.89 & 51.51 & 35.69 & 118.09 & 26.15801507 & 43.61927344 & 30.22271149 & 200 \\
\hline 51 & 29.97 & 50.37 & 34.15 & 114.49 & 26.17695869 & 43.99510874 & 29.82793257 & 204 \\
\hline 52 & 30.51 & 52.57 & 35.5 & 118.58 & 25.72946534 & 44.33293979 & 29.93759487 & 208 \\
\hline 53 & 29.75 & 51.59 & 34.24 & 115.58 & 25.73974736 & 44.63575013 & 29.62450251 & 212 \\
\hline 54 & 29.9 & 52.7 & 34.69 & 117.29 & 25.49236934 & 44.9313667 & 29.57626396 & 216 \\
\hline 55 & 29.49 & 52.39 & 34.06 & 115.94 & 25.43557012 & 45.18716578 & 29.3772641 & 220 \\
\hline 56 & 29.33 & 52.57 & 33.81 & 115.71 & 25.34785239 & 45.43254688 & 29.21960073 & 224 \\
\hline 57 & 29.71 & 54.22 & 34.81 & 118.74 & 25.0210544 & 45.66279266 & 29.31615294 & 228 \\
\hline 58 & 28.99 & 52.94 & 33.49 & 115.42 & 25.11696413 & 45.86726737 & 29.0157685 & 232 \\
\hline 59 & 29.32 & 54.21 & 34.2 & 117.73 & 24.90444237 & 46.04603754 & 29.04952009 & 236 \\
\hline 60 & 28.77 & 53.33 & 33.31 & 115.41 & 24.92851573 & 46.20916732 & 28.86231696 & 240 \\
\hline 61 & 29.34 & 55.04 & 34.39 & 118.77 & 24.70320788 & 46.34166877 & 28.95512335 & 244 \\
\hline 62 & 28.9 & 54.21 & 33.53 & 116.64 & 24.77709191 & 46.47633745 & 28.74657064 & 248 \\
\hline 63 & 28.39 & 53.39 & 32.77 & 114.55 & 24.78393715 & 46.60846792 & 28.60759494 & 252 \\
\hline 64 & 29.07 & 55.34 & 34.06 & 118.47 & 24.53785769 & 46.71224783 & 28.74989449 & 256 \\
\hline 65 & 28.75 & 54.69 & 33.41 & 116.85 & 24.60419341 & 46.80359435 & 28.59221224 & 260 \\
\hline 66 & 29.16 & 55.8 & 34.08 & 119.04 & 24.49596774 & 46.875 & 28.62903226 & 264 \\
\hline 67 & 28.62 & 54.44 & 32.94 & 116 & 24.67241379 & 46.93103448 & 28.39655172 & 268 \\
\hline 68 & 29.46 & 56.8 & 34.63 & 120.89 & 24.36926131 & 46.98486227 & 28.64587642 & 272 \\
\hline 69 & 29.2 & 56.03 & 33.93 & 119.16 & 24.50486741 & 47.02081235 & 28.47432024 & 276 \\
\hline 70 & 28.96 & 55.55 & 33.52 & 118.03 & 24.53613488 & 47.06430568 & 28.39955943 & 280 \\
\hline 71 & 28.88 & 55.45 & 33.37 & 117.7 & 24.53695837 & 47.11129992 & 28.35174172 & 284 \\
\hline 72 & 28.91 & 55.54 & 33.36 & 117.81 & 24.53951277 & 47.14370597 & 28.31678126 & 288 \\
\hline 73 & 28.92 & 55.71 & 33.44 & 118.07 & 24.49394427 & 47.18387397 & 28.32218176 & 292 \\
\hline 74 & 29.09 & 56.09 & 33.62 & 118.8 & 24.48653199 & 47.21380471 & 28.2996633 & 296 \\
\hline 75 & 29.37 & 56.97 & 34.23 & 120.57 & 24.35929336 & 47.25055984 & 28.3901468 & 300 \\
\hline 76 & 29 & 55.99 & 33.47 & 118.46 & 24.48083741 & 47.26489954 & 28.25426304 & 304 \\
\hline 77 & 31.63 & 63.15 & 38.71 & 133.49 & 23.69465878 & 47.30691438 & 28.99842685 & 308 \\
\hline 78 & 29.12 & 56.4 & 33.76 & 119.28 & 24.41314554 & 47.28370221 & 28.30315225 & 312 \\
\hline 79 & 31.14 & 62.24 & 38.08 & 131.46 & 23.68781378 & 47.34520006 & 28.96698616 & 316 \\
\hline 80 & 28.96 & 56.23 & 33.64 & 118.83 & 24.3709501 & 47.31970041 & 28.30934949 & 320 \\
\hline 81 & 28.95 & 56.26 & 33.64 & 118.85 & 24.358435 & 47.33697939 & 28.30458561 & 324 \\
\hline 82 & 28.97 & 56.38 & 33.74 & 119.09 & 24.32613989 & 47.34234612 & 28.33151398 & 328 \\
\hline 83 & 28.99 & 56.39 & 33.71 & 119.09 & 24.34293392 & 47.35074314 & 28.30632295 & 332 \\
\hline 84 & 28.86 & 56.11 & 33.56 & 118.53 & 24.34826626 & 47.33822661 & 28.31350713 & 336 \\
\hline 85 & 29.03 & 56.52 & 33.81 & 119.36 & 24.3213807 & 47.35254692 & 28.32607239 & 340 \\
\hline 86 & 29.09 & 56.85 & 34.02 & 119.96 & 24.24974992 & 47.39079693 & 28.35945315 & 344 \\
\hline 87 & 28.86 & 56.52 & 33.81 & 119.19 & 24.21344072 & 47.42008558 & 28.3664737 & 348 \\
\hline 88 & 28.79 & 56.43 & 33.67 & 118.89 & 24.21566154 & 47.46404239 & 28.32029607 & 352 \\
\hline 89 & 28.88 & 56.66 & 33.85 & 119.39 & 24.18963062 & 47.45791105 & 28.35245833 & 356 \\
\hline 90 & 29.05 & 57.08 & 34.13 & 120.26 & 24.15599534 & 47.46382837 & 28.38017628 & 360 \\
\hline
\end{tabular}




\begin{tabular}{|c|c|c|c|c|c|c|c|c|}
\hline 91 & 28.85 & 56.53 & 33.77 & 119.15 & 24.21317667 & 47.44439782 & 28.34242551 & 364 \\
\hline 92 & 28.75 & 56.27 & 33.56 & 118.58 & 24.24523528 & 47.45319615 & 28.30156856 & 368 \\
\hline 93 & 28.69 & 56.2 & 33.54 & 118.43 & 24.22528076 & 47.45419235 & 28.32052689 & 372 \\
\hline 94 & 28.46 & 55.64 & 33.14 & 117.24 & 24.27499147 & 47.45820539 & 28.26680314 & 376 \\
\hline 95 & 28.45 & 55.62 & 33.17 & 117.24 & 24.26646196 & 47.44114637 & 28.29239168 & 380 \\
\hline 96 & 28.03 & 54.9 & 32.7 & 115.63 & 24.2411139 & 47.47902793 & 28.27985817 & 384 \\
\hline 97 & 28.04 & 54.81 & 32.67 & 115.52 & 24.27285319 & 47.44632964 & 28.28081717 & 388 \\
\hline 98 & 28.11 & 55.12 & 32.86 & 116.09 & 24.21397192 & 47.48040314 & 28.30562495 & 392 \\
\hline 99 & 28.21 & 55.34 & 33.02 & 116.57 & 24.20005147 & 47.473621 & 28.32632753 & 396 \\
\hline 100 & 28.17 & 55.3 & 33 & 116.47 & 24.18648579 & 47.48003778 & 28.33347643 & 400 \\
\hline 101 & 28.18 & 55.19 & 32.89 & 116.26 & 24.23877516 & 47.47118527 & 28.29003957 & 404 \\
\hline 102 & 28.36 & 55.54 & 33.17 & 117.07 & 24.22482276 & 47.44170155 & 28.3334757 & 408 \\
\hline 103 & 28.24 & 55.07 & 32.86 & 116.17 & 24.30920203 & 47.40466558 & 28.28613239 & 412 \\
\hline 104 & 28.15 & 54.9 & 32.78 & 115.83 & 24.30285764 & 47.3970474 & 28.30009497 & 416 \\
\hline 105 & 27.78 & 53.96 & 32.09 & 113.83 & 24.4048142 & 47.40402354 & 28.19116226 & 420 \\
\hline 106 & 27.81 & 53.99 & 32.16 & 113.96 & 24.4032994 & 47.37627238 & 28.22042822 & 424 \\
\hline 107 & 27.7 & 53.83 & 32.06 & 113.59 & 24.38594947 & 47.38973501 & 28.22431552 & 428 \\
\hline 108 & 27.77 & 53.93 & 32.14 & 113.84 & 24.39388616 & 47.37350668 & 28.23260717 & 432 \\
\hline 109 & 27.68 & 53.9 & 32.13 & 113.71 & 24.34262598 & 47.40128397 & 28.25609005 & 436 \\
\hline 110 & 27.67 & 53.9 & 32.12 & 113.69 & 24.33811241 & 47.40962266 & 28.25226493 & 440 \\
\hline 111 & 27.76 & 54.43 & 32.53 & 114.72 & 24.19804742 & 47.44595537 & 28.35599721 & 444 \\
\hline 112 & 27.29 & 53.19 & 31.63 & 112.11 & 24.34216395 & 47.44447418 & 28.21336188 & 448 \\
\hline 113 & 27.91 & 54.89 & 32.87 & 115.67 & 24.12898764 & 47.45396386 & 28.4170485 & 452 \\
\hline 114 & 27.55 & 53.85 & 32.08 & 113.48 & 24.27740571 & 47.45329573 & 28.26929855 & 456 \\
\hline 115 & 27.34 & 53.2 & 31.63 & 112.17 & 24.37371846 & 47.42801105 & 28.19827048 & 460 \\
\hline 116 & 27.32 & 53.15 & 31.63 & 112.1 & 24.37109723 & 47.41302409 & 28.21587868 & 464 \\
\hline 117 & 27.56 & 53.62 & 31.94 & 113.12 & 24.36350778 & 47.4009901 & 28.23550212 & 468 \\
\hline 118 & 27.59 & 53.73 & 32.06 & 113.38 & 24.33409772 & 47.38931028 & 28.27659199 & 472 \\
\hline
\end{tabular}

Dimethoxydimethylsilane : Diethoxydimethylsilane 1:2 ratio

In an NMR tube, $0.29 \mathrm{~mL}(2.08 \mathrm{mmol})$ of dimethoxydimethylsilane was mixed with $0.71 \mathrm{~mL}$ (4.16 mmol) of diethoxydimethylsilane. A small crystal of $p$-TSA (less than $2 \mathrm{mg}$ ) and $1.2 \mathrm{~mL}$ of deuterated benzene (benzene- $d_{6}$ ) were added. An arrayed NMR experiment was quickly started using a $500 \mathrm{MHz}$ NMR instrument where 120 scans were taken every 4 minutes.
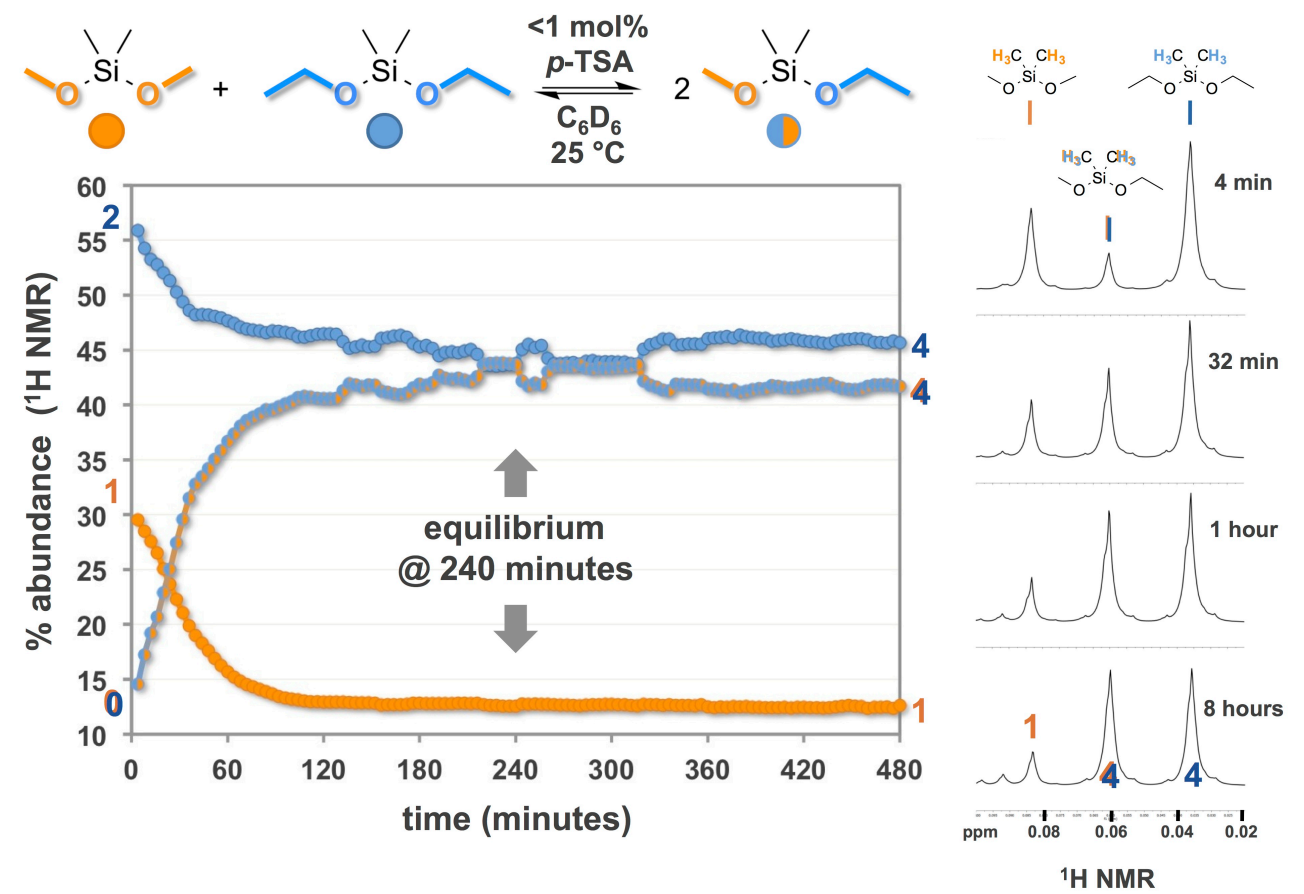

Figure S84. Acid-catalyzed ( $p$-TSA, $<1 \mathrm{~mol} \%$ ) silicon acetal metathesis converts a 1:2 ratio of symmetrical silicon acetals to yield the equilibrium amount of unsymmetrical silicon acetal after approximately 240 minutes at room temperature in benzene. 


\begin{tabular}{|c|c|c|c|c|c|c|c|c|}
\hline Exp. & $\begin{array}{l}\text { Intensity of } \\
\text { dimethoxy }\end{array}$ & $\begin{array}{c}\text { Intensity of } \\
\text { mixed }\end{array}$ & $\begin{array}{c}\text { Intensity of } \\
\text { diethoxy }\end{array}$ & $\begin{array}{c}\text { Sum of } \\
\text { intensities }\end{array}$ & $\begin{array}{l}\% \text { Abundance of } \\
\text { dimethoxy }\end{array}$ & $\begin{array}{l}\% \text { Abundance } \\
\text { of mixed }\end{array}$ & $\begin{array}{l}\% \text { Abundance } \\
\text { of diethoxy }\end{array}$ & $\begin{array}{l}\text { Time } \\
(\mathrm{min})\end{array}$ \\
\hline 1 & 15.84 & 7.81 & 29.96 & 53.61 & 29.54672636 & 14.56817758 & 55.88509606 & 4 \\
\hline 2 & 16.43 & 9.93 & 31.29 & 57.65 & 28.49956635 & 17.2246314 & 54.27580225 & 8 \\
\hline 3 & 16.59 & 11.55 & 32.05 & 60.19 & 27.56271806 & 19.18923409 & 53.24804785 & 12 \\
\hline 4 & 16.14 & 12.59 & 32.13 & 60.86 & 26.5198817 & 20.68682221 & 52.79329609 & 16 \\
\hline 5 & 15.27 & 13.96 & 31.72 & 60.95 & 25.0533224 & 22.90401969 & 52.04265792 & 20 \\
\hline 6 & 14.17 & 14.98 & 30.72 & 59.87 & 23.66794722 & 25.02087857 & 51.31117421 & 24 \\
\hline 7 & 13.18 & 16.21 & 29.71 & 59.1 & 22.30118443 & 27.42808799 & 50.27072758 & 28 \\
\hline 8 & 12.42 & 17.44 & 29.14 & 59 & 21.05084746 & 29.55932203 & 49.38983051 & 32 \\
\hline 9 & 11.6 & 18.37 & 28.34 & 58.31 & 19.89367175 & 31.50403018 & 48.60229806 & 36 \\
\hline 10 & 11.02 & 19.01 & 27.96 & 57.99 & 19.00327643 & 32.78151405 & 48.21520952 & 40 \\
\hline 11 & 10.41 & 19.07 & 27.48 & 56.96 & 18.27598315 & 33.47963483 & 48.24438202 & 44 \\
\hline 12 & 9.76 & 18.97 & 26.72 & 55.45 & 17.60144274 & 34.2110009 & 48.18755636 & 48 \\
\hline 13 & 9.23 & 19.15 & 26.27 & 54.65 & 16.88929552 & 35.04117109 & 48.06953339 & 52 \\
\hline 14 & 8.7 & 19.2 & 25.68 & 53.58 & 16.23740202 & 35.83426652 & 47.92833147 & 56 \\
\hline 15 & 8.21 & 19.2 & 24.95 & 52.36 & 15.67990833 & 36.66921314 & 47.65087853 & 60 \\
\hline 16 & 7.85 & 19.29 & 24.49 & 51.63 & 15.20433856 & 37.36199884 & 47.4336626 & 64 \\
\hline 17 & 7.71 & 19.79 & 24.46 & 51.96 & 14.83833718 & 38.08698999 & 47.07467283 & 68 \\
\hline 18 & 7.6 & 20.2 & 24.56 & 52.36 & 14.51489687 & 38.57906799 & 46.90603514 & 72 \\
\hline 19 & 7.52 & 20.43 & 24.6 & 52.55 & 14.31018078 & 38.87725975 & 46.81255947 & 76 \\
\hline 20 & 7.39 & 20.58 & 24.54 & 52.51 & 14.07350981 & 39.19253476 & 46.73395544 & 80 \\
\hline 21 & 7.35 & 20.93 & 24.64 & 52.92 & 13.88888889 & 39.55026455 & 46.56084656 & 84 \\
\hline 22 & 7.13 & 20.61 & 24.35 & 52.09 & 13.68784796 & 39.56613553 & 46.74601651 & 88 \\
\hline 23 & 6.75 & 19.99 & 23.44 & 50.18 & 13.45157433 & 39.83658828 & 46.71183739 & 92 \\
\hline 24 & 6.55 & 19.75 & 22.95 & 49.25 & 13.29949239 & 40.10152284 & 46.59898477 & 96 \\
\hline 25 & 6.44 & 19.68 & 22.7 & 48.82 & 13.19131503 & 40.31134781 & 46.49733716 & 100 \\
\hline 26 & 6.31 & 19.54 & 22.2 & 48.05 & 13.13215401 & 40.66597294 & 46.20187305 & 104 \\
\hline 27 & 6.24 & 19.51 & 22.1 & 47.85 & 13.04075235 & 40.77324974 & 46.18599791 & 108 \\
\hline 28 & 6.19 & 19.42 & 22.1 & 47.71 & 12.97421924 & 40.70425487 & 46.32152589 & 112 \\
\hline 29 & 6.26 & 19.63 & 22.44 & 48.33 & 12.95261742 & 40.61659425 & 46.43078833 & 116 \\
\hline 30 & 6.29 & 19.75 & 22.61 & 48.65 & 12.9290853 & 40.59609455 & 46.47482014 & 120 \\
\hline 31 & 6.36 & 19.95 & 22.85 & 49.16 & 12.93734744 & 40.5817738 & 46.48087876 & 124 \\
\hline 32 & 6.38 & 20.05 & 22.92 & 49.35 & 12.92806484 & 40.62816616 & 46.443769 & 128 \\
\hline 33 & 6.28 & 20.02 & 22.2 & 48.5 & 12.94845361 & 41.27835052 & 45.77319588 & 132 \\
\hline 34 & 6.21 & 20.18 & 21.72 & 48.11 & 12.90791935 & 41.94554147 & 45.14653918 & 136 \\
\hline 35 & 6.14 & 19.94 & 21.6 & 47.68 & 12.87751678 & 41.8204698 & 45.30201342 & 140 \\
\hline 36 & 6.07 & 19.66 & 21.45 & 47.18 & 12.86562103 & 41.67019924 & 45.46417974 & 144 \\
\hline 37 & 6.02 & 19.59 & 21.2 & 46.81 & 12.86049989 & 41.85003204 & 45.28946806 & 148 \\
\hline 38 & 6 & 19.55 & 21.21 & 46.76 & 12.8314799 & 41.80923867 & 45.35928144 & 152 \\
\hline 39 & 5.83 & 19.01 & 21.22 & 46.06 & 12.65740339 & 41.27225358 & 46.07034303 & 156 \\
\hline 40 & 5.92 & 19.21 & 21.53 & 46.66 & 12.68752679 & 41.17016717 & 46.14230604 & 160 \\
\hline 41 & 5.98 & 19.33 & 21.81 & 47.12 & 12.6910017 & 41.0229202 & 46.2860781 & 164 \\
\hline 42 & 6.02 & 19.41 & 21.96 & 47.39 & 12.70310192 & 40.95800802 & 46.33889006 & 168 \\
\hline 43 & 6.03 & 19.44 & 21.84 & 47.31 & 12.74571972 & 41.0906785 & 46.16360178 & 172 \\
\hline 44 & 6.03 & 19.55 & 21.44 & 47.02 & 12.82433007 & 41.57805189 & 45.59761803 & 176 \\
\hline 45 & 6 & 19.62 & 21.2 & 46.82 & 12.81503631 & 41.90516873 & 45.27979496 & 180 \\
\hline 46 & 5.94 & 19.42 & 21.1 & 46.46 & 12.78519156 & 41.79939733 & 45.41541111 & 184 \\
\hline 47 & 5.93 & 19.47 & 20.9 & 46.3 & 12.80777538 & 42.05183585 & 45.14038877 & 188 \\
\hline 48 & 5.83 & 19.47 & 20.28 & 45.58 & 12.79069767 & 42.71610355 & 44.49319877 & 192 \\
\hline 49 & 5.89 & 19.55 & 20.62 & 46.06 & 12.78766826 & 42.44463743 & 44.76769431 & 196 \\
\hline 50 & 5.97 & 19.74 & 20.92 & 46.63 & 12.80291658 & 42.33326185 & 44.86382157 & 200 \\
\hline 51 & 6.01 & 19.89 & 20.98 & 46.88 & 12.81996587 & 42.4274744 & 44.75255973 & 204 \\
\hline 52 & 6.05 & 19.95 & 21.2 & 47.2 & 12.81779661 & 42.26694915 & 44.91525424 & 208 \\
\hline 53 & 6.1 & 20.07 & 21.45 & 47.62 & 12.80974381 & 42.14615708 & 45.04409912 & 212 \\
\hline 54 & 5.98 & 19.84 & 20.79 & 46.61 & 12.82986484 & 42.56597297 & 44.6041622 & 216 \\
\hline 55 & 5.58 & 19.19 & 19.14 & 43.91 & 12.70781143 & 43.70302892 & 43.58915964 & 220 \\
\hline 56 & 5.4 & 18.73 & 18.64 & 42.77 & 12.6256722 & 43.79237783 & 43.58194996 & 224 \\
\hline 57 & 5.36 & 18.63 & 18.5 & 42.49 & 12.61473288 & 43.84561073 & 43.53965639 & 228 \\
\hline 58 & 5.35 & 18.65 & 18.54 & 42.54 & 12.57639868 & 43.84109074 & 43.58251058 & 232 \\
\hline 59 & 5.27 & 18.35 & 18.31 & 41.93 & 12.56856666 & 43.76341522 & 43.66801813 & 236 \\
\hline 60 & 5.29 & 18.38 & 18.41 & 42.08 & 12.57129278 & 43.67870722 & 43.75 & 240 \\
\hline 61 & 5.87 & 19.38 & 20.7 & 45.95 & 12.77475517 & 42.17627856 & 45.04896627 & 244 \\
\hline
\end{tabular}




\begin{tabular}{|c|c|c|c|c|c|c|c|c|}
\hline 62 & 5.9 & 19.32 & 21.09 & 46.31 & 12.74022889 & 41.71885122 & 45.54091989 & 248 \\
\hline 63 & 5.96 & 19.6 & 21.09 & 46.65 & 12.77599143 & 42.01500536 & 45.20900322 & 252 \\
\hline 64 & 6 & 19.71 & 21.4 & 47.11 & 12.73614944 & 41.8382509 & 45.42559966 & 256 \\
\hline 65 & 5.84 & 19.74 & 20.29 & 45.87 & 12.73163288 & 43.03466318 & 44.23370395 & 260 \\
\hline 66 & 5.6 & 19.24 & 19.32 & 44.16 & 12.68115942 & 43.56884058 & 43.75 & 264 \\
\hline 67 & 5.53 & 19.01 & 19.11 & 43.65 & 12.66895762 & 43.55097365 & 43.78006873 & 268 \\
\hline 68 & 5.52 & 18.98 & 19.12 & 43.62 & 12.65474553 & 43.51215039 & 43.83310408 & 272 \\
\hline 69 & 5.47 & 18.84 & 19 & 43.31 & 12.62987763 & 43.50034634 & 43.86977603 & 276 \\
\hline 70 & 5.36 & 18.57 & 18.6 & 42.53 & 12.60286856 & 43.6632965 & 43.73383494 & 280 \\
\hline 71 & 5.53 & 18.95 & 19.22 & $\begin{array}{ll}43.7 \\
\end{array}$ & 12.65446224 & 43.36384439 & 43.98169336 & 284 \\
\hline 72 & 5.67 & 19.31 & 19.66 & 44.64 & 12.7016129 & 43.25716846 & 44.04121864 & 288 \\
\hline 73 & 5.7 & 19.48 & 19.71 & 44.89 & 12.6977055 & 43.39496547 & 43.90732903 & 292 \\
\hline 74 & 5.75 & 19.56 & 19.84 & 45.15 & 12.73532669 & 43.32225914 & 43.94241417 & 296 \\
\hline 75 & 5.7 & 19.42 & 19.68 & $\begin{array}{lll}44.8 \\
\end{array}$ & 12.72321429 & 43.34821429 & 43.92857143 & 300 \\
\hline 76 & 5.61 & 19.19 & 19.4 & 44.2 & 12.69230769 & 43.41628959 & 43.89140271 & 304 \\
\hline 77 & 5.57 & 19.07 & 19.27 & 43.91 & 12.68503758 & 43.42974266 & 43.88521977 & 308 \\
\hline 78 & 5.4 & 18.65 & 18.72 & 42.77 & 12.6256722 & 43.60533084 & 43.76899696 & 312 \\
\hline 79 & 5.38 & 18.63 & 18.67 & 42.68 & 12.6054358 & 43.65042174 & 43.74414246 & 316 \\
\hline 80 & 5.79 & 19.17 & 20.49 & 45.45 & 12.73927393 & 42.17821782 & 45.08250825 & 320 \\
\hline 81 & 5.89 & 19.38 & 21.08 & 46.35 & 12.70765912 & 41.81229773 & 45.48004315 & 324 \\
\hline 82 & 5.92 & 19.42 & 21.3 & 46.64 & 12.69296741 & 41.6380789 & 45.66895369 & 328 \\
\hline 83 & 5.9 & 19.3 & 21.47 & 46.67 & 12.64195415 & 41.35418899 & 46.00385687 & 332 \\
\hline 84 & 5.91 & 19.23 & 21.42 & 46.56 & 12.69329897 & 41.30154639 & 46.00515464 & 336 \\
\hline 85 & 5.89 & 19.55 & 21.2 & 46.64 & 12.62864494 & 41.91680961 & 45.45454545 & 340 \\
\hline 86 & 5.88 & 19.52 & 21.21 & 46.61 & 12.6153186 & 41.87942502 & 45.50525638 & 344 \\
\hline 87 & 5.85 & 19.37 & 21.1 & 46.32 & 12.62953368 & 41.81778929 & 45.55267703 & 348 \\
\hline 88 & 5.8 & 19.27 & 20.98 & 46.05 & 12.59500543 & 41.84581976 & 45.55917481 & 352 \\
\hline 89 & 5.7 & 18.78 & 20.46 & 44.94 & 12.6835781 & 41.78905207 & 45.52736983 & 356 \\
\hline 90 & 5.54 & 18.36 & 20.39 & 44.29 & 12.50846692 & 41.45405283 & 46.03748024 & 360 \\
\hline 91 & 5.6 & 18.69 & 20.78 & 45.07 & 12.42511649 & 41.46882627 & 46.10605724 & 364 \\
\hline 92 & 5.65 & 18.74 & 20.89 & 45.28 & 12.47791519 & 41.3869258 & 46.13515901 & 368 \\
\hline 93 & 5.71 & 18.91 & 21.17 & 45.79 & 12.46997161 & 41.29722647 & 46.23280192 & 372 \\
\hline 94 & 5.69 & 18.8 & 20.94 & 45.43 & 12.52476337 & 41.38234647 & 46.09289016 & 376 \\
\hline 95 & 5.67 & 18.65 & 21.02 & 45.34 & 12.5055139 & 41.13365682 & 46.36082929 & 380 \\
\hline 96 & 5.62 & 18.53 & 20.75 & 44.9 & 12.51670379 & 41.26948775 & 46.21380846 & 384 \\
\hline 97 & 5.55 & 18.42 & 20.53 & $\begin{array}{l}44.5 \\
\end{array}$ & 12.47191011 & 41.39325843 & 46.13483146 & 388 \\
\hline 98 & 5.51 & 18.34 & 20.36 & 44.21 & 12.46324361 & 41.48382719 & 46.0529292 & 392 \\
\hline 99 & 5.52 & 18.41 & 20.45 & 44.38 & 12.43803515 & 41.48264984 & 46.07931501 & 396 \\
\hline 100 & 5.51 & 18.5 & 20.28 & 44.29 & 12.44073154 & 41.77015128 & 45.78911718 & 400 \\
\hline 101 & 5.64 & 18.94 & 20.83 & 45.41 & 12.42017177 & 41.7088747 & 45.87095353 & 404 \\
\hline 102 & 5.65 & 18.88 & 20.84 & 45.37 & 12.45316288 & 41.61340093 & 45.93343619 & 408 \\
\hline 103 & 5.68 & 19.02 & 21.08 & 45.78 & 12.4071647 & 41.54652687 & 46.04630843 & 412 \\
\hline 104 & 5.65 & 18.88 & 20.85 & 45.38 & 12.45041869 & 41.60423094 & 45.94535037 & 416 \\
\hline 105 & 5.57 & 18.68 & 20.51 & 44.76 & 12.44414656 & 41.7336908 & 45.82216265 & 420 \\
\hline 106 & 5.52 & 18.59 & 20.34 & 44.45 & 12.41844769 & 41.82227222 & 45.75928009 & 424 \\
\hline 107 & 5.45 & 18.36 & 20.07 & 43.88 & 12.42023701 & 41.8413856 & 45.73837739 & 428 \\
\hline 108 & 5.41 & 18.31 & 19.91 & 43.63 & 12.39972496 & 41.96653679 & 45.63373825 & 432 \\
\hline 109 & 5.43 & 18.33 & 19.92 & 43.68 & 12.43131868 & 41.96428571 & 45.6043956 & 436 \\
\hline 110 & 5.72 & 19.11 & 21.01 & 45.84 & 12.47818499 & 41.68848168 & 45.83333333 & 440 \\
\hline 111 & 5.9 & 19.52 & 21.59 & 47.01 & 12.55052117 & 41.5230802 & 45.92639864 & 444 \\
\hline 112 & 6.02 & 19.77 & 21.94 & 47.73 & 12.61261261 & 41.42049026 & 45.96689713 & 448 \\
\hline 113 & 6.1 & 20.1 & 22.36 & 48.56 & 12.56177924 & 41.39209226 & 46.0461285 & 452 \\
\hline 114 & 5.88 & 19.49 & 21.63 & 47 & 12.5106383 & 41.46808511 & 46.0212766 & 456 \\
\hline 115 & 5.7 & 19.21 & 21.17 & 46.08 & 12.36979167 & 41.68836806 & 45.94184028 & 460 \\
\hline 116 & 5.61 & 18.83 & 20.57 & 45.01 & 12.46389691 & 41.83514774 & 45.70095534 & 464 \\
\hline 117 & 5.59 & 18.77 & 20.5 & 44.86 & 12.46098975 & 41.84128399 & 45.69772626 & 468 \\
\hline 118 & 5.58 & 18.72 & 20.4 & 44.7 & 12.48322148 & 41.87919463 & 45.63758389 & 472 \\
\hline 119 & 5.62 & 19.01 & 20.84 & 45.47 & 12.35979767 & 41.80778535 & 45.83241698 & 476 \\
\hline 120 & 6.04 & 19.95 & 21.85 & 47.84 & 12.62541806 & 41.70150502 & 45.67307692 & 480 \\
\hline
\end{tabular}


In an NMR tube, $0.57 \mathrm{~mL}(4.16 \mathrm{mmol})$ of dimethoxydimethylsilane was mixed with $0.71 \mathrm{~mL}(4.16 \mathrm{mmol})$ of diethoxydimethylsilane and $1.2 \mathrm{~mL}$ of deuterated benzene (benzene- $d_{6}$ ) were added.

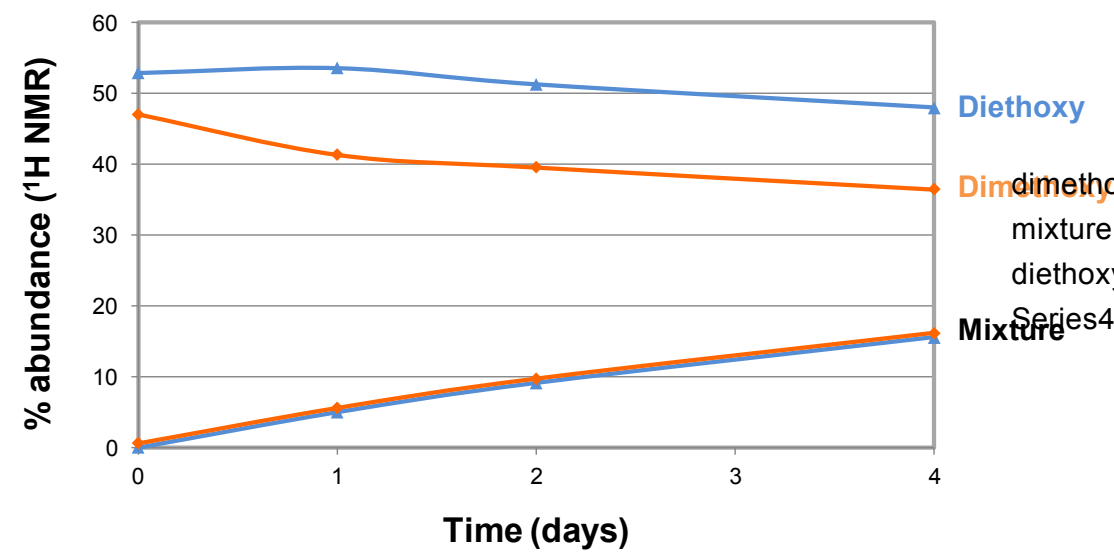

Figure S85. Without added catalyst, silicon acetal metathesis equilibrates symmetrical silicon acetals slowly to yield the unsymmetrical silicon acetal at room temperature in benzene. The exchange was much slower compared to the acidcatalyzed reaction, which reached equilibrium in 4 to 5 hours at room temperature. Without catalyst, only $15.5 \%$ of the mixed silicon acetal was obtained after 4 days at room temperature. This system is far from equilibrium since $50 \%$ of the mixed silicon acetal is expected at equilibrium.

\begin{tabular}{|c|c|c|c|c|c|c|c|c|}
\hline Exp. & $\begin{array}{c}\text { Intensity of } \\
\text { dimethoxy }\end{array}$ & $\begin{array}{c}\text { Intensity of } \\
\text { mixed }\end{array}$ & $\begin{array}{c}\text { Intensity of } \\
\text { diethoxy }\end{array}$ & $\begin{array}{c}\text { Sum of } \\
\text { intensities }\end{array}$ & $\begin{array}{c}\text { \% Abundance of } \\
\text { dimethoxy }\end{array}$ & $\begin{array}{c}\text { \% Abundance } \\
\text { of mixed }\end{array}$ & $\begin{array}{c}\text { \% Abundance } \\
\text { of diethoxy }\end{array}$ & $\begin{array}{c}\text { Time } \\
\text { (days) }\end{array}$ \\
\hline 1 & 10 & 0 & 11.25 & 21.25 & 47.05882 & 0 & 52.94118 & 0 \\
\hline 2 & 9.99 & 1.20 & 12.95 & 24.14 & 41.38340 & 4.971002 & 53.64560 & 1 \\
\hline 3 & 9.98 & 2.29 & 12.93 & 25.20 & 39.603175 & 9.0873016 & 51.309524 & 2 \\
\hline 4 & 10 & 4.25 & 13.14 & 27.39 & 36.509675 & $\mathbf{1 5 . 5 1 6 6 1 2}$ & 47.973713 & 4 \\
\hline
\end{tabular}


Proposed Mechanism for SAMP (silicon acetal metathesis polymerization)

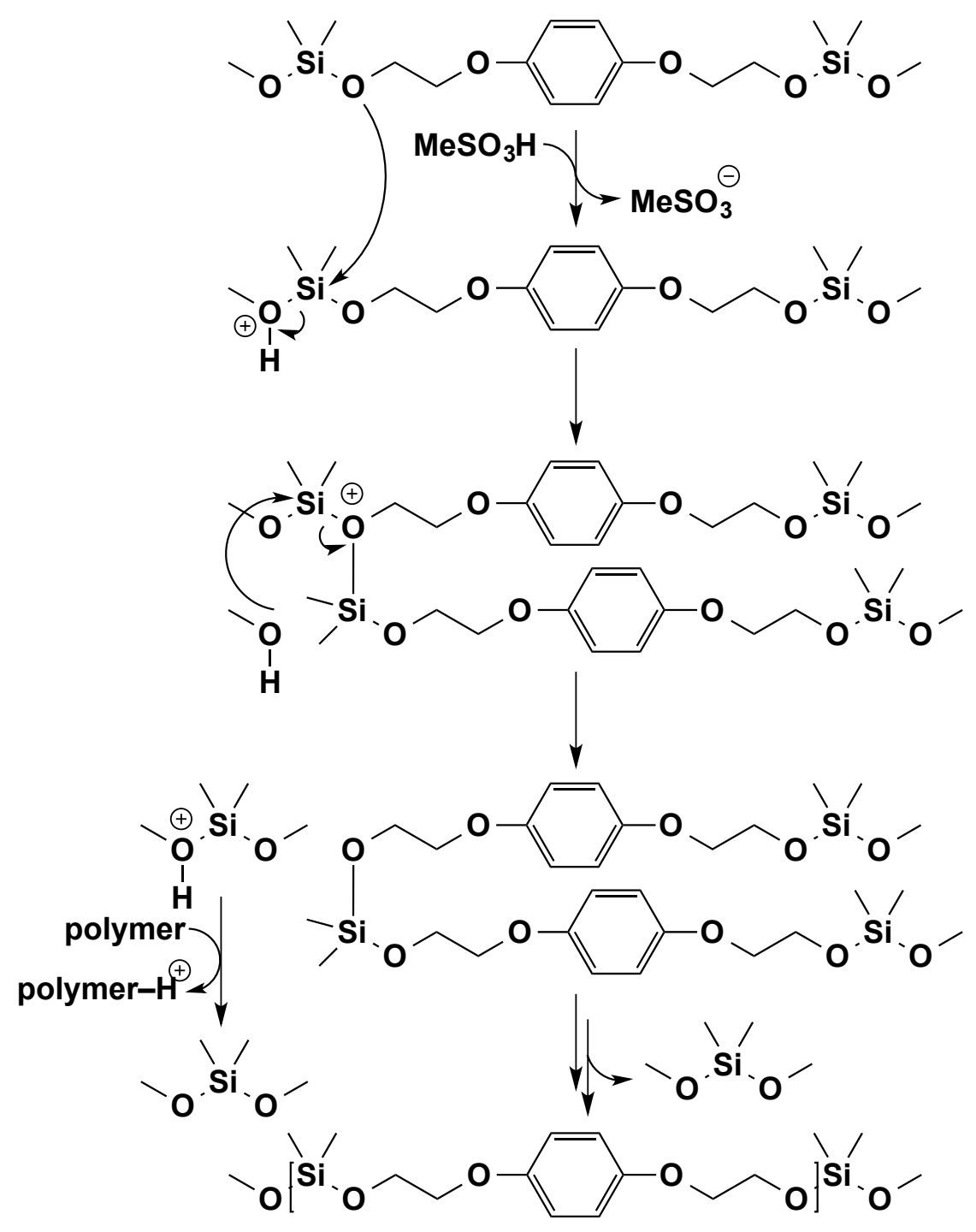

